\title{
Architecture-dependent interplay between self-assembly and crystallization in discrete block co-oligomers
}

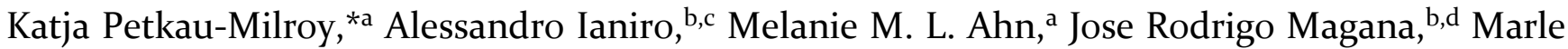
E. J. Vleugels, ${ }^{a}$ Brigitte A. G. Lamers, ${ }^{a}$ Remco Tuinier, ${ }^{b, c}$ Ilja K. Voets, ${ }^{\text {b,d }}$ Anja R. A. Palmans, ${ }^{* a, b}$ E.W. Meijer ${ }^{* a, b}$

${ }^{a}$ Laboratory of Macromolecular and Organic Chemistry, ${ }^{b}$ Institute for Complex Molecular Systems, 'Laboratory of Physical Chemistry, and ${ }^{\mathrm{d}}$ Laboratory of Self-Organizing Soft Matter, Eindhoven University of Technology, P.O. Box 513, 560o MB Eindhoven, The Netherlands

Corresponding authors: k.milroy@gmx.net; a.palmans@tue.nl; e.w.meijer@tue.nl.

\section{Table of Contents}

1. Materials and experimental methods 2

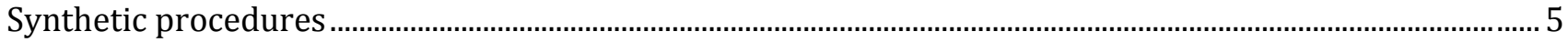

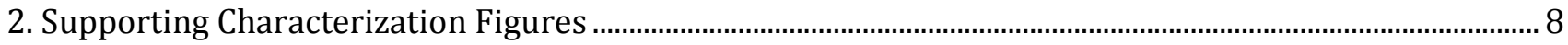

3. Degradation study of discrete lactic acid-based oligomers ........................................................................... 22

4. Stability study of alcohol-terminated and TBDMS-terminated BCOs........................................................... 23

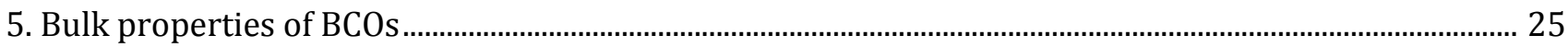

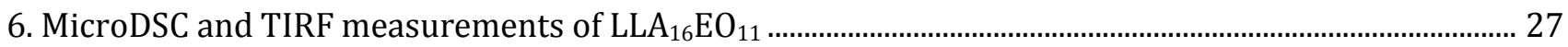

7. Scheutjens-Fleer Self-Consistent Field (SCF) computations......................................................................... 27

Setup of the numerical SCF lattice computations ................................................................................. 27

Modeling of the self-assembly of monodisperse LLA $_{16}-\mathrm{EO}_{\mathbf{n}}$ copolymers in water.......................... 28

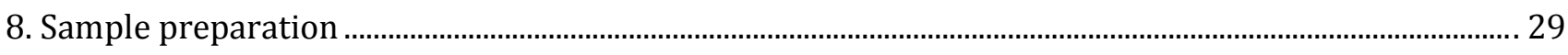

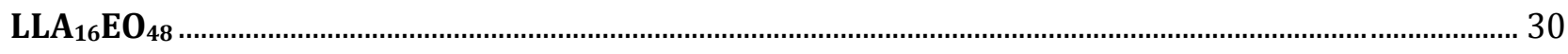

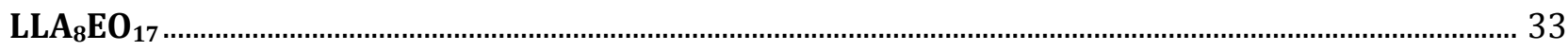

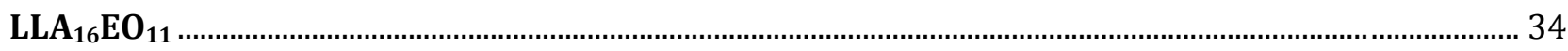

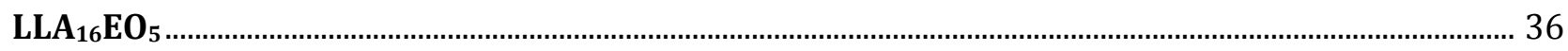

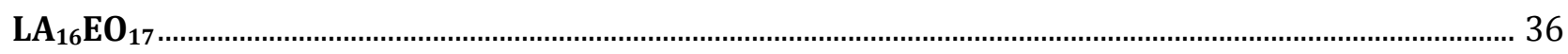

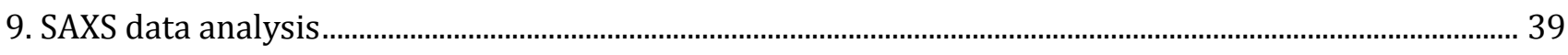

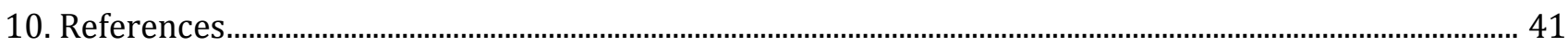




\section{Materials and experimental methods}

Most reagents were purchased from Aldrich and used as received. The monodisperse ethylene

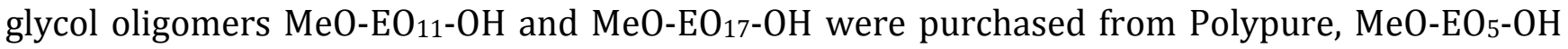

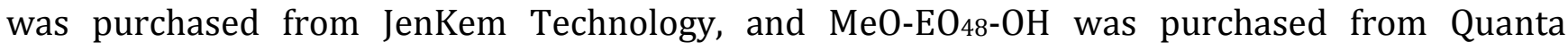
BioDesign. Prior to use, all ethylene glycol oligomers were dried in vacuo in an oven at $40^{\circ} \mathrm{C}$ over phosphorus pentoxide overnight. All solvents were purchased from Biosolve and dry solvents were obtained using MBraun solvent purification system (MB SPS-800). Deuterated compounds were obtained from Cambridge Isotopes Laboratories. Reactions were followed by thin-layer chromatography (TLC) using 60-F254 silica gel plates from Merck and visualized by cerium molybdate (CeMo) stain.

Automated column chromatography was performed on a Grace Reveleris X2 using Reveleris Silica Flash Cartridges.

${ }^{1} \mathrm{H}$ NMR and ${ }^{13} \mathrm{C}$ NMR spectra were recorded on a Varian Mercury Vx $400 \mathrm{MHz}$ (400 MHz for ${ }^{1} \mathrm{H}-$ NMR and $100 \mathrm{MHz}$ for ${ }^{13} \mathrm{C}$-NMR). Proton chemical shifts are reported in ppm ( $\delta$ ) downfield from tetramethylsilane (TMS) using the resonance frequency of the deuterated solvent as the internal standard. Peak multiplicities are abbreviated as s: singlet; d: doublet, q: quartet; p: pentet; m: multiplet; dd: double doublet; dt: double triplet; dq: double quartet; Carbon chemical shifts are reported in ppm $(\delta)$ downfield from TMS using the resonance frequency of the deuterated solvent as the internal standard.

Gas Chromatography-Mass Spectrometry (GC-MS) measurements were obtained on a Shimadzu GC-17A gas chromatograph with a Shimadzu AOC-20i auto injector, Shimadzu GCMSQP5000 mass spectrometer and a Phenomenex Zebron ZB-35 column $(l=30 \mathrm{~m}, \mathrm{ID}=0.25 \mathrm{~mm}$, film thickness $=0.25 \mu \mathrm{m}$ ).

Matrix assisted laser absorption/ionization mass time of flight (MALDI-TOF) spectra were obtained on a Bruker Autoflex Speed. $\alpha$-Cyano-4-hydroxycinnamic acid (CHCA) and trans-2-[3(4-tert-butylphenyl)-2-methyl-2-propenylidene]malononitrile (DCBT) were used as matrix. All samples were dissolved in chloroform.

Differential scanning calorimetry (DSC) data were collected on a DSC Q2000 TA Instruments, calibrated with indium as a standard. The samples were heated to $150^{\circ} \mathrm{C}\left(40^{\circ} \mathrm{C} / \mathrm{min}\right)$, cooled to $85^{\circ} \mathrm{C}\left(10^{\circ} \mathrm{C} / \mathrm{min}\right)$, and heated to $150{ }^{\circ} \mathrm{C}\left(10^{\circ} \mathrm{C} / \mathrm{min}\right)$. The last cooling and heating cycle were repeated to check for degradation. The peak maximum of the melting transition was used as melting point $\left(T_{\mathrm{m}}\right)$. The peak minimum of the crystallization transition was used as crystallization temperature $\left(T_{c}\right)$. The peak minimum of the glass transition temperatures $\left(T_{\mathrm{g}}\right)$ were taken at the mid-point of the transition.

Wide angle X-ray scattering (WAXS) experiments were performed on a Ganesha lab instrument (SAXSLAB) equipped with a GeniX-Cu ultra-low divergence X-ray generator, an evacuated flight tube and sample holder in a single housing, and a 2-dimensional Pilatus $300 \mathrm{~K}$ detector with $487 \times 619$ pixels of $172 \times 172 \mu \mathrm{m}$. The source produces X-rays with a wavelength $\lambda=$ $0.154 \mathrm{~nm}$ and a flux of $1 \times 10^{8} \mathrm{ph} \mathrm{s}^{-1}$. Two sample-to-detector distances of $84 \mathrm{~mm}$ and $431 \mathrm{~mm}$ were used to measure the scattering intensity as a function of the momentum transfer vector 
$q=4 \pi(\sin \theta) / \lambda$ (where $2 \theta$ is the scattering angle) to cover a $q$-range of $\mathrm{nm}^{-1}$. The beam centre and $q$-range of the instrument were calibrated with a silver behenate standard. The collected twodimensional WAXS patterns were brought to an absolute intensity scale using the calibrated detector response function, known sample-to-detector distances, and measured incident and transmitted beam intensities. Subsequently, the normalized WAXS patterns were azimuthally averaged to obtain the 1D WAXS profiles. Samples were prepared in a glass capillary with a diameter of $1 \mathrm{~mm}$, glass thickness $0.01 \mathrm{~mm}$ and length of $80 \mathrm{~mm}$. All samples were heated to 110 ${ }^{\circ} \mathrm{C}$ in vacuo, cooling down to RT occurred overnight as the oven cooled down to RT.

Solution SAXS: Synchrotron radiation X-ray scattering data was collected at the BM29 BioSAXS beamline of the European Synchrotron Radiation Facility (Grenoble, France) operating at 12.5 $\operatorname{keV}$ and $\lambda=0.992 \AA$. The beam size was set at about $700 \mu \mathrm{m} \times 700 \mu \mathrm{m}$, and two-dimensional scattering profiles were collected using a Pilatus $1 \mathrm{M}$ detector. Samples were measured at a fixed sample-to-detector distance of $2.867 \mathrm{~m}$ to cover an angular range of $0.025 \leq q \leq 5 \mathrm{~nm}^{-1}$. Samples were loaded via an automated sample changer and flowed through a quartz capillary of $1.8 \mathrm{~mm}$ in diameter, while collecting 10 frames of $0.1 \mathrm{~s}$ with a reduced flux of $10^{12}$ photons $\mathrm{s}^{-1}$. The averaged value of buffer scattering measured before and after the sample measurements was averaged and subtracted from the scattering of the samples.

Micro-DSC: The measurements were taken in TA multi-cell micro differential scanning calorimeter (micro-DSC). For this, $1.0 \mathrm{~mL}$ polymer solutions $(c=2.5-5.0 \mathrm{mg} / \mathrm{mL}$ in $1: 9 \mathrm{THF}$ : water) were prepared following the procedures discribed below and transferred to the designed DSC pan for the machine, with a sealed cap. For the reference pan, $1 \mathrm{~mL} \mathrm{1:9} \mathrm{THF:water} \mathrm{was} \mathrm{used.}$ The samples were initially cooled to $5{ }^{\circ} \mathrm{C}$ and then subjected to one heating / cooling cycle from 5 ${ }^{\circ} \mathrm{C}$ to $70{ }^{\circ} \mathrm{C}$ and back with a rate of $0.1{ }^{\circ} \mathrm{C} \mathrm{min}^{-1}$. The data presented, represents the first heating and second cooling runs.

Dynamic Light Scattering (DLS) measurements were performed using a Malvern $\mu V$ Zetasizer equipped with an $830 \mathrm{~nm}$ laser and a scattering angle of $90^{\circ}$ at a temperature of $25^{\circ} \mathrm{C}$. Samples were prepared at $1 \mathrm{mg} / \mathrm{mL}$ and held in Sarstedt UV-transparent disposable cuvettes with a path length of $10 \times 2 \mathrm{~mm}$. Measurements were analyzed using Zetasizer software provided by Malvern Instruments to derive the correlation functions and the distributions of the hydrodynamic diameter.

Multi-Angle Dynamic Light Scattering (DLS) and Static Light Scattering (SLS) measurements were performed on an ALV ALVCGS-3 Compact Goniometer equipped with ALV5000 digital correlator and a green laser operating at $\lambda=532 \mathrm{~nm}$. Scattering intensity was detected over the angular range of 30 to 150 degrees with steps of 10 degrees, with 3 runs of 30 seconds per angle. Samples were prepared at 2.0 or $0.25 \mathrm{mg} / \mathrm{mL}$, held in Wilmad-Labglass high throughput nuclear magnetic resonance (NMR) sample tubes with an outer diameter of $5 \mathrm{~mm}$, and measured at $25^{\circ} \mathrm{C}$. For DLS AfterALV software provided by Dullware Inc. was used to derive the hydrodynamic diameter. For SLS, scattering intensity was averaged for each angle over 3 runs of 30 seconds per angle.

Fluorescence spectroscopy was performed on a Safire II multi-mode plate reader using an excitation wavelength of $550 \mathrm{~nm}$ and an emission range of $595-675 \mathrm{~nm}$ in steps of $5 \mathrm{~nm}$. A black flat bottom 96-wells plate was used to prepare a dilution series of previously assembled samples. 
$100 \mu \mathrm{L}$ of each sample was placed in the first well, after which a dilution series was made with MilliQ. To all wells $2 \mu \mathrm{L}$ Nile Red from a $50 \mu \mathrm{M}$ stock solution in $\mathrm{MeOH}$ was added and the samples were equilibrated for one hour.

TIRF microscopy: Total internal reflection fluorescence (TIRF) spectroscopy was performed using previously assembled samples with a concentration of $0.5-1 \mathrm{mg} / \mathrm{mL} .40 \mu \mathrm{L}$ of previously assembled samples were mixed with $0.5 \mu \mathrm{L}$ of a $1 \mathrm{mM}$ Nile Red stock solution in $\mathrm{MeOH}$ to achieve $5 \mathrm{~mol} \%$ of Nile Red present in the sample. The samples were transferred onto a cover slip and measured immediately. TIRF images were acquired with a Nikon N-STORM system. Nile Red was excited using a $561 \mathrm{~nm}$ laser. Fluorescence was collected by means of a Nikon $\times 100,1.4 \mathrm{NA}$ oil immersion objective and passed through a quad-band pass dichroic filter ( 97335 Nikon). Images were recorded with an EMCCD camera (ixon3, Andor, pixel size $0.17 \mu \mathrm{m}$ ).

Liquid chromatography-mass spectrometry (LC-MS) was performed on a Thermo Scientific LCQ Fleet. Samples were analyzed using a Shimadzu SCL-10 AD VP series HPLC coupled to a diode array detector (Finnigan Surveyor PDA Plus detector, Thermo Scientific) and an Ion-Trap (LCQ Fleet, Thermo Scientific). Analyses were performed using a reversed phase HPLC column (C4 Jupiter, $150 \times 2 \mathrm{~mm}, 5 \mu \mathrm{m}$ ), using an injection volume of $1-4 \mu \mathrm{L}$, a flow rate of $0.20 \mathrm{~mL} / \mathrm{min}$ and typically a gradient (20\% to $90 \%$ in $10 \mathrm{~min}$, held at $90 \%$ for 1 more minute) of acetonitrile in water (both containing $0.1 \%$ formic acid) at $298 \mathrm{~K}$.

Sample preparation procedures: Stock solutions of all BCOs were prepared according to the following procedure: $\mathrm{BCOs}$ were first dried overnight over $\mathrm{P}_{2} \mathrm{O}_{5}$ and at $40^{\circ} \mathrm{C}$ under high vacuum. Afterwards, the BCOs were dissolved in dichloromethane and the dissolution process was followed overtime to ensure that no crystalline regions were present. The solutions were filtered with $0.2 \mu \mathrm{m}$ PTFE filter to remove dust or aggregates. Ddichloromethane was removed via slow evaporation and subsequent drying at $40^{\circ} \mathrm{C}$ under high vacuum after which the BCOs were dissolved in THF at $10 \mathrm{mg} / \mathrm{mL}$ to obtain final stock solutions of the compounds. The stock solutions were used to obtain samples of the BCOs in water at a concentration of $1 \mathrm{mg} / \mathrm{mL}$ containing $10 \%$ THF.

Samples of $\mathrm{LLA}_{16} \mathrm{EO}_{48}$ and $\mathrm{LLA}_{8} \mathrm{EO}_{17}$ were prepared via a quick solvent switch to ensure the formation of small micelles. For this, $540 \mu \mathrm{L}$ of MiliQ water was placed in a vial in which $60 \mu \mathrm{L}$ of stock solution is injected during vortexing (15 seconds).

Samples of LLA16 $\mathrm{EO}_{11}$ were prepared via a slow solvent switch to ensure the formation of vesicles. For this, $60 \mu \mathrm{L}$ of the solution was placed in a vial. $540 \mu \mathrm{L}$ MilliQ water was added slowly using a Nexus 3000 Syringe pump $(0.05 \mathrm{~mL} / \mathrm{min})$ after which the sample was stirred overnight at $250 \mathrm{rpm}$.

Samples of $\mathrm{LLA}_{16} \mathrm{EO}_{17}$ were prepared via a slow solvent switch to ensure the formation of cylinders. For this, $60 \mu \mathrm{L}$ of the solution was placed in a vial. $540 \mu \mathrm{L}$ MilliQ water was added slowly using a Nexus 3000 Syringe pump $(0.05 \mathrm{~mL} / \mathrm{min})$ after which the sample was stirred overnight at $250 \mathrm{rpm}$. 


\section{Synthetic procedures}

TBDMS-LLA8-COOH and TBDMS-LLA16-Bn were synthesized as previously published. ${ }^{1}$

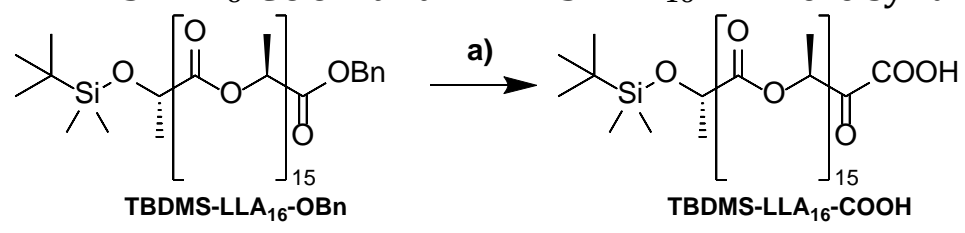
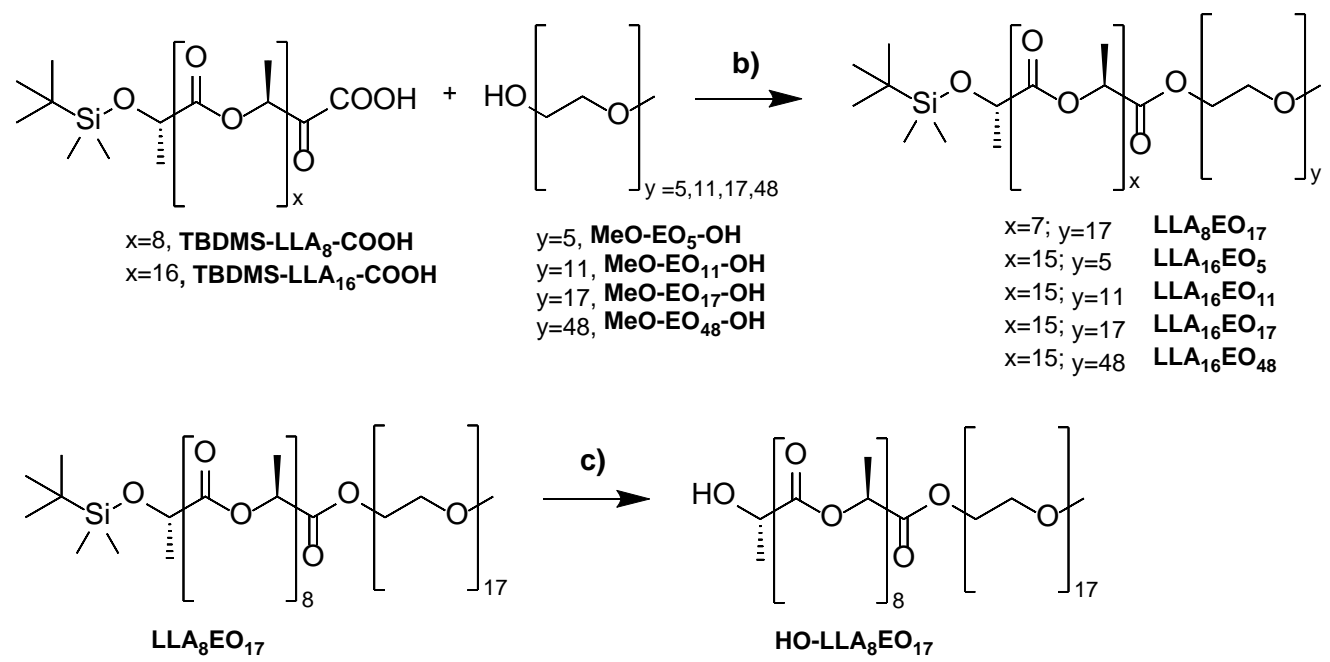

Scheme S1: Synthesis of the BCO library. a) H2, Pd/C, EtOAc, RT, 2 h, 98\%, b) EDC, DPTS, DCM, $0^{\circ} \mathrm{C}$-RTt, overnight, 39-66\%, c) $\mathrm{BF}_{3}$-etherate, $\mathrm{DCM}, 0^{\circ} \mathrm{C}-\mathrm{RT}$, overnight.

Synthesis of TBDMS-LLA16-COOH

TBDMS-LLA16-Bn (120 mg, $0.087 \mathrm{mmol})$ was dissolved in EtOAc $(1 \mathrm{~mL})$ and Pd/C was added (5 $\mathrm{mg}, 0.004 \mathrm{mmol}$ ). The reaction was stirred overnight until full conversion was reached. After filtering off the Pd/C and removal of the solvent, TBDMS-LLA16-COOH was obtained as a white solid (110 mg, $0.086 \mathrm{mmol}, 98 \%$ ).

${ }^{1} \mathrm{H}$ NMR (400 MHz, CDCl $): \delta=5.25-4.99\left(\mathrm{~m}, 15 \mathrm{H}, \mathrm{O}-\mathrm{CH}\left(\mathrm{CH}_{3}\right)-\right), 4.40$ (q, J = $6.7 \mathrm{~Hz}, 1 \mathrm{H}, \mathrm{HO}-$ $\left.\mathrm{CH}\left(\mathrm{CH}_{3}\right)-\mathrm{CO}\right), 1.64-1.49\left(\mathrm{~m}, 45 \mathrm{H}, \mathrm{O}-\mathrm{CH}\left(\mathrm{CH}_{3}\right)-\mathrm{CO}\right), 1.44\left(\mathrm{~d}, \mathrm{~J}=6.7 \mathrm{~Hz}, 3 \mathrm{H}, \mathrm{HO}-\mathrm{CH}\left(\mathrm{CH}_{3}\right)-\mathrm{CO}\right), 0.90$ $\left(\mathrm{s}, 9 \mathrm{H},\left(\mathrm{CH}_{3}\right)_{3}-\mathrm{C}-\mathrm{Si}\right), 0.09 \mathrm{ppm}\left(\mathrm{d}, \mathrm{J}=9.2 \mathrm{~Hz}, 6 \mathrm{H},\left(\mathrm{CH}_{3}\right)_{3}-\mathrm{C}-\mathrm{Si}-\left(\mathrm{CH}_{3}\right)_{2}-\right) .{ }^{13} \mathrm{C} \mathrm{NMR}\left(100 \mathrm{MHz} \mathrm{CDCl}_{3}\right) \delta=$ $169.60,68.51$ (d, J = 101.6 Hz), 25.70, 21.22, 18.28, 16.65, -5.10 (d, J = 39.4 Hz). HRMS (MALDIToF): $m / z$ calcd for C61H86033Si+Na+: $1307.44[\mathrm{M}+\mathrm{Na}+]$, found $1307.44 ; \mathrm{m} / z$ calcd for $\mathrm{C}_{61} \mathrm{H}_{86} \mathrm{O}_{33} \mathrm{Si}+\mathrm{K}^{+}: 1344.34[\mathrm{M}+\mathrm{K}]^{+}$, found 1344.34 .

Synthesis of $\mathrm{LLA}_{8} \mathrm{EO}_{17}$

TBDMS-LLA8-COOH (103 mg, $0.145 \mathrm{mmol})$ was dissolved in dry DCM $(0.57 \mathrm{~mL})$ in a roundbottom flask under an argon atmosphere. The solution was cooled to $0{ }^{\circ} \mathrm{C}$ in icewater and 4(dimethylamino)pyridinium 4-toluenesulfonate (DPTS, $8.11 \mathrm{mg}, 0.028 \mathrm{mmol}, 0.2 \mathrm{eq}$ ) and $\mathrm{N}$-(3dimethylaminopropyl)- $N$ '-ethylcarbodiimide hydrochloride (EDC $\cdot \mathrm{HCl}, 34.32 \mathrm{mg}, 66.2 \mathrm{mmol}, 1.3$ eq) were added. The mixture was stirred for $10 \mathrm{~min}$ at $0{ }^{\circ} \mathrm{C}$, followed by the addition of $\mathrm{MeO}$ $\mathrm{EO}_{17-} \mathrm{OH}(108 \mathrm{mg}, 0.179 \mathrm{mmol}, 1.009 \mathrm{eq}$ ). The resulting solution was stirred overnight at room temperature. The conversion was checked by TLC analysis (DCM/MeOH 93:7; CeMo stain). The reaction mixture was diluted with DCM $(5 \mathrm{~mL})$ and washed with brine $(5 \mathrm{~mL})$. The organic layer was dried with $\mathrm{MgSO}_{4}$ and concentrated in vacuo, giving the crude product $\mathrm{LLA}_{8} \mathrm{EO}_{17}$. Purification by automated column chromatography using DCM/MeOH (gradient 100/0 to 93/7) as eluent, gave the pure material $\mathrm{LLA}_{8} \mathrm{EO}_{17}(116.7 \mathrm{mg}, 58 \%)$. 
${ }^{1} \mathrm{H}$ NMR (399 MHz, CDCl 3$) \delta 5.15-4.99(\mathrm{~m}, 7 \mathrm{H}), 3.64-3.42(\mathrm{~m}, 64 \mathrm{H}), 3.30(\mathrm{~s}, 3 \mathrm{H}), 1.58-1.31(\mathrm{~m}$, $25 \mathrm{H}), 0.82(\mathrm{~s}, 9 \mathrm{H}), 0.06(\mathrm{~s}, 6 \mathrm{H})$. HRMS (MALDI-ToF): $\mathrm{m} / z$ calcd for $\mathrm{C}_{65} \mathrm{H}_{118} \mathrm{O}_{34} \mathrm{Si}+\mathrm{Na}^{+}: 1493.73$ $[\mathrm{M}+\mathrm{Na}]^{+}$, found 1493.71 .

Synthesis of LLA16EO5

TBDMS-LLA16-COOH (50 mg, $0.039 \mathrm{mmol}$ ) and DPTS (2.1 mg, $0.007 \mathrm{mmol})$ were dissolved in dry DCM $(0.5 \mathrm{ml})$. The mixture was cooled to $0^{\circ} \mathrm{C}$, and $\mathrm{EDC} \cdot \mathrm{HCl}(8.8 \mathrm{mg}, 0.046 \mathrm{mmol})$ was added. After 10 minutes $\mathrm{MeO}-\mathrm{EO}_{5}-\mathrm{OH}(8.9 \mathrm{mg}, 0.035 \mathrm{mmol}$ ) was added. The reaction was stirred overnight at room temperature when full conversion was reached $\left(\mathrm{R}_{\mathrm{f} \text {, product }}=0.42\right)$, giving crude $\mathrm{LLA}_{16} \mathrm{EO}_{5}$ (50 mg) as a colorless oil. The material was purified by automated column chromatography with $\mathrm{DCM} / \mathrm{MeOH}$ (gradient $100 / 0$ to $90 / 10$ over $10 \mathrm{CV}$, followed by $90 / 10$ to $80 / 20$ over $5 \mathrm{CV}$ ) as eluent, giving the pure material $\mathrm{LLA}_{16} \mathrm{EO}_{5}$ as a waxy solid (35.5 mg, 0.023 $\mathrm{mmol}, 66 \%)$.

${ }^{1} \mathrm{H}$ NMR (400 MHz, $\left.\mathrm{CDCl}_{3}\right) \delta=5.15\left(\mathrm{p}, \mathrm{J}=7.0 \mathrm{~Hz}, 15 \mathrm{H}, \mathrm{O}-\mathrm{CH}\left(\mathrm{CH}_{3}\right)-\right.$ ), 4.39 (q, J = 6.7 Hz, 1H, Si-O$\left.\mathrm{CH}\left(\mathrm{CH}_{3}\right)-\mathrm{CO}\right), 4.33-4.21\left(\mathrm{~m}, 2 \mathrm{H}, \mathrm{CO}-\mathrm{O}-\mathrm{CH}_{2}-\mathrm{CH}_{2}-\right), 3.72-3.57\left(\mathrm{~m}, 16 \mathrm{H}, \mathrm{CH}_{3}-\left(\mathrm{O}-\left(\mathrm{CH}_{2}\right)_{2}\right) 4\right), 3.54$ (dd, J = 5.9, $\left.\left.3.5 \mathrm{~Hz}, 2 \mathrm{H}, \mathrm{CO}-\mathrm{O}-\mathrm{CH}_{2}-\mathrm{CH}_{2}-\right), 3.37\left(\mathrm{~s}, 3 \mathrm{H}, \mathrm{CH}_{3}-\mathrm{O}-\mathrm{CH}_{2}\right)_{2}-\mathrm{O}\right), 1.64-1.48(\mathrm{~m}, 45 \mathrm{H}, 0-$ $\left.\mathrm{CH}\left(\mathrm{CH}_{3}\right)-\mathrm{CO}\right), 1.44$ (d, J = $\left.6.8 \mathrm{~Hz}, 3 \mathrm{H}, \mathrm{Si}-\mathrm{O}-\mathrm{CH}\left(\mathrm{CH}_{3}\right)-\mathrm{CO}\right), 0.89$ (s, 9H, (CH3) $\left.3-\mathrm{C}-\mathrm{Si}\right), 0.09$ (d, J = 9.3 $\left.\mathrm{Hz}, 6 \mathrm{H},\left(\mathrm{CH}_{3}\right)_{3}-\mathrm{C}-\mathrm{Si}-\left(\mathrm{CH}_{3}\right)_{2}-\right) .{ }^{13} \mathrm{C} \mathrm{NMR}\left(100 \mathrm{MHz}, \mathrm{CDCl}_{3}\right) \delta=173.52,170.83-166.91(\mathrm{~m}), 71.93$, $71.09-70.21(\mathrm{~m}), 69.39-68.34(\mathrm{~m}), 67.99,64.45,59.03,25.69,21.22,18.28,17.49-15.22(\mathrm{~m})$, $5.10 \mathrm{ppm}(\mathrm{d}, \mathrm{J}=39.3 \mathrm{~Hz})$; HRMS (MALDI-ToF): $\mathrm{m} / \mathrm{z}$ calcd for C65H102038Si+Na ${ }^{+} 1542.58$ $[\mathrm{M}+\mathrm{Na}]^{+}$, found 1541.58; $\mathrm{m} / z$ calcd for $\mathrm{C}_{65} \mathrm{H}_{102} \mathrm{O}_{38} \mathrm{Si}+\mathrm{K}^{+}: 1557.58[\mathrm{M}+\mathrm{K}]^{+}$, found 1557.55; 24

Synthesis of $L L A_{16} E O_{11}$

TBDMS-LLA16-COOH (50 mg, $0.039 \mathrm{mmol})$ and DPTS (2.1 $\mathrm{mg}, 0.007 \mathrm{mmol}$ ) were dissolved in dry DCM $(0.5 \mathrm{ml})$. The mixture was cooled to $0^{\circ} \mathrm{C}$, and $\mathrm{EDC} \cdot \mathrm{HCl}(8.8 \mathrm{mg}, 0.046 \mathrm{mmol})$ was added. After 10 minutes $\mathrm{MeO}-\mathrm{EO}_{11}-\mathrm{OH}(18.3 \mathrm{mg}, 0.035 \mathrm{mmol})$ was added. The reaction was stirred overnight at room temperature when full conversion was reached $\left(\mathrm{R}_{\mathrm{f}}\right.$, product $\left.=0.25\right)$, giving crude LLA $_{16} \mathrm{EO}_{11}(50 \mathrm{mg}$ ) as a colorless oil. The material was purified by automated column chromatography with $\mathrm{DCM} / \mathrm{MeOH}$ (gradient $100 / 0$ to $90 / 10$ over $10 \mathrm{CV}$, followed by $90 / 10$ to $80 / 20$ over $5 \mathrm{CV}$ ) as eluent, giving the pure material $\mathrm{LLA}_{16} \mathrm{EO}_{11}$ as a colorless oil (31.9 mg, 0.018 $\mathrm{mmol}, 51 \%)$.

${ }^{1} \mathrm{H}$ NMR (400 MHz, CDCl 3$) \delta=5.13\left(\mathrm{q}, \mathrm{J}=7.0 \mathrm{~Hz}, 15 \mathrm{H}, \mathrm{O}-\mathrm{CH}\left(\mathrm{CH}_{3}\right)-\right.$ ), $4.36(\mathrm{q}, \mathrm{J}=6.8 \mathrm{~Hz}, 1 \mathrm{H}, \mathrm{Si}-\mathrm{O}-$ $\left.\mathrm{CH}\left(\mathrm{CH}_{3}\right)-\mathrm{CO}\right), 4.32-4.17\left(\mathrm{~m}, 2 \mathrm{H}, \mathrm{CO}-\mathrm{O}-\mathrm{CH}_{2}-\mathrm{CH}_{2}-\right), 3.72-3.54\left(\mathrm{~m}, 40 \mathrm{H}, \mathrm{CH}_{3}-\left(\mathrm{O}-\left(\mathrm{CH}_{2}\right) 2\right) 10\right), 3.54-$ 3.48 (m, 2H, CO-O-CH2-CH2-), 3.34 (s, 3H, CH3-O-CH2)2-O), $1.63-1.44\left(\mathrm{~m}, 45 \mathrm{H}, 0-\mathrm{CH}_{2}\left(\mathrm{CH}_{3}\right)-\mathrm{CO}\right)$, $1.41\left(\mathrm{~d}, \mathrm{~J}=6.8 \mathrm{~Hz}, 3 \mathrm{H}, \mathrm{Si}-\mathrm{O}-\mathrm{CH}\left(\mathrm{CH}_{3}\right)-\mathrm{CO}\right), 0.86\left(\mathrm{~s}, 9 \mathrm{H},\left(\mathrm{CH}_{3}\right)_{3}-\mathrm{C}-\mathrm{Si}\right), 0.09$ (d, J = 9.3 Hz, 6H, $\left(\mathrm{CH}_{3}\right)_{3}-$ $\left.\mathrm{C}-\mathrm{Si}-\left(\mathrm{CH}_{3}\right)_{2}-\right) .{ }^{13} \mathrm{C}$ NMR $\left(100 \mathrm{MHz} \mathrm{CDCl}_{3}\right) \delta=173.51,171.48-167.04(\mathrm{~m}), 71.92,70.56,69.42-$ $68.44(\mathrm{~m}), 67.98,64.43,59.01,25.69,21.21,18.26,16.86-15.74(\mathrm{~m}),-5.11 \mathrm{ppm}(\mathrm{d}, \mathrm{J}=39.3 \mathrm{~Hz})$; HRMS (MALDI-ToF): $m / z$ calcd for C77H126044Si+Na+: $1806.9[\mathrm{M}+\mathrm{Na}]^{+}$, found 1805.73; $\mathrm{m} / \mathrm{z}$ calcd for $\mathrm{C}_{77} \mathrm{H}_{126} \mathrm{O}_{44} \mathrm{Si}+\mathrm{K}^{+}: 1822.9[\mathrm{M}+\mathrm{K}]^{+}$, found 1821.7 .

Synthesis of $L L A_{16} E O_{17}$

TBDMS-LLA16-COOH (50 mg, $0.039 \mathrm{mmol}$ ) and DPTS (2.1 $\mathrm{mg}, 0.007 \mathrm{mmol})$ were dissolved in dry DCM $(0.5 \mathrm{~mL})$. The mixture was cooled to $0^{\circ} \mathrm{C}$, and $\mathrm{EDC} \cdot \mathrm{HCl}(8.8 \mathrm{mg}, 0.046 \mathrm{mmol})$ was added. After 10 minutes $\mathrm{MeO}-\mathrm{EO}_{17}-\mathrm{OH}(27 \mathrm{mg}, 0.035 \mathrm{mmol})$ was added. The reaction was stirred overnight at room temperature when full conversion was reached $\left(\mathrm{R}_{\mathrm{f}}\right.$, product $\left.=0.18\right)$, giving crude $\mathrm{LLA}_{16} \mathrm{EO}_{17}$ (72 mg) as a colorless oil. The material was purified by automated column chromatography with $\mathrm{DCM} / \mathrm{MeOH}$ (gradient $100 / 0$ to $90 / 10$ over $10 \mathrm{CV}$, followed by $90 / 10$ to 
80/20 over $5 \mathrm{CV}$ ) as eluent, giving the pure material LLA16EO17 as an oil (31 $\mathrm{mg}, 0.015 \mathrm{mmol}$, $39 \%)$.

${ }^{1} \mathrm{H}$ NMR (400 MHz, $\left.\mathrm{CDCl}_{3}\right) \delta=5.14\left(\mathrm{p}, \mathrm{J}=7.0 \mathrm{~Hz}, 15 \mathrm{H}, \mathrm{O}-\mathrm{CH}\left(\mathrm{CH}_{3}\right)-\right.$ ), $4.38(\mathrm{q}, \mathrm{J}=6.8 \mathrm{~Hz}, 1 \mathrm{H}, \mathrm{Si}-\mathrm{O}-$ $\left.\mathrm{CH}\left(\mathrm{CH}_{3}\right)-\mathrm{CO}\right), 4.33-4.19\left(\mathrm{~m}, 2 \mathrm{H}, \mathrm{CO}-\mathrm{O}-\mathrm{CH}_{2}-\mathrm{CH}_{2}-\right), 3.71-3.56\left(\mathrm{~m}, 64 \mathrm{H}, \mathrm{CH}_{3}-\left(\mathrm{O}-\left(\mathrm{CH}_{2}\right)_{2}\right)_{16}\right), 3.56-$ 3.46 (m, 2H, CO-O-CH2- $\mathrm{CH}_{2}-$ ), 3.37 (s, 3H, CH3-O-CH2) $\left.2-\mathrm{O}\right), 1.66-1.46\left(\mathrm{~m}, 45 \mathrm{H}, \mathrm{O}-\mathrm{CH}\left(\mathrm{CH}_{3}\right)-\mathrm{CO}\right)$, $1.43\left(\mathrm{~d}, \mathrm{~J}=6.8 \mathrm{~Hz}, 3 \mathrm{H}, \mathrm{Si}-\mathrm{O}-\mathrm{CH}\left(\mathrm{CH}_{3}\right)-\mathrm{CO}\right), 0.89$ (s, 9H, (CH3) $\left.3-\mathrm{C}-\mathrm{Si}\right), 0.09$ (d, J = $9.3 \mathrm{~Hz}, 6 \mathrm{H},\left(\mathrm{CH}_{3}\right)_{3}-$ $\left.\mathrm{C}-\mathrm{Si}-\left(\mathrm{CH}_{3}\right)_{2}-\right) .{ }^{13} \mathrm{C} \mathrm{NMR}\left(100 \mathrm{MHz}, \mathrm{CDCl}_{3}\right) \delta=173.52,172.08-167.56(\mathrm{~m}), 71.94,70.58,69.38-$ 68.32 (m), 67.99, 64.44, 59.04, 25.69, 21.22, 18.28, 17.39 - $15.73(\mathrm{~m}),-5.10 \mathrm{ppm}(\mathrm{d}, \mathrm{J}=39.3 \mathrm{~Hz})$; HRMS (MALDI-ToF $m / z$ calcd for $\mathrm{C}_{89} \mathrm{H}_{150} \mathrm{O}_{50} \mathrm{Si}+\mathrm{Na}^{+}: 2069.9[\mathrm{M}+\mathrm{K}]^{+}$, found 2069.84 .

Synthesis of $L L A_{16} E_{48}$

TBDMS-LLA16-COOH (36 mg, $0.028 \mathrm{mmol}$ ) and DPTS (1.4 mg, $0.005 \mathrm{mmol}$ ) were dissolved in dry DCM $(0.5 \mathrm{ml})$. The mixture was cooled to $0^{\circ} \mathrm{C}$, and EDC $\cdot \mathrm{HCl}(6 \mathrm{mg}, 0.032 \mathrm{mmol})$ was added. After 10 minutes HO-EG48-OMe (50 mg, $0.023 \mathrm{mmol}$ ) was added. The reaction was stirred overnight at room temperature when full conversion was reached $\left(\mathrm{R}_{\mathrm{f} \text {, product }}=0.5\right)$, giving crude $\mathrm{LLA}_{16} \mathrm{EO}_{48}(80$ $\mathrm{mg}$ ) as a colorless oil. The material was purified by automated column chromatography with $\mathrm{DCM} / \mathrm{MeOH}$ (gradient $100 / 0$ to $90 / 10$ over $20 \mathrm{CV}$ ) as eluent, giving the pure material $\mathrm{LLA}_{16} \mathrm{EO}_{48}$ as a white solid (49 $\mathrm{mg}, 0.014 \mathrm{mmol}, 61 \%$ ).

${ }^{1} \mathrm{H}$ NMR (400 MHz, CDCl 3 ) $\delta 5.16(\mathrm{~m}, 15 \mathrm{H}, \mathrm{CH}-\mathrm{LA}), 4.40$ (q, $J=6.8 \mathrm{~Hz}, 1 \mathrm{H}, \mathrm{CH}-\mathrm{LA}$ next to TBDMS), 4.28 (dt, $J=13.3,4.8 \mathrm{~Hz}, 2 \mathrm{H}, \mathrm{CH}_{2}$-PEG next to LA), 3.64 (s, 198H, CH2-PEG), 3.38 (s, 3H, CH3-PEG), $1.66-1.47$ (m, 47H, CH3-LA), 1.45 (d, J = 6.8 Hz, 3H, CH3-LA), 0.90 (s, 9H, CH3 TBDMS), 0.10 (d, $J=$ $9.4 \mathrm{~Hz}, 6 \mathrm{H}, \mathrm{CH} 2$ TBDMS). HRMS (MALDI-ToF): $m / z$ calcd for $\mathrm{C}_{151} \mathrm{H}_{274} \mathrm{O}_{81} \mathrm{Si}+\mathrm{Na}^{+}: 3436.86[\mathrm{M}+\mathrm{Na}]^{+}$, found 3436.71 .

Synthesis of $\mathrm{HO}-\mathrm{LLA}_{8} \mathrm{EO}_{17}$

$\mathrm{LLA}_{8} \mathrm{EO}_{17}(14.6 \mathrm{mg}, 0.0099 \mathrm{mmol})$ was dissolved in dry DCM (5 mL) in a round-bottom flask under an argon atmosphere. The solution was cooled down to $0{ }^{\circ} \mathrm{C}$ in icewater. Next, $\mathrm{BF}_{3}$-etherate (4.224 mg $0.02976 \mathrm{mmol}, 3 \mathrm{eq}$ ) was added slowly and the mixture was allowed to warm to room

temperature. Stirring was continued overnight at room temperature. Full conversion of the starting material was confirmed by TLC analysis (DCM/MeOH 93:7; CeMo stain). Subsequently, the solution was poured into a mixture of sat. $\mathrm{NaHCO}_{3}(4 \mathrm{~mL})$ and brine $(4 \mathrm{~mL})$. The organic layer was washed again with brine $(1 \mathrm{~mL})$ and dried with $\mathrm{MgSO}_{4}$. The solvent was removed in vacuo, giving product $\mathrm{HO}-\mathrm{LLA}_{8} \mathrm{EO}_{17}(10.3 \mathrm{mg}, 77 \%)$. The product is not $100 \%$ pure. It contains traces of starting material $\mathrm{LLA}_{8} \mathrm{EO}_{17}$.

${ }^{1} \mathrm{H}$ NMR (399 MHz, CDCl 3 ) $\delta 5.27-5.10(\mathrm{~m}, 7 \mathrm{H}), 4.41-4.18(\mathrm{~m}, 3 \mathrm{H}), 3.65(\mathrm{~d}, J=2.1 \mathrm{~Hz}, 61 \mathrm{H}), 3.59$ - $3.51(\mathrm{~m}, 2 \mathrm{H}), 3.38(\mathrm{~s}, 3 \mathrm{H}), 1.64$ - $1.46(\mathrm{~m}, 25 \mathrm{H})$. HRMS (MALDI-TOF): $\mathrm{m} / z$ calcd for C59H104034Si+Na ${ }^{+}: 1380.45[\mathrm{M}+\mathrm{Na}]^{+}$, found 1379.75. 


\section{Supporting Characterization Figures}

Figure S1a: MALDI-ToF-MS of LLA8EO 17 :

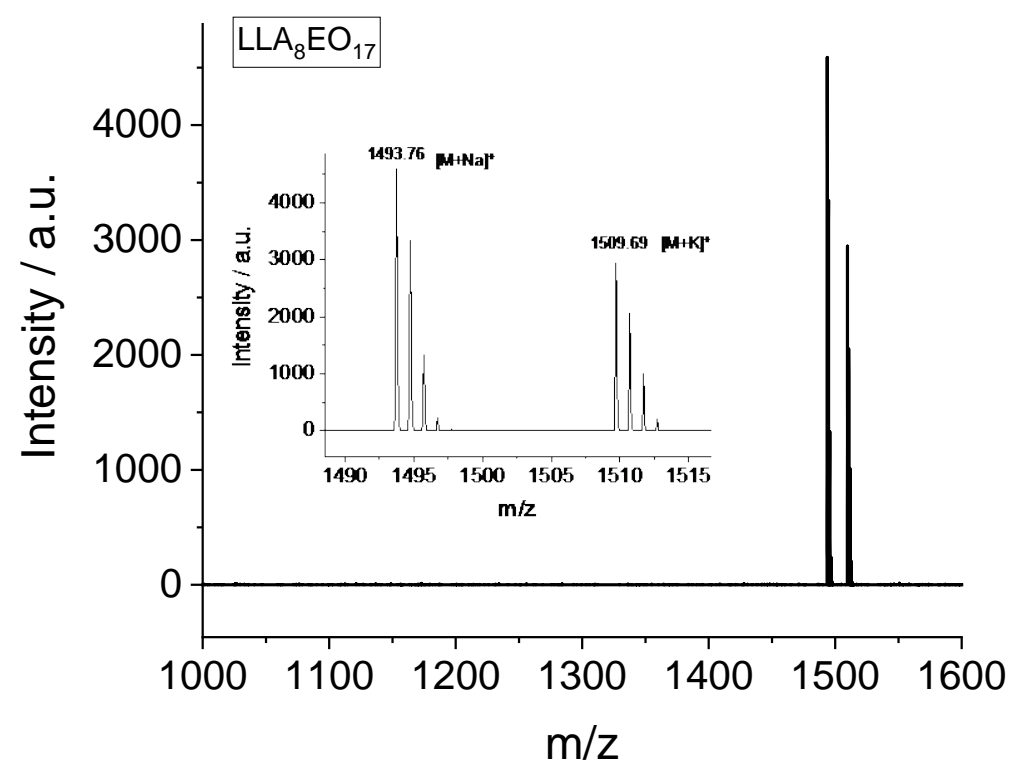

Figure S1b: ${ }^{1} \mathrm{H}-\mathrm{NMR}$ of $\mathbf{L L A}_{8} \mathbf{E O}_{17}$ :

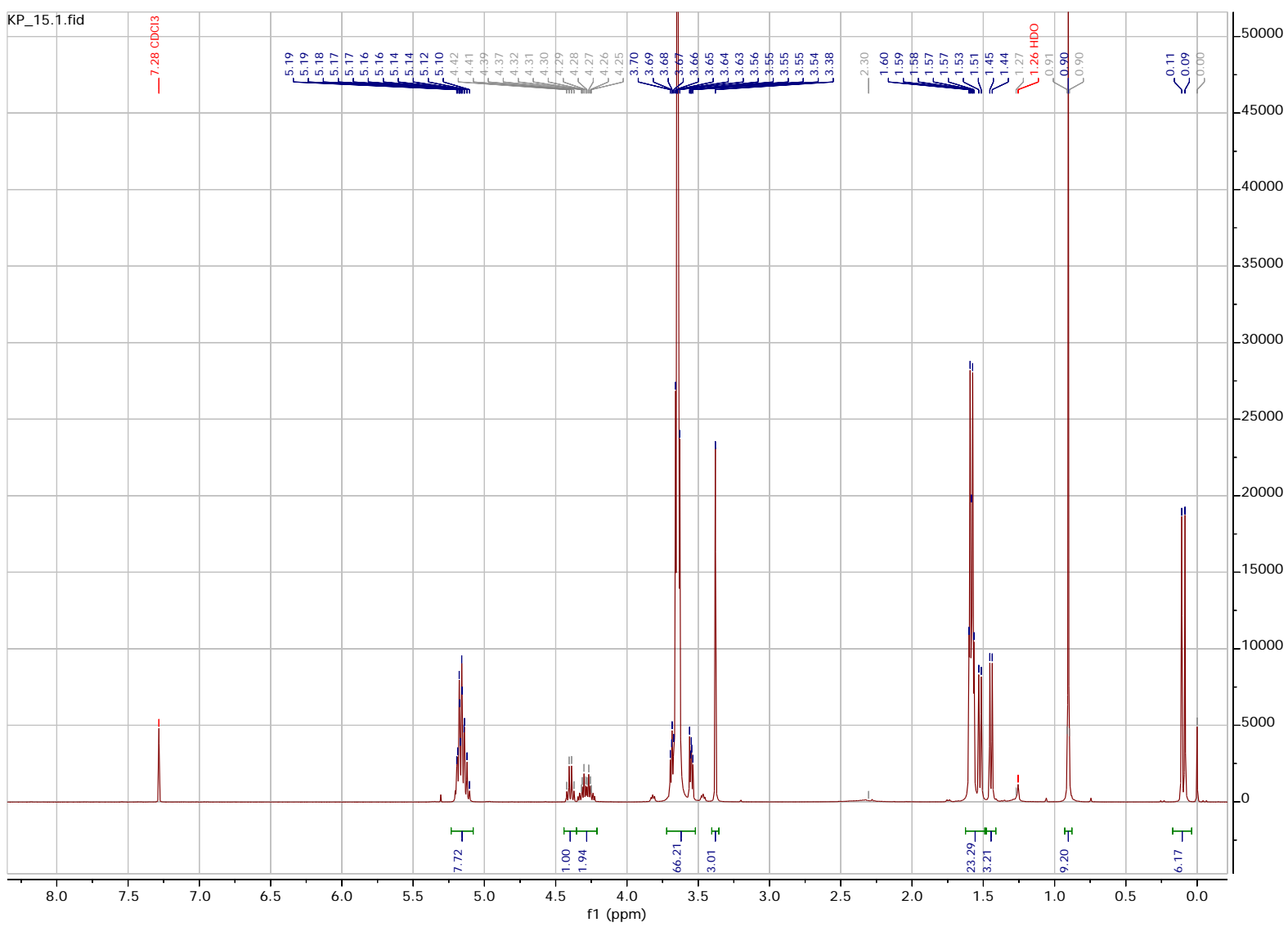


Figure S1c: gCOSY of LLA8EO 17 :

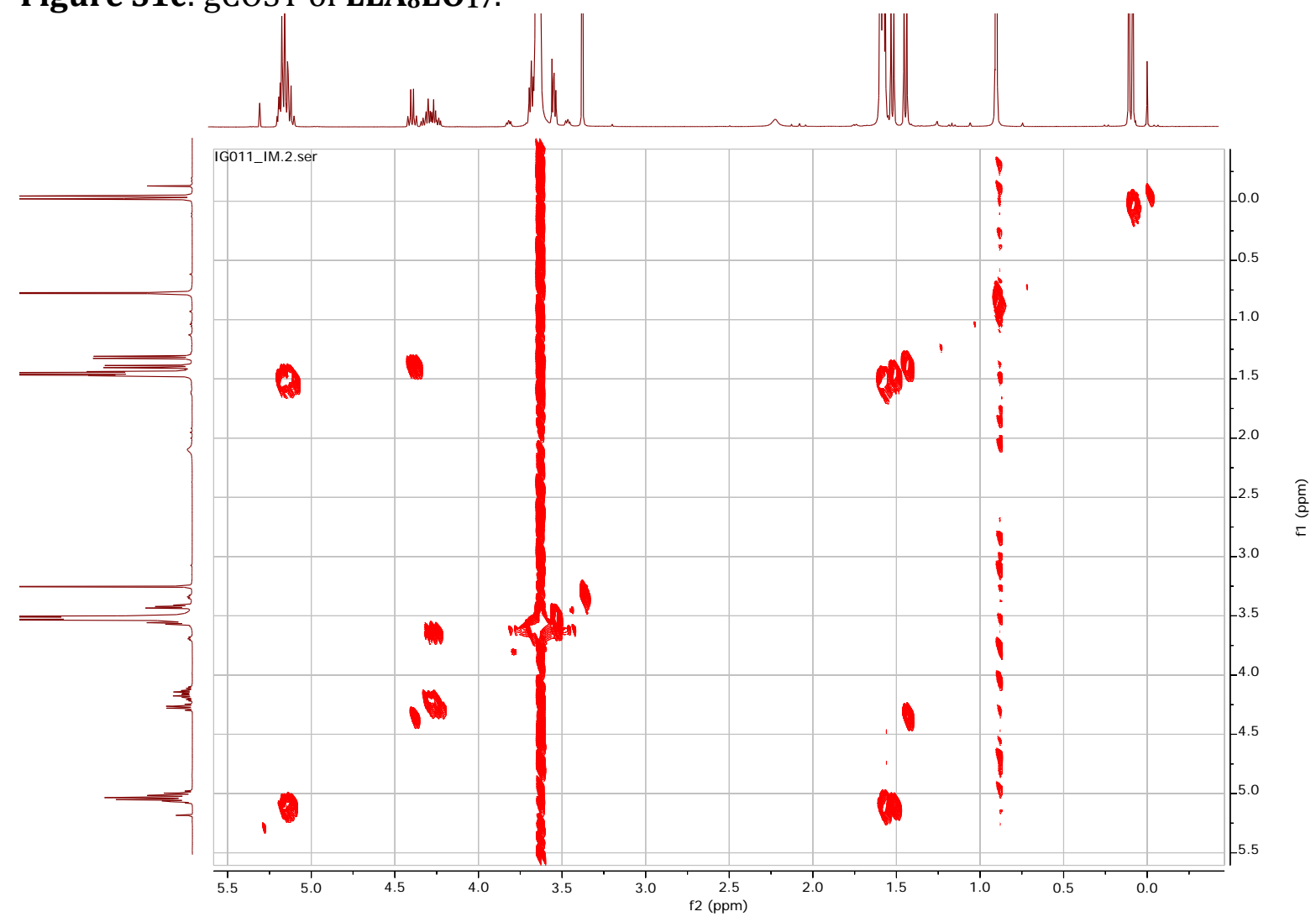

S1d: ${ }^{13}$ C NMR of LLA8EO17:

Figure

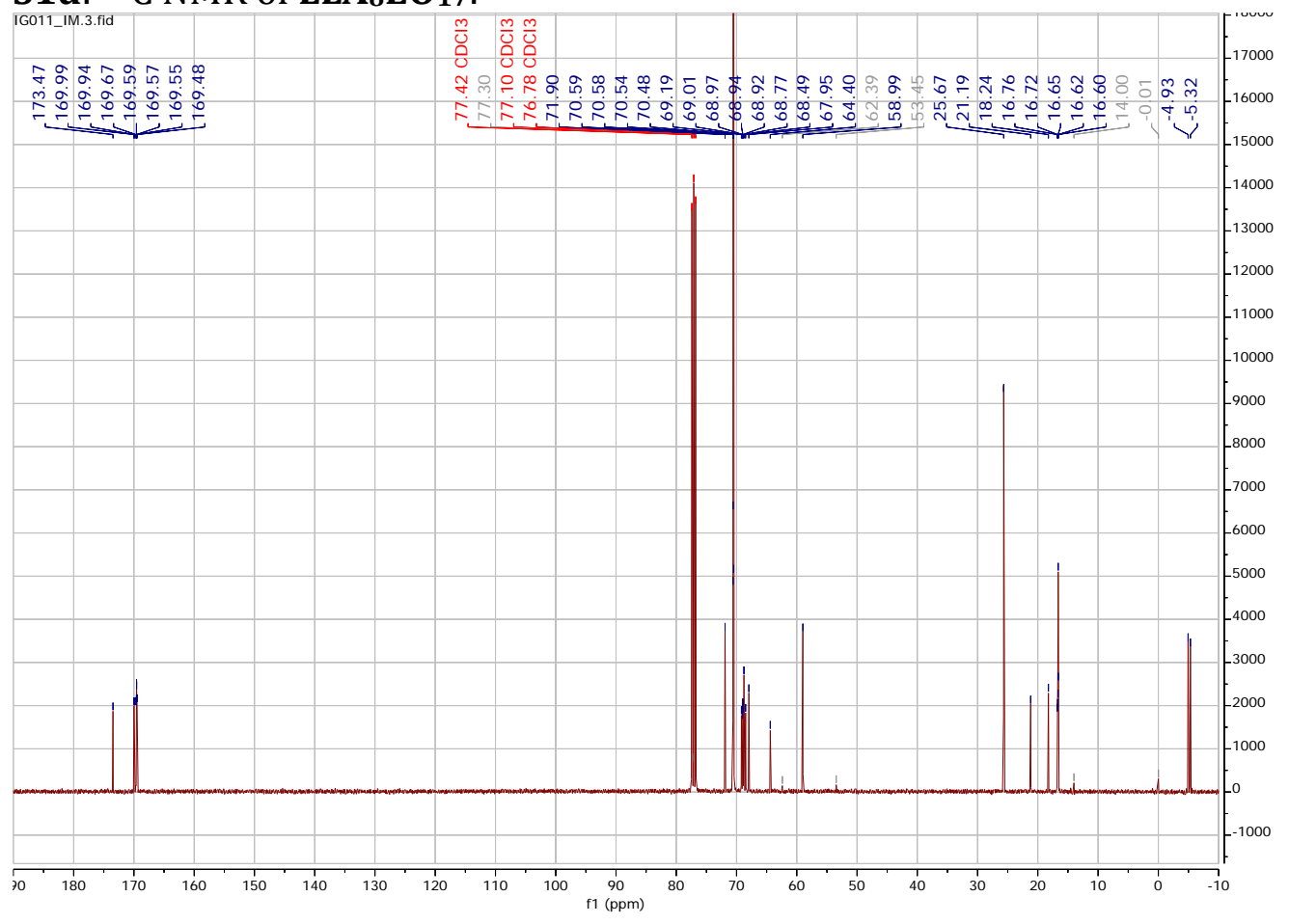


Figure S2a: MALDI-ToF of LLA16E05:

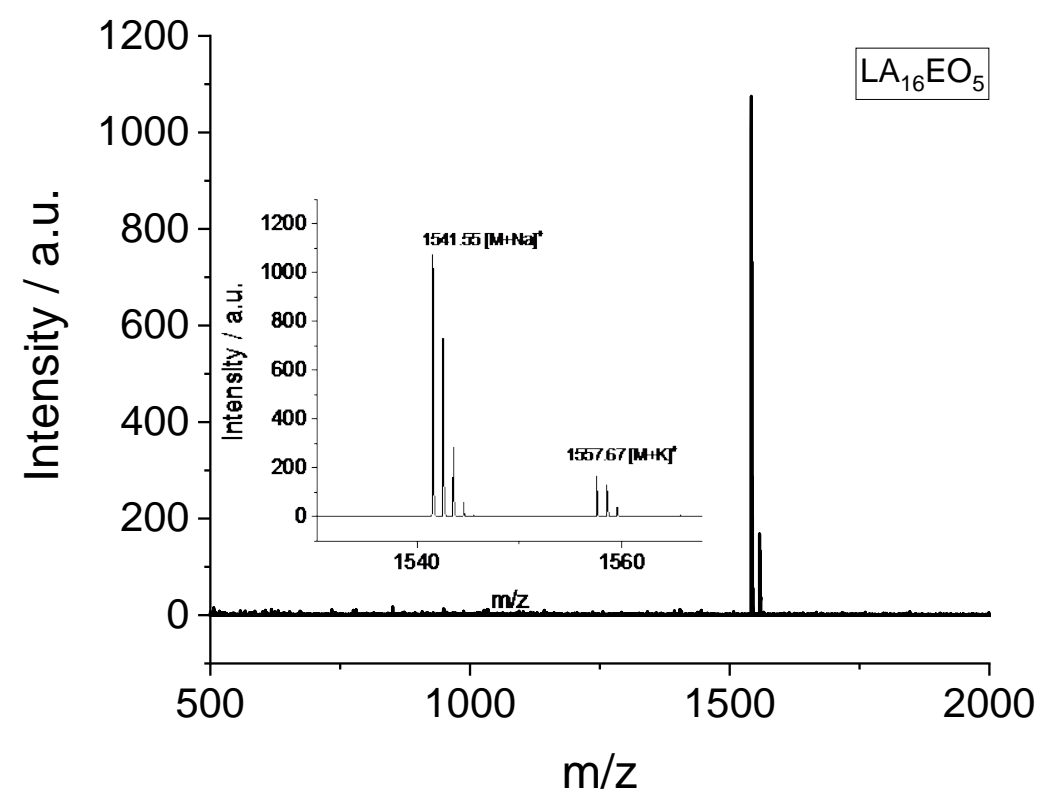

Figure S2b: ${ }^{1} \mathrm{H}$ NMR of LLA16EO5:

MA_16013_LA16EG5.1.fid

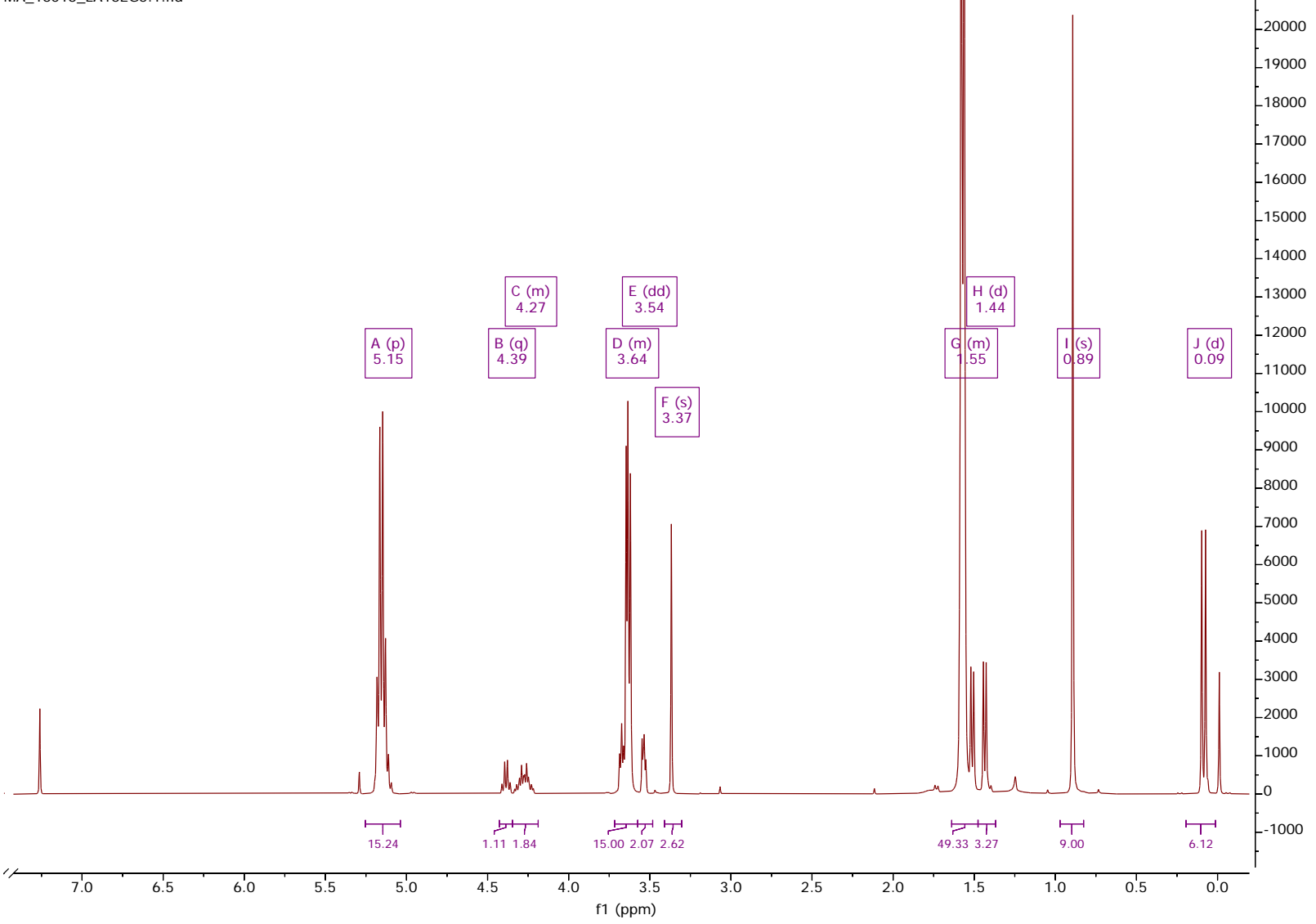


Figure S2c: ${ }^{13} \mathrm{C}$ NMR of LLA16EO5:

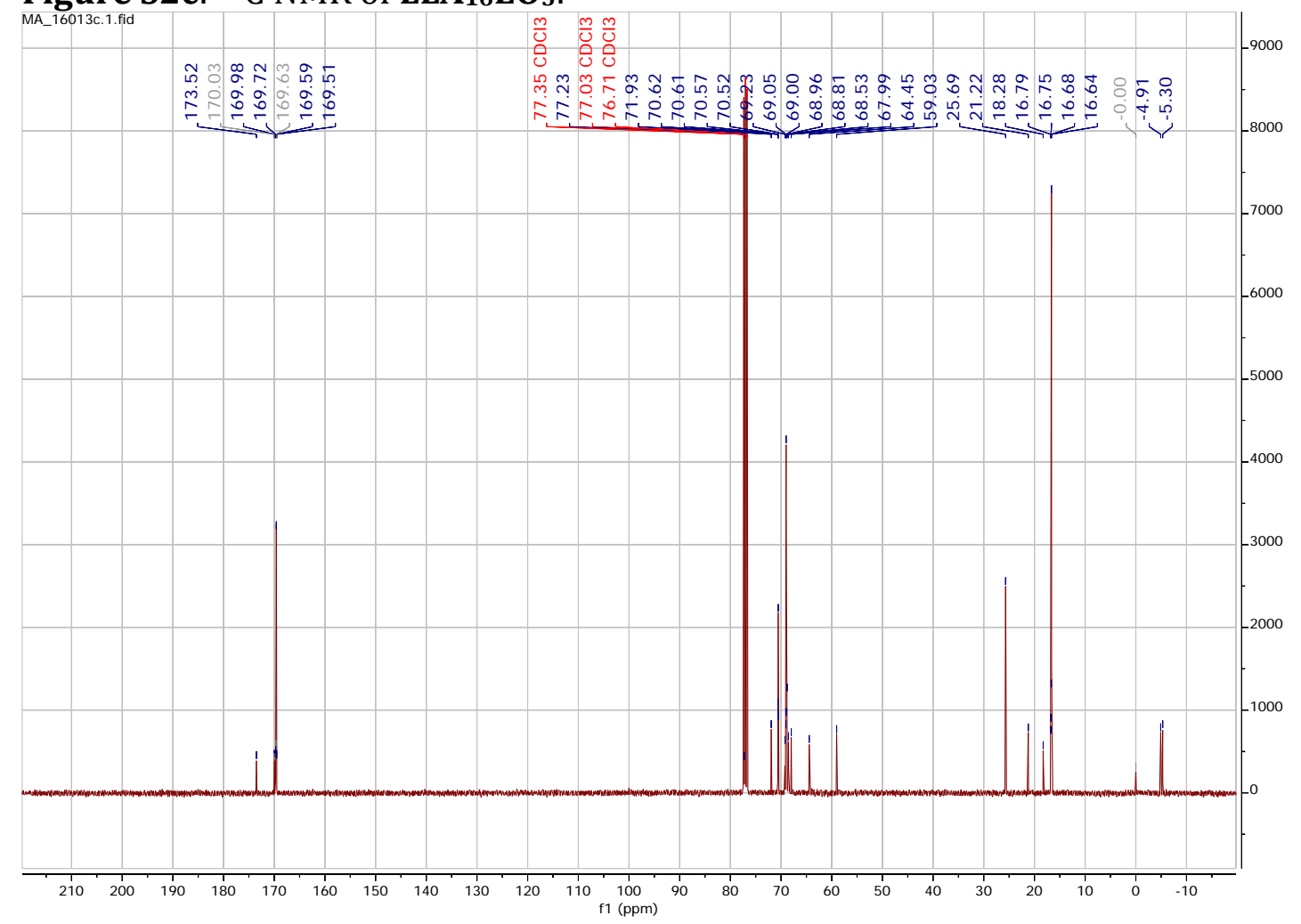

Figure S2d: HSQC NMR of LLA16E5:

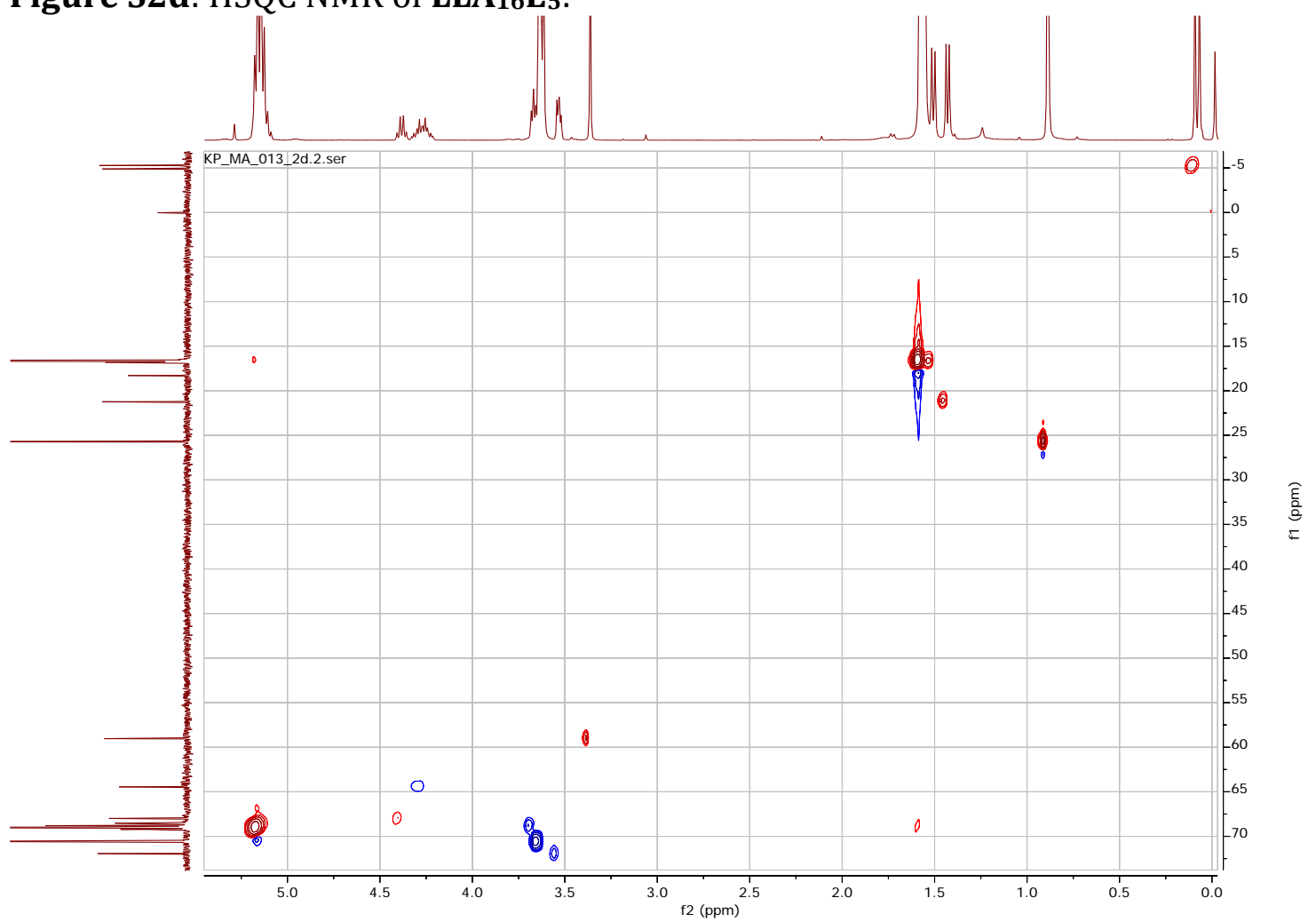


Figure S3a: MALDI-ToF of LLA16E011:

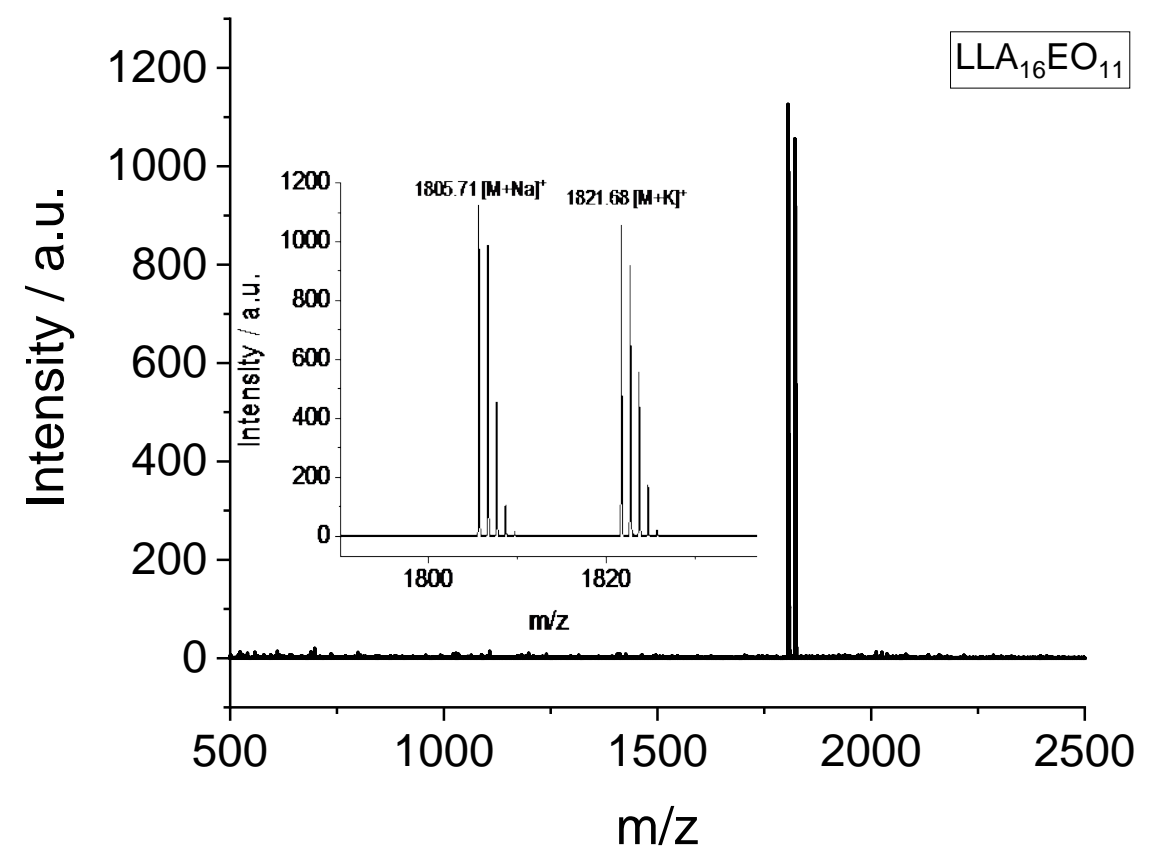

Figure S3b: ${ }^{1} \mathrm{H}$ NMR of LLA16EO ${ }_{11}$ :

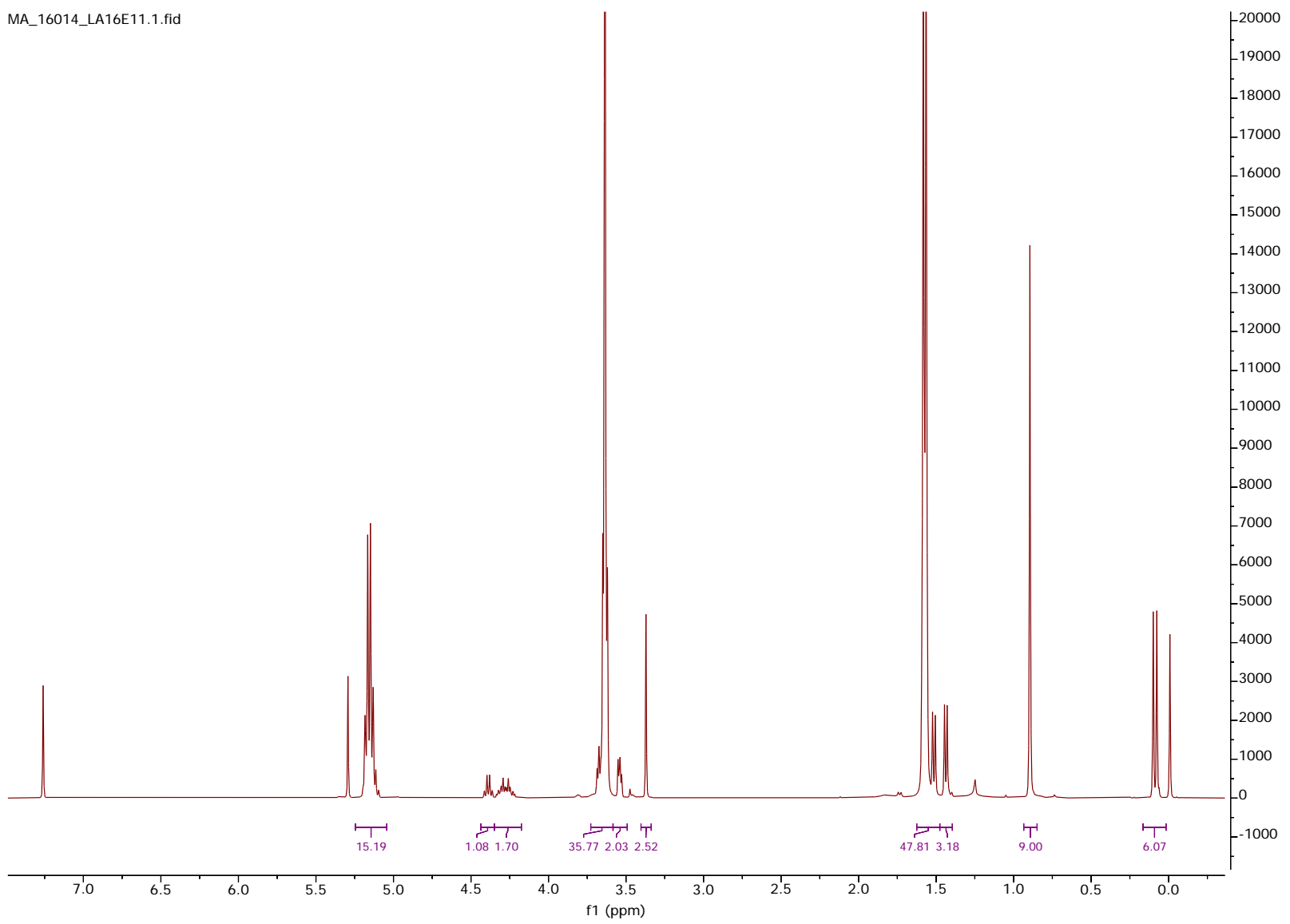


Figure S3c: ${ }^{13} \mathrm{C}$ NMR of LLA16EO11:

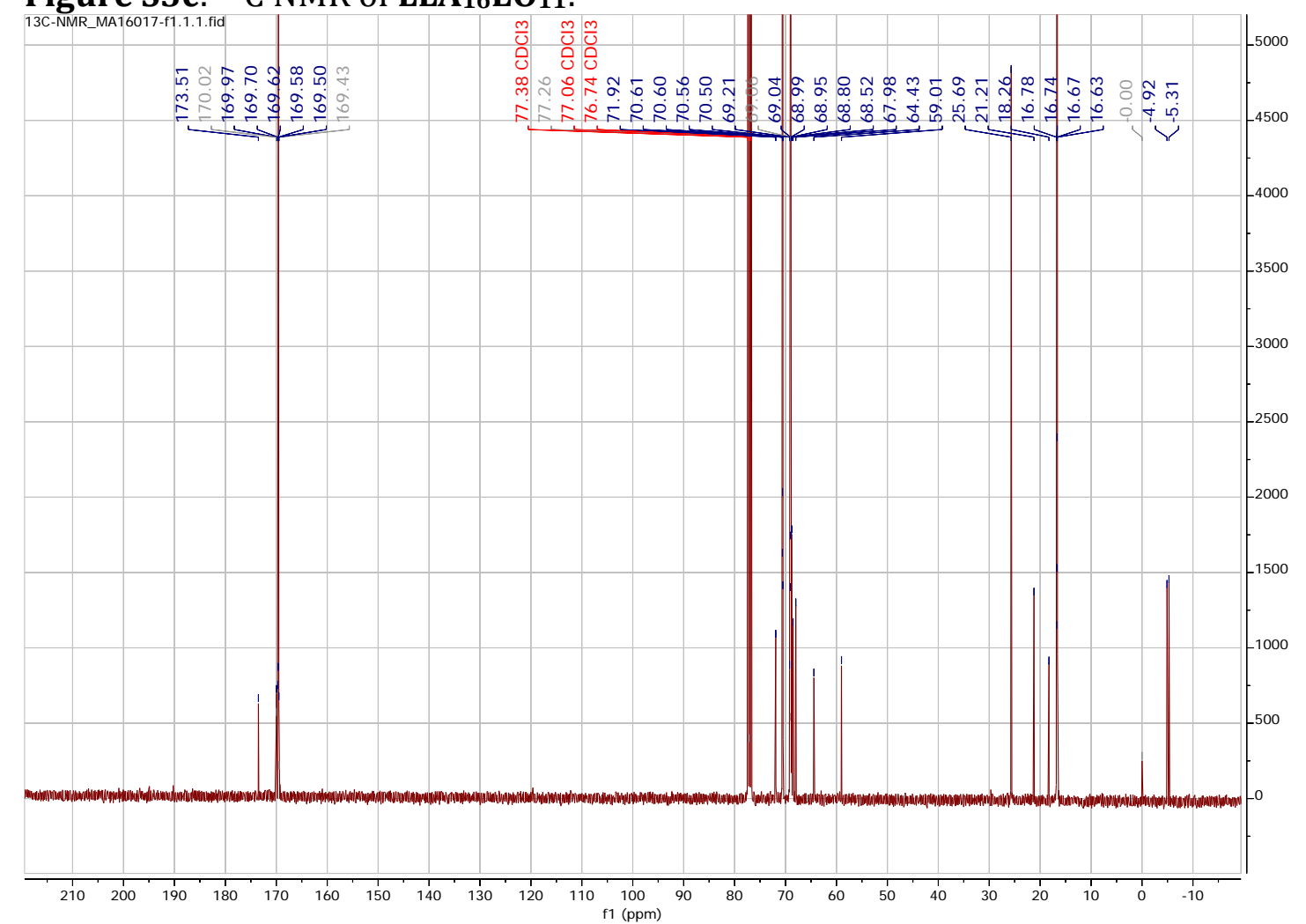

Figure S3d: HSQC NMR of LLA16EO11:

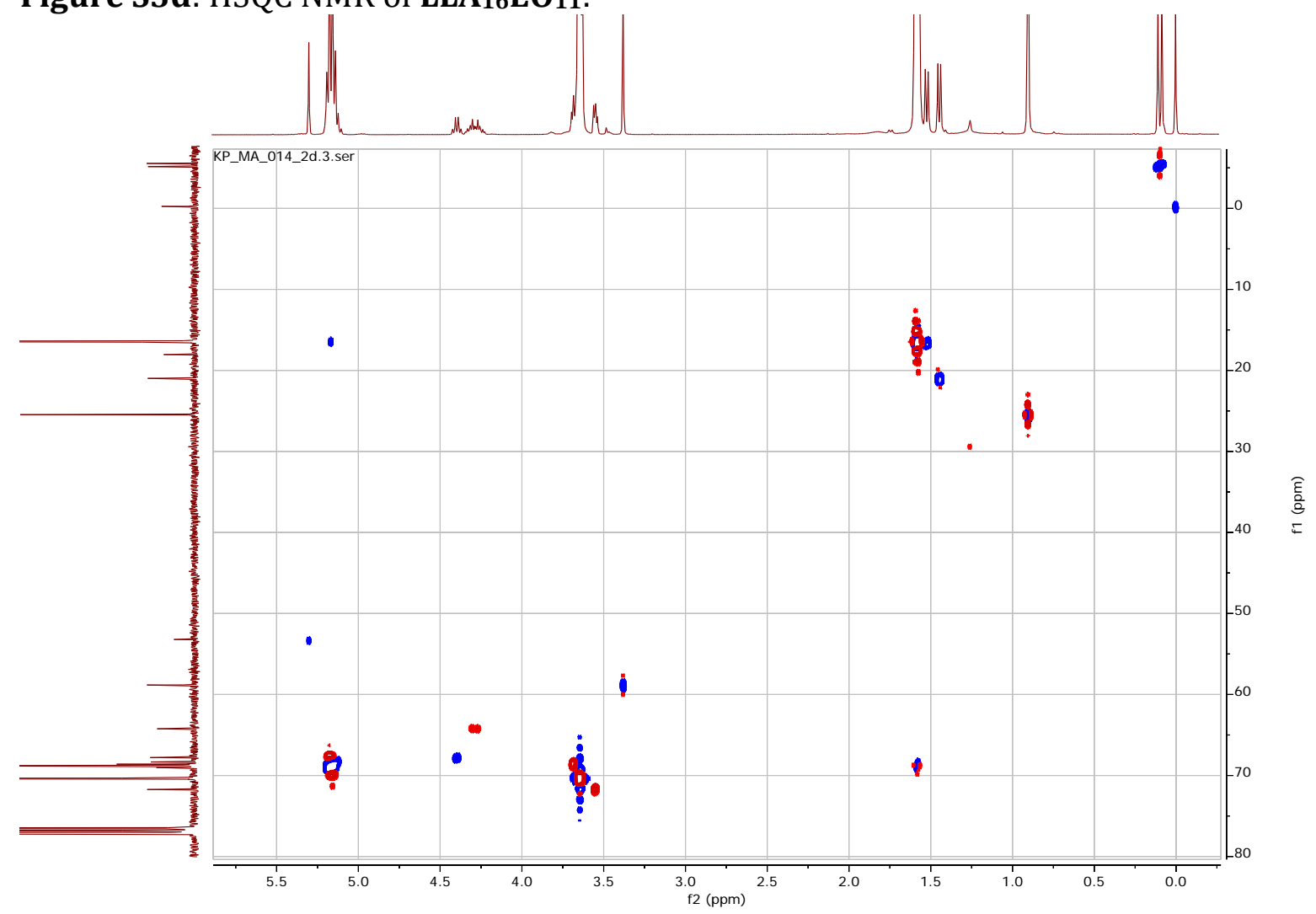


Figure S3e: gCOSY NMR of LLA16EO11:

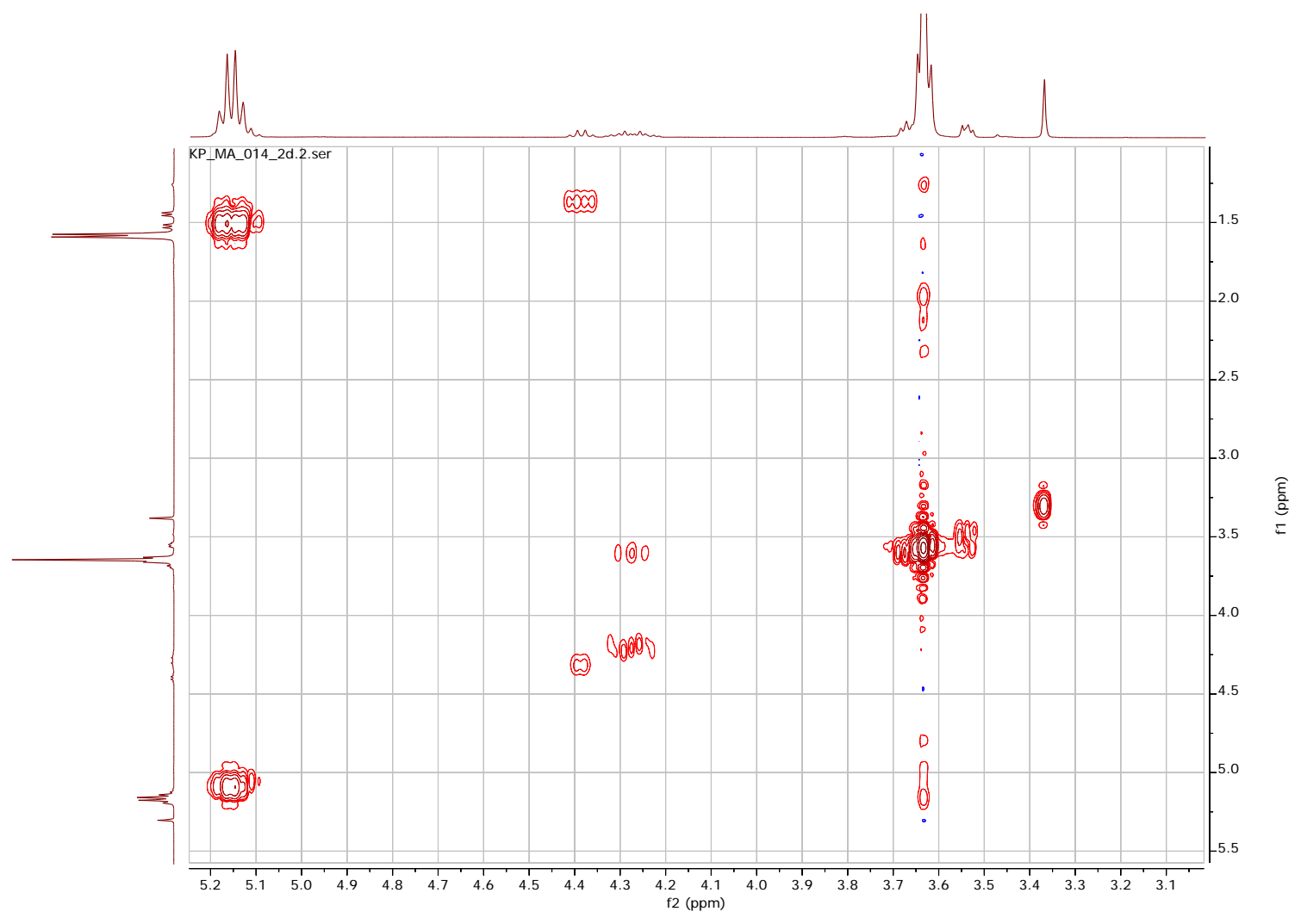

S14 
Figure S4a: MALDI-ToF of LLA16E017:

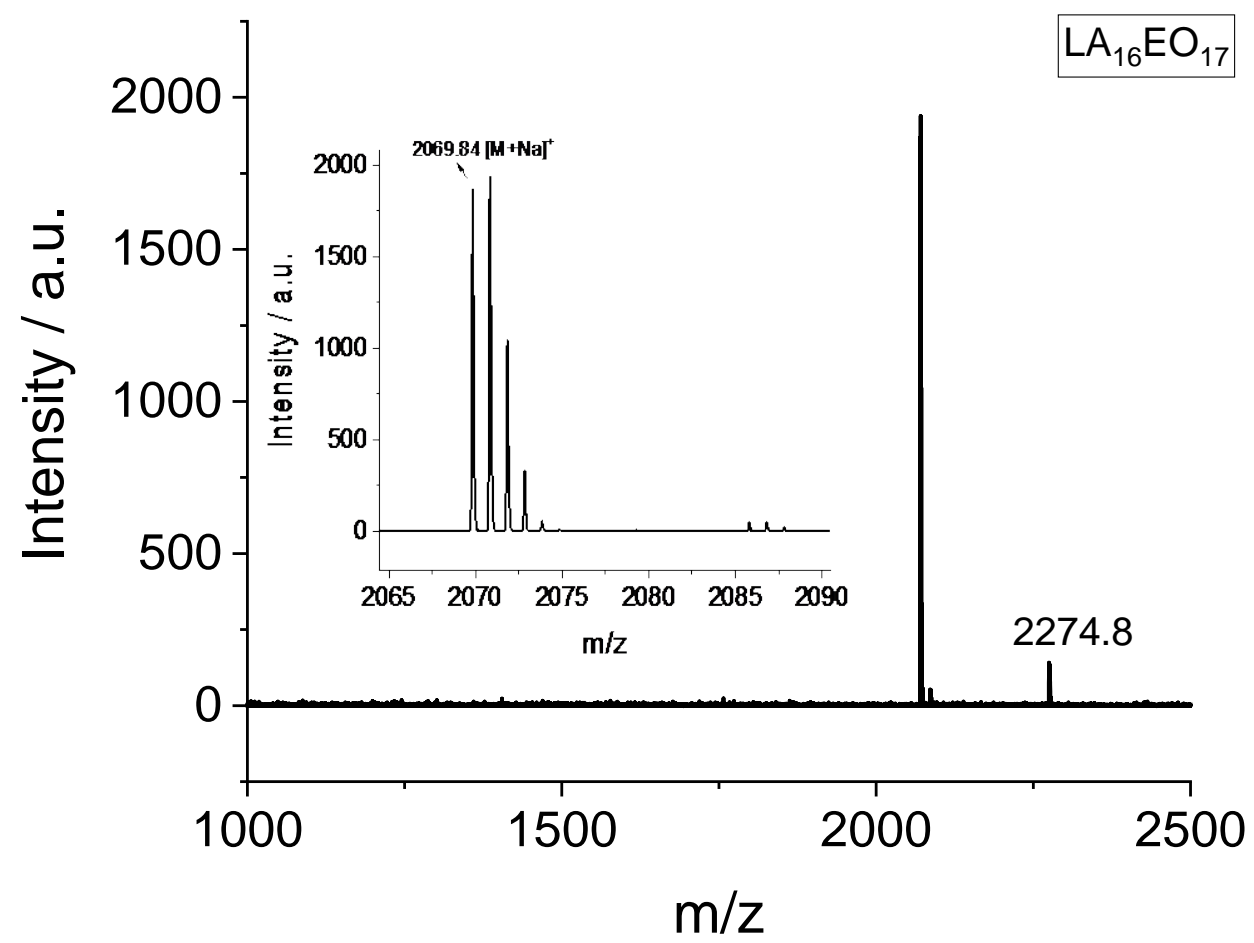

Note: The peak at $m / z=2274.8$ is observed in the CHCA matrix but was not present in the DCTB matrix.

Figure S4b: ${ }^{1} \mathrm{H}$ NMR of $\mathrm{LLA}_{16} \mathrm{EO}_{17}$ :

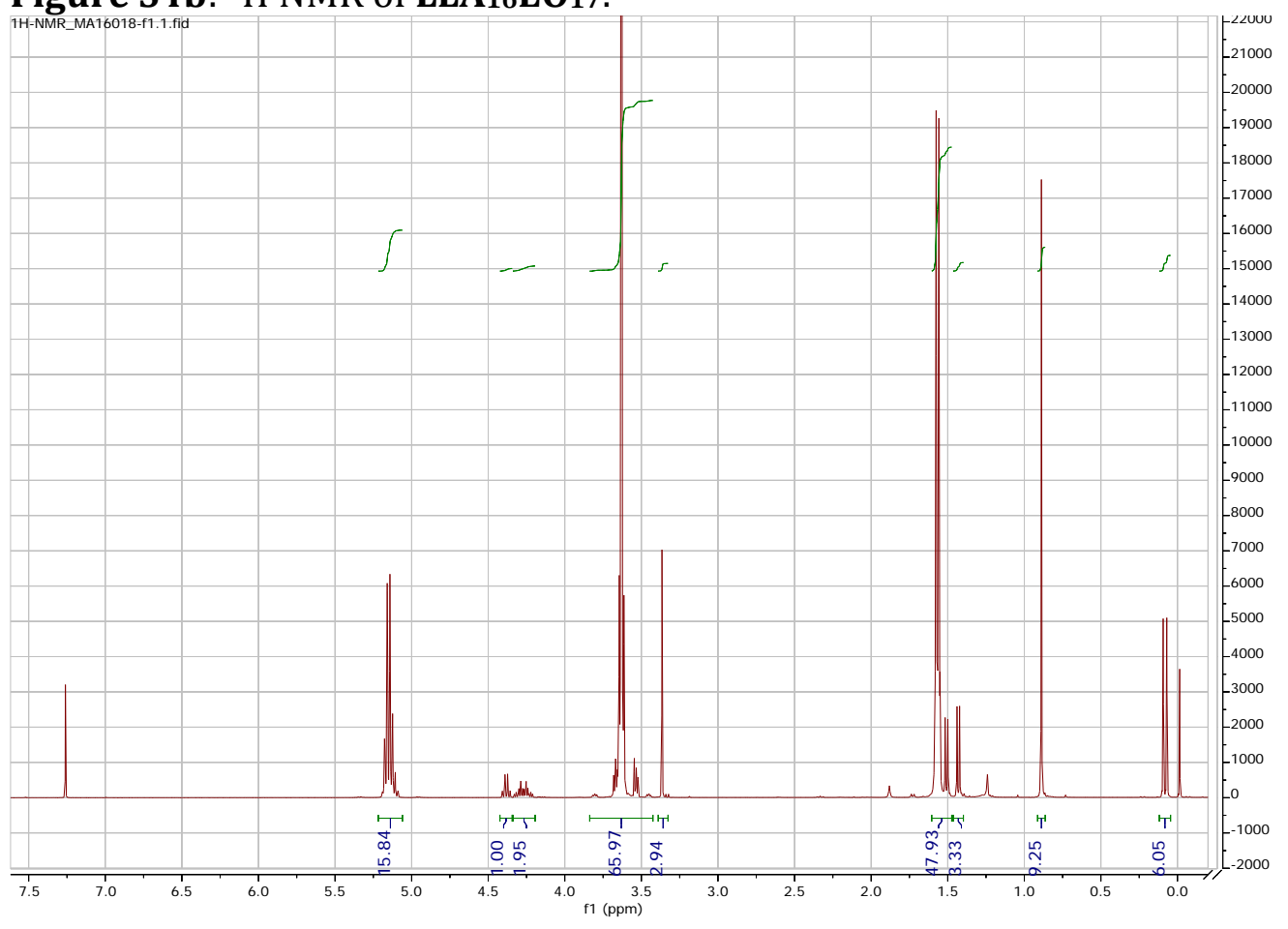


Figure S4c: ${ }^{13}$ C NMR of LLA16EO17:

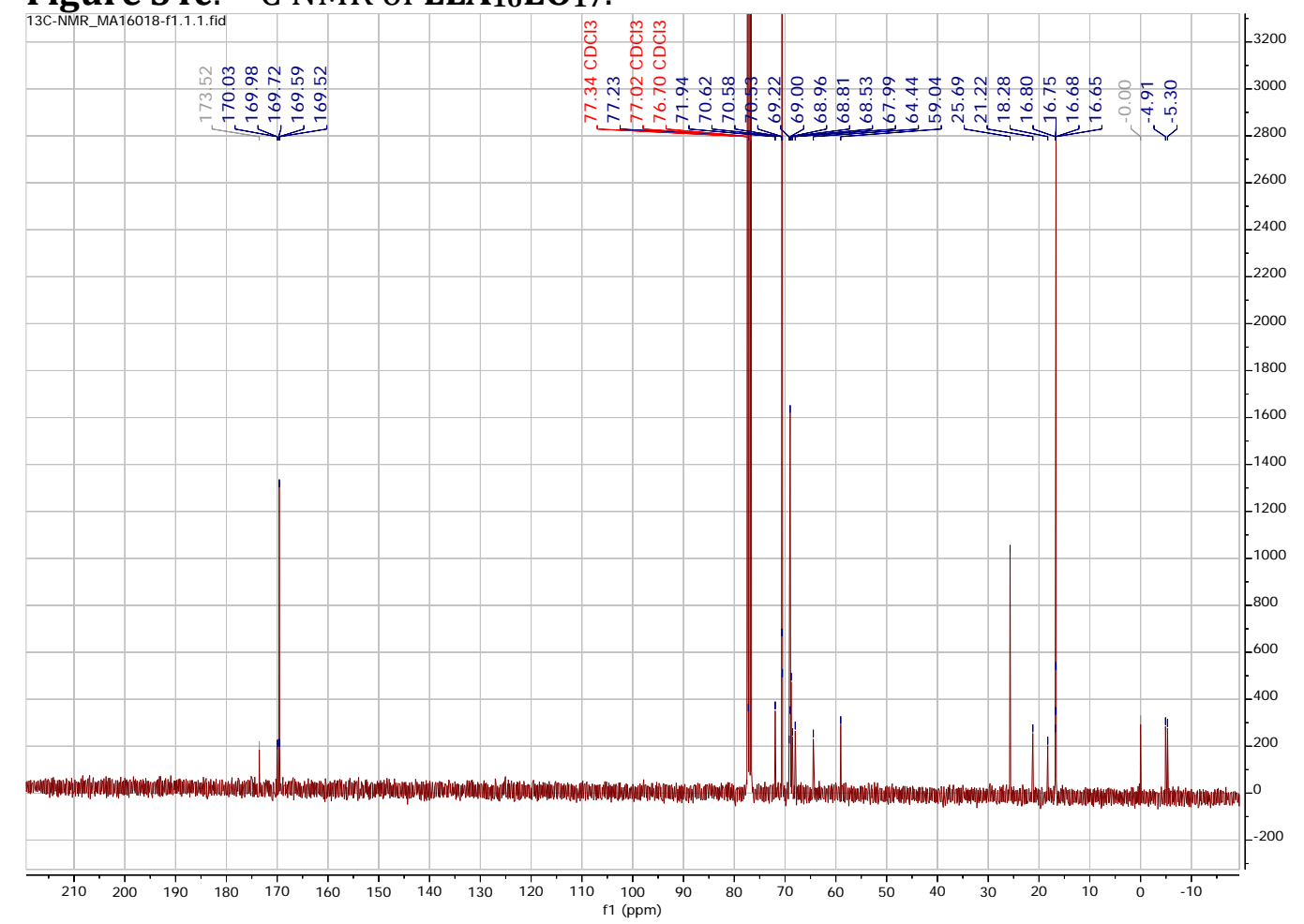

Figure S4d: HSQC NMR of LLA16EO17:

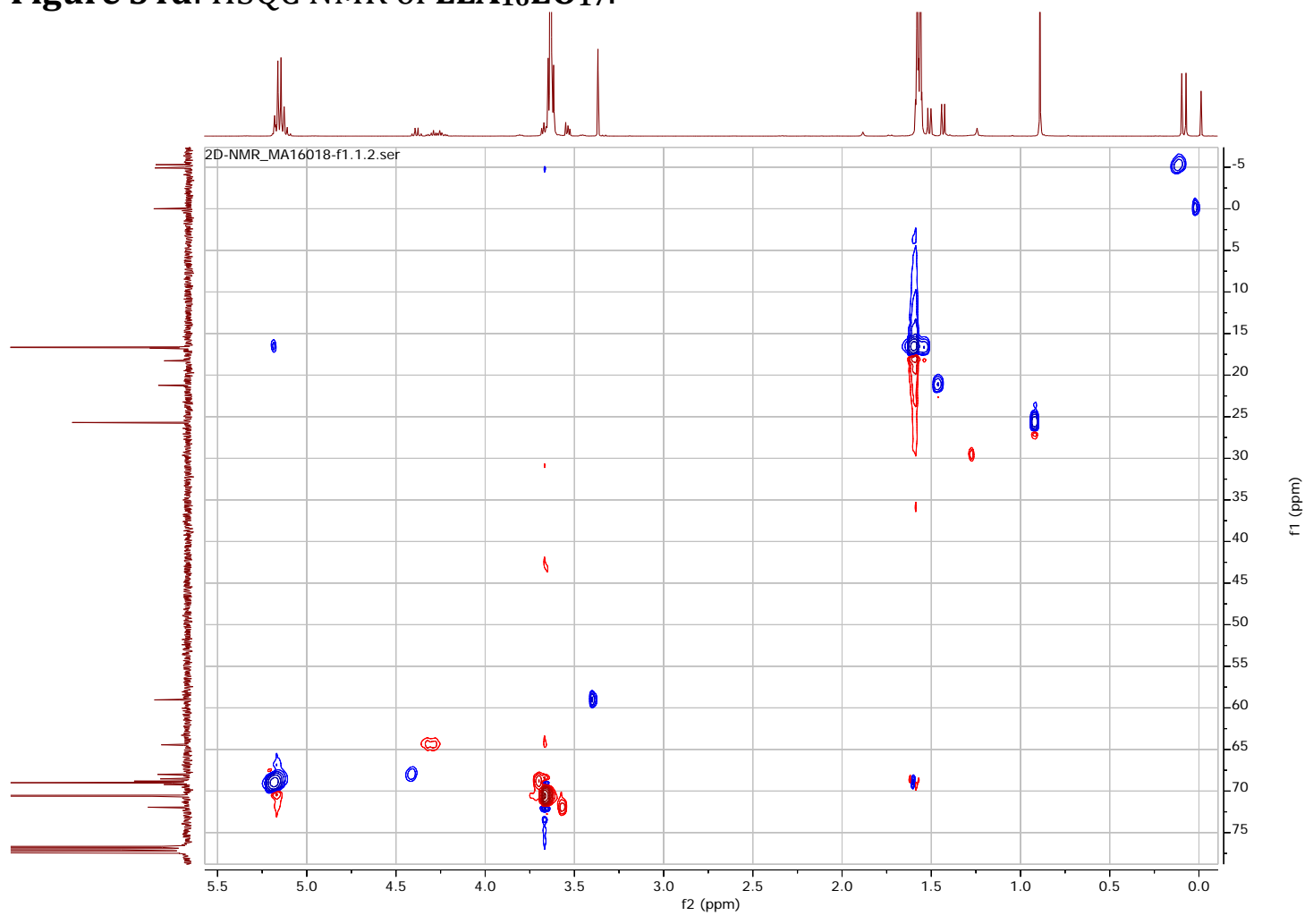


Figure S5a: Maldi-ToF-MS of LLA16EO48:

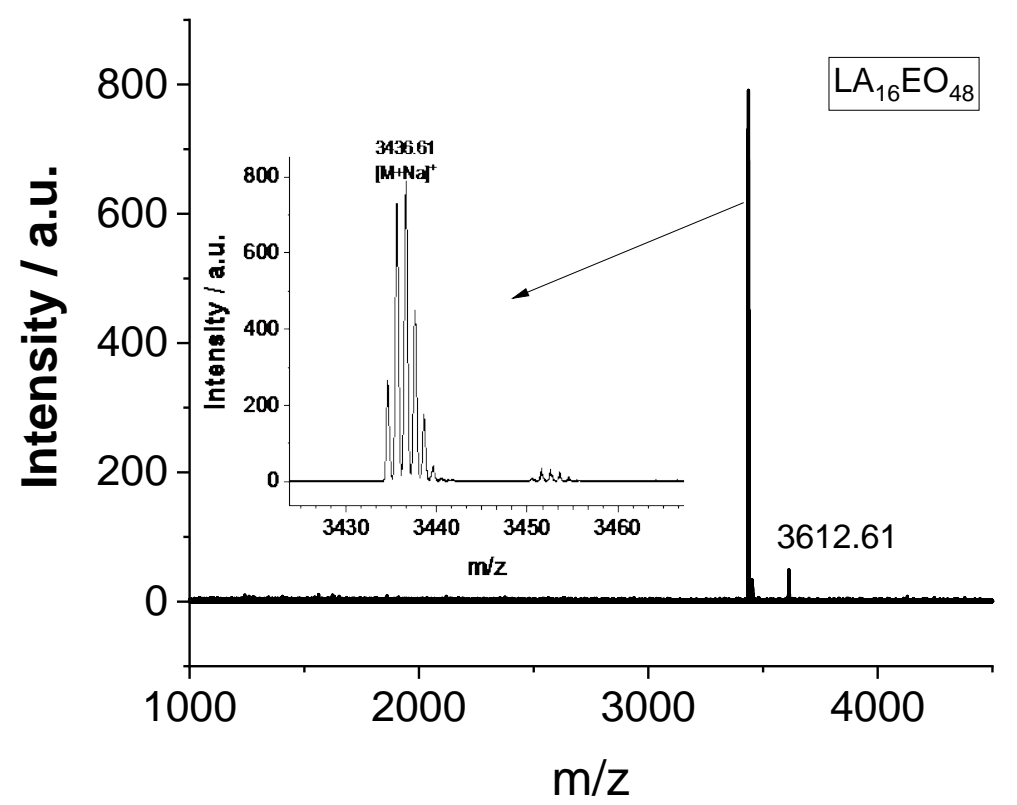

Note: the peak at $m / z=3612.61$ is observed in the CHCA matrix but is not present in the DCTB matrix

Figure S5b: ${ }^{1} \mathrm{H}$ NMR of $\mathrm{LLA}_{16} \mathrm{EO}_{48}$ :

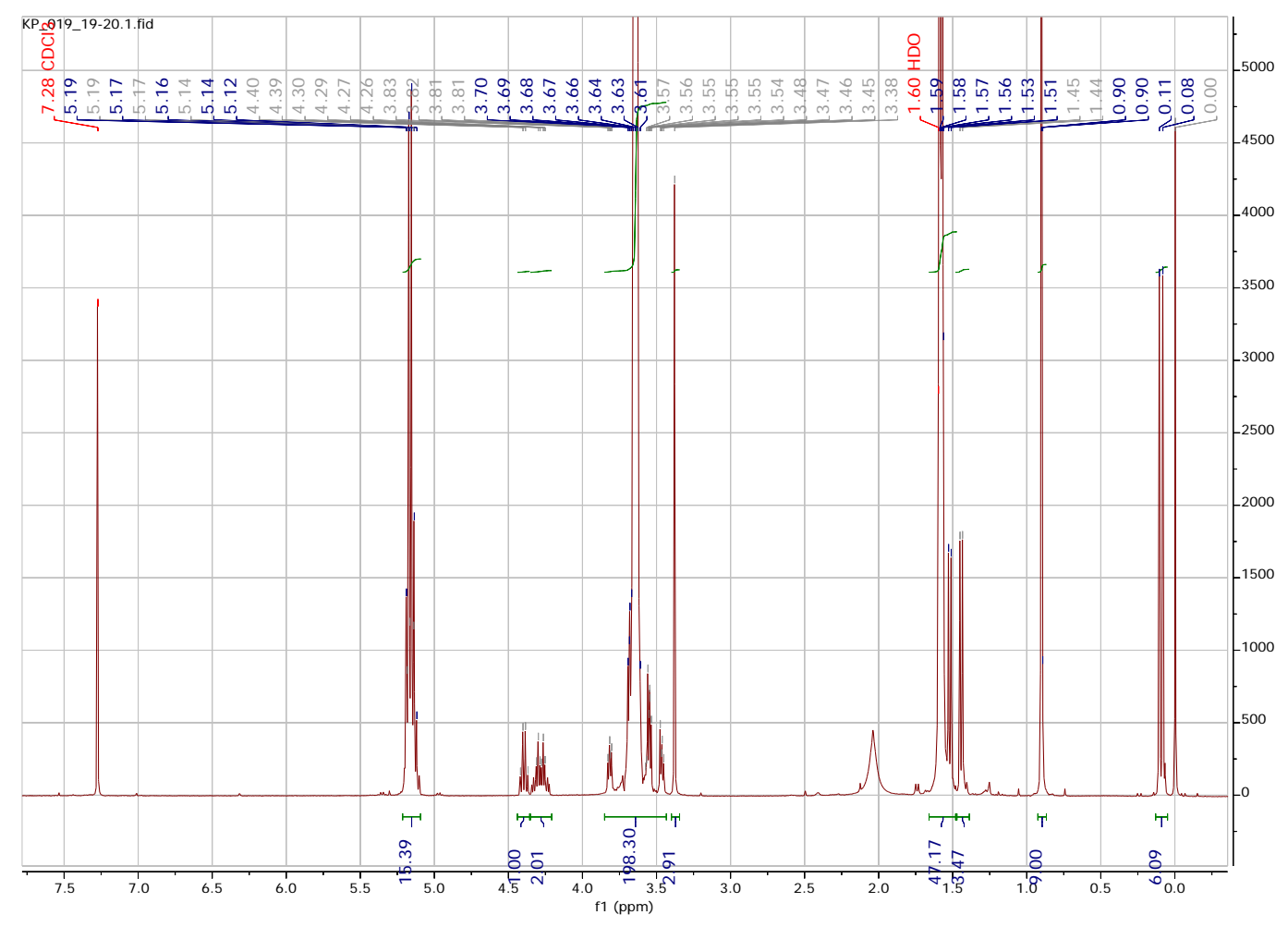


Figure S5c: ${ }^{13} \mathrm{C}$ NMR of LLA16EO48:

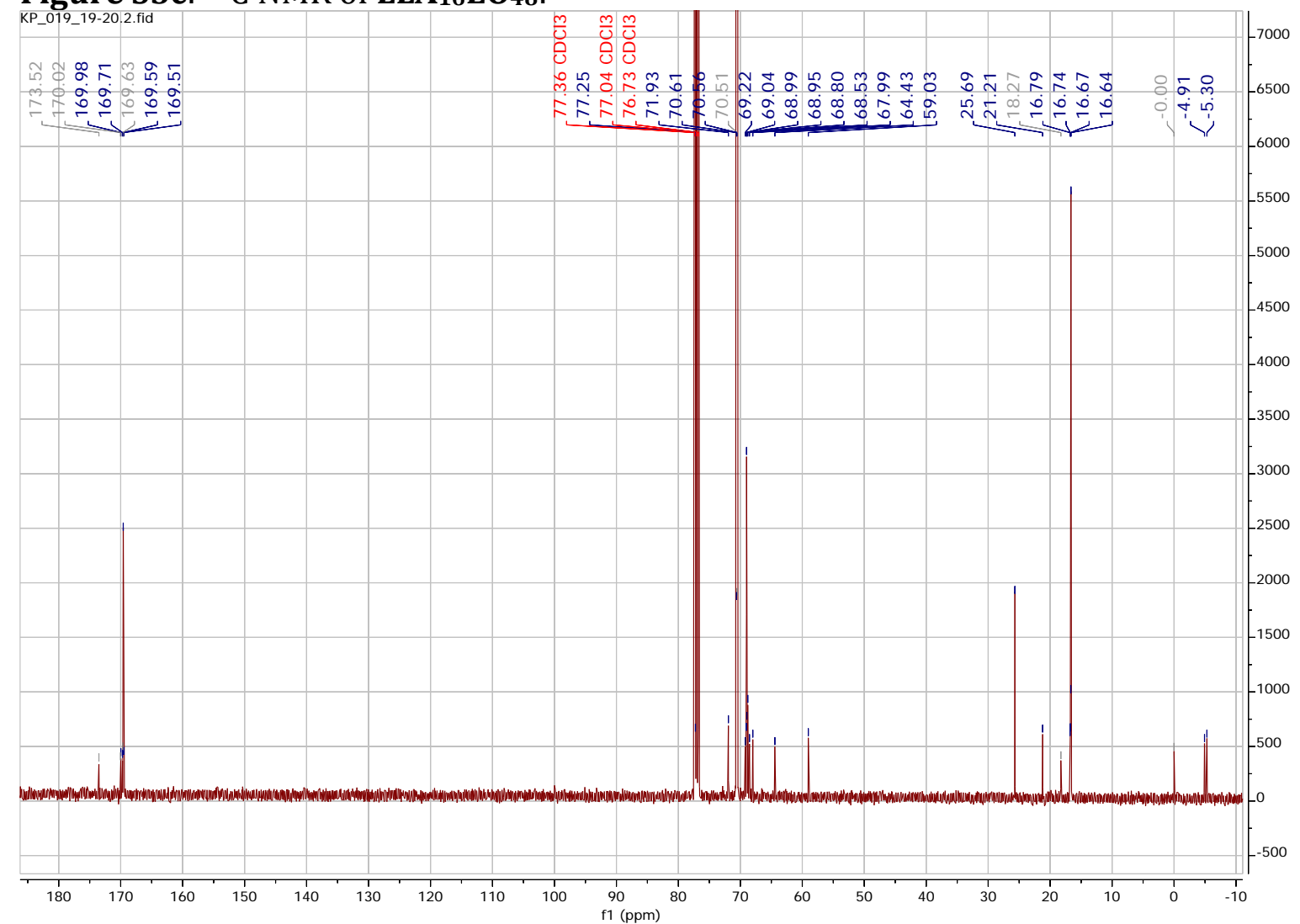


Figure S5d: HSQC NMR of LLA16EO48:

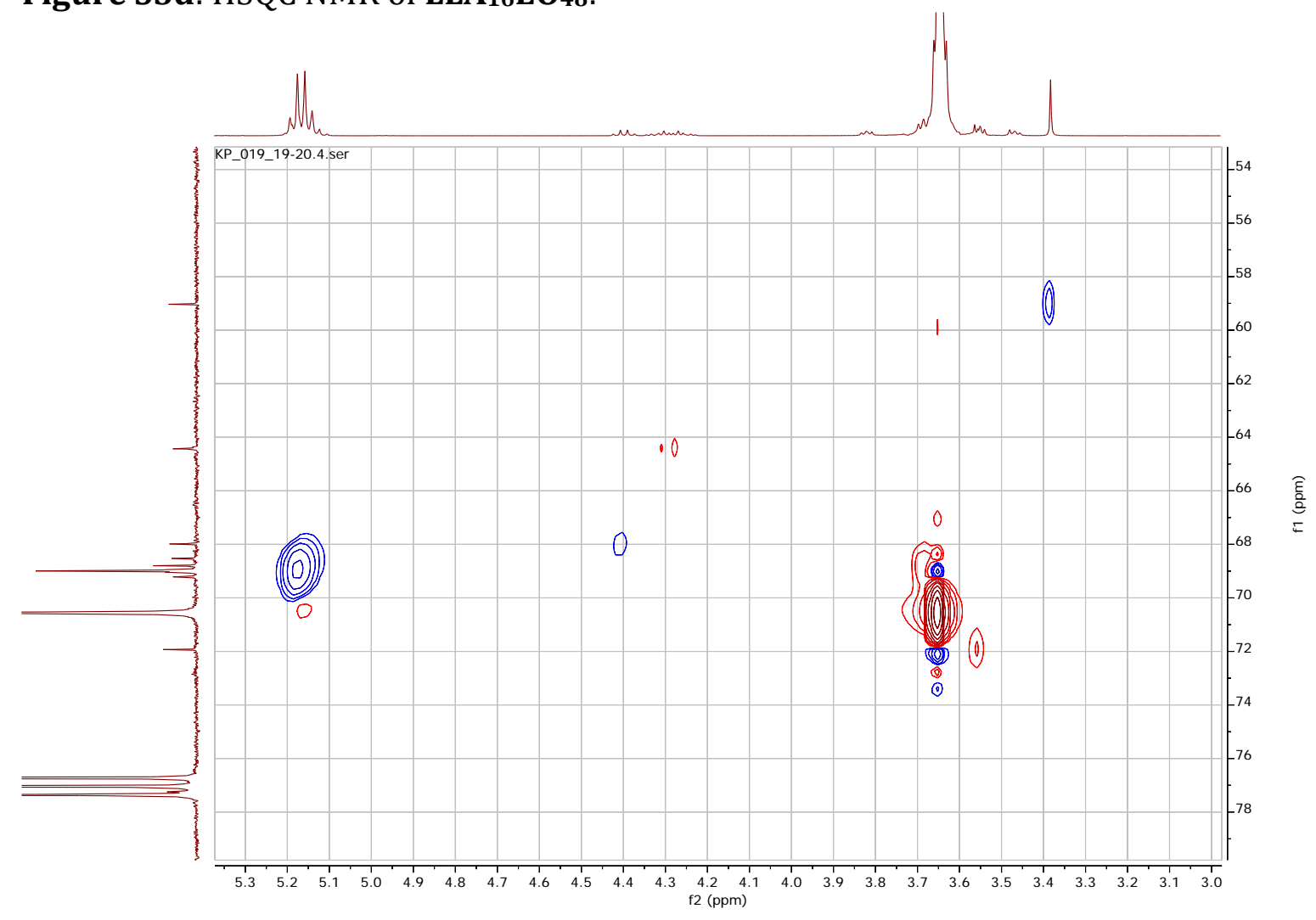

Figure S5e: gCOSY NMR of LLA16EO $\mathbf{L A}_{48}$

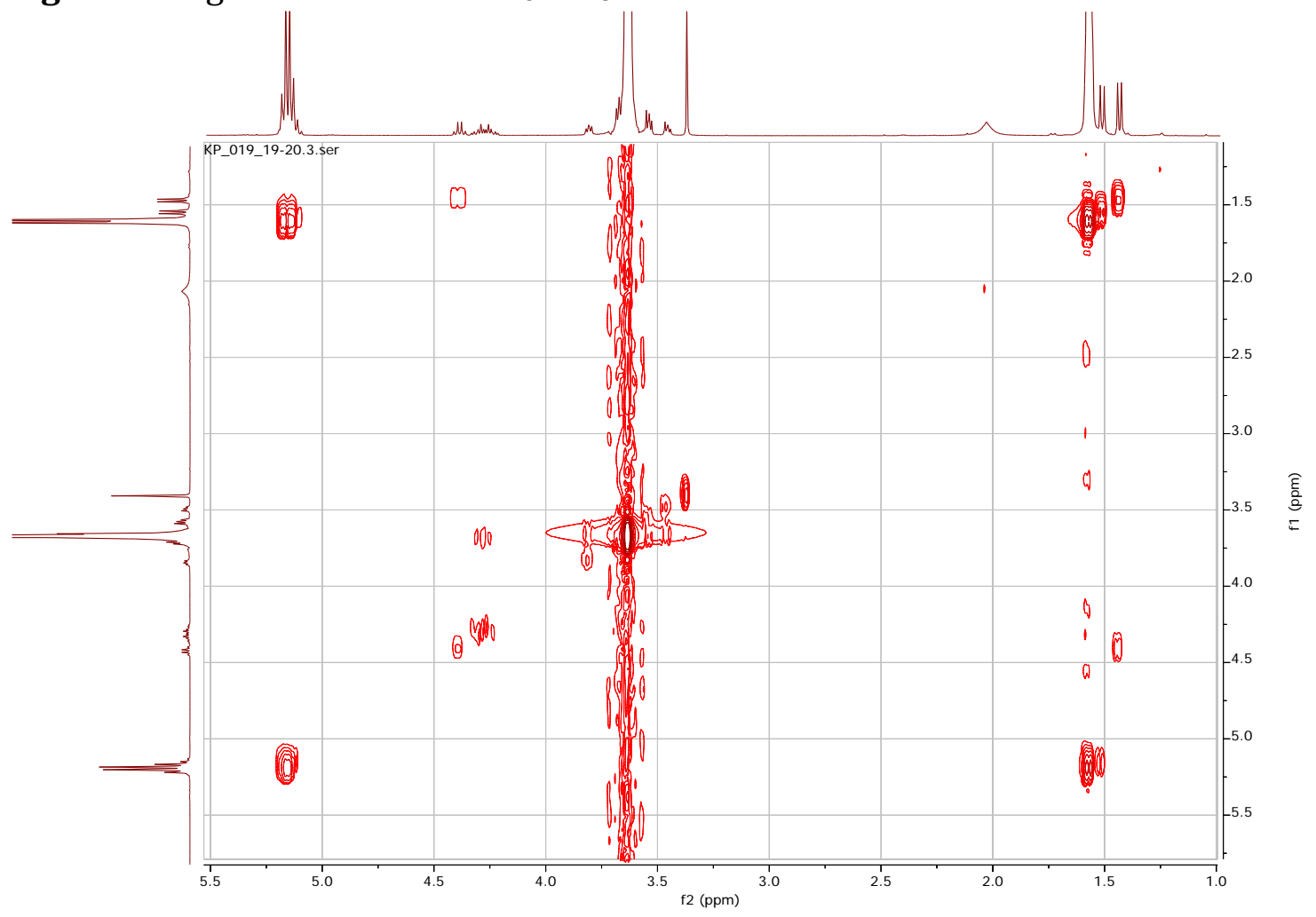


Figure S6a: MALDI-ToF of HO-LLA8EO17:

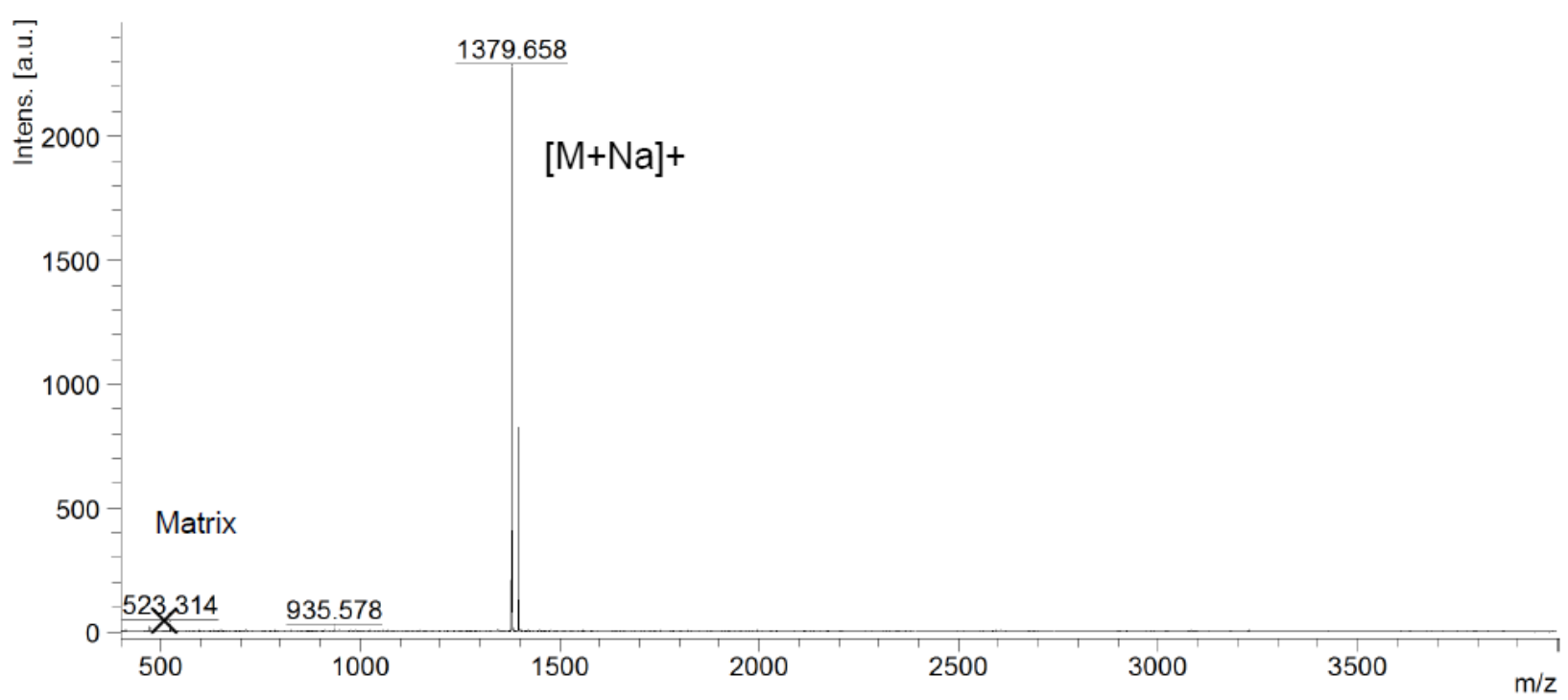

Figure S6b: ${ }^{1} \mathrm{H}-\mathrm{NMR}$ of HO-LLA8EO ${ }_{17}$ :

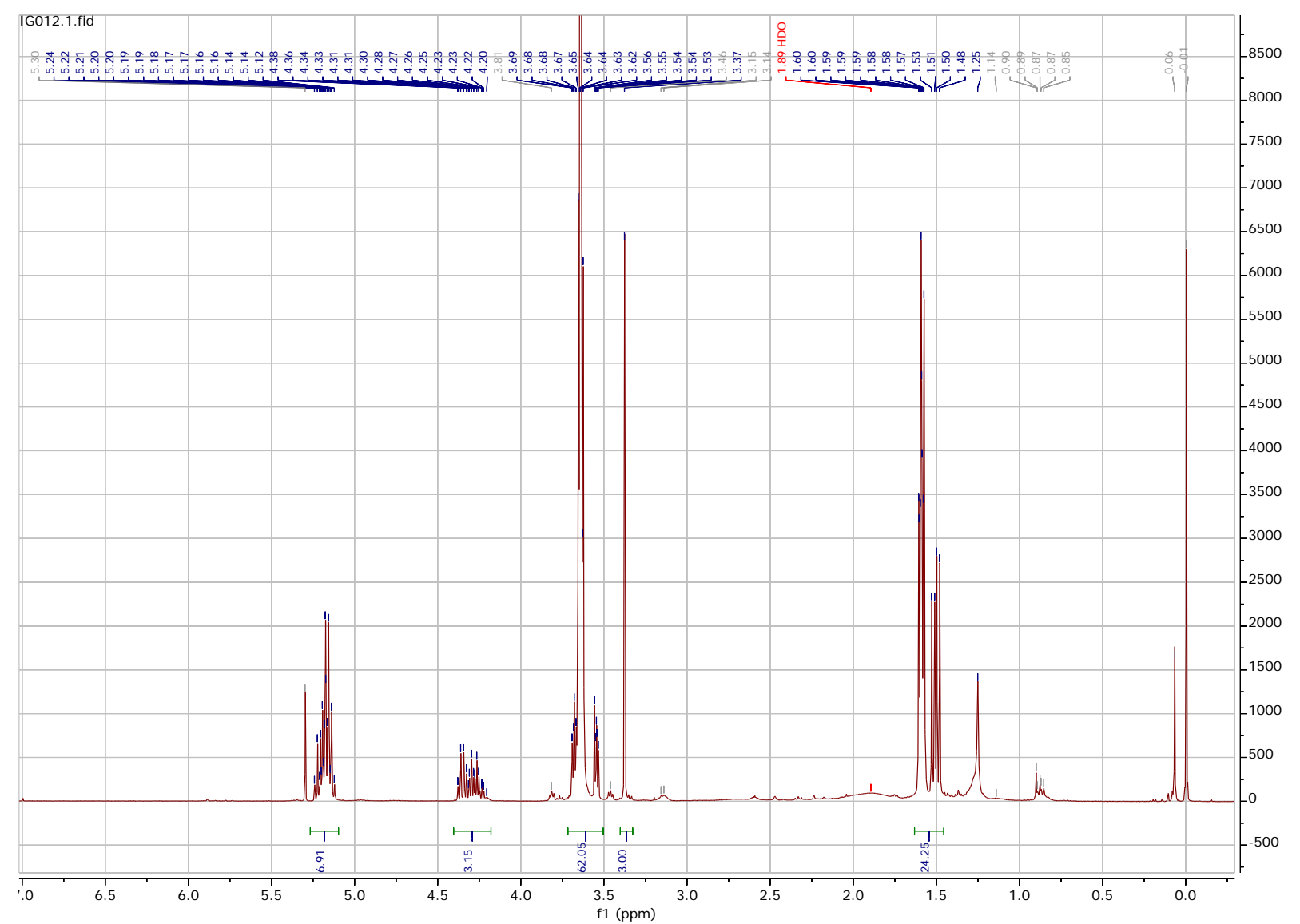


Figure S7a: MALDI-ToF of TBDMS-LLA16-COOH:

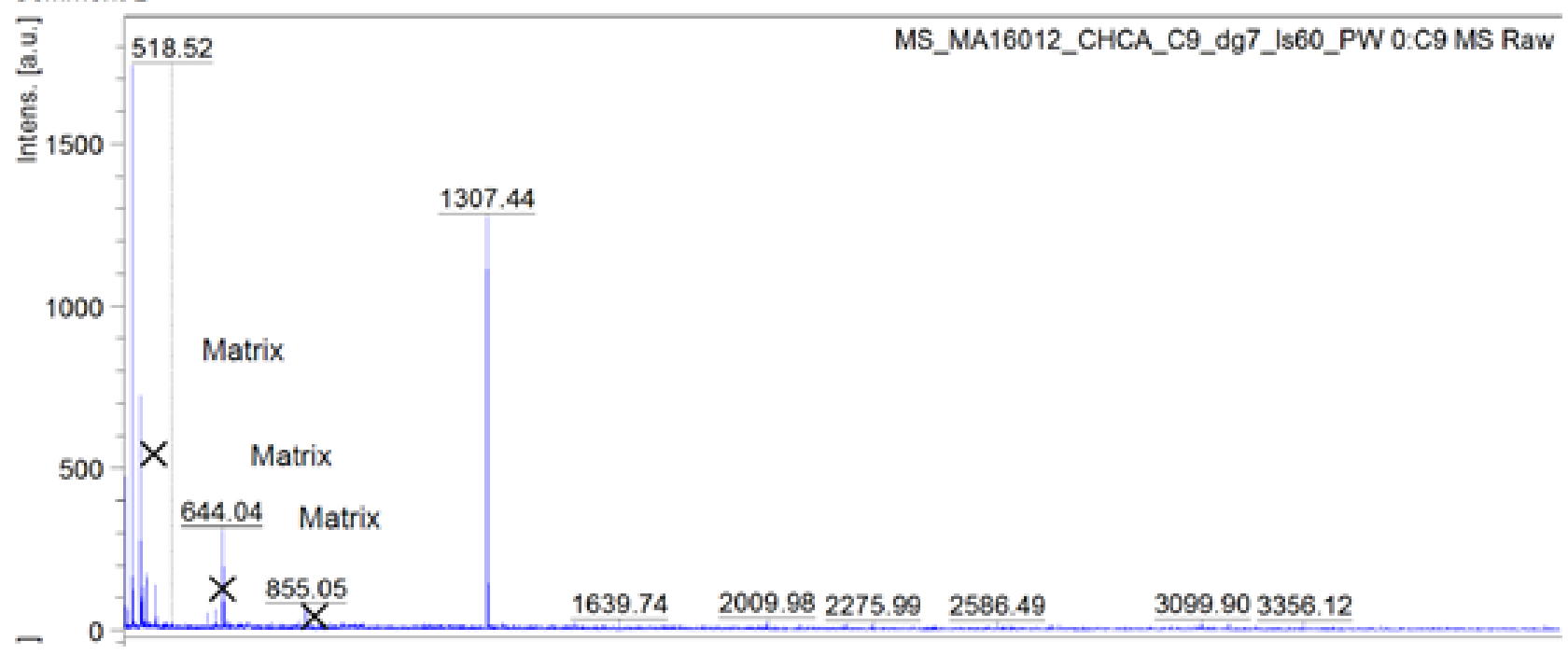

Figure S7b: ${ }^{1} \mathrm{H}-\mathrm{NMR}$ of TBDMS-LLA16-COOH:

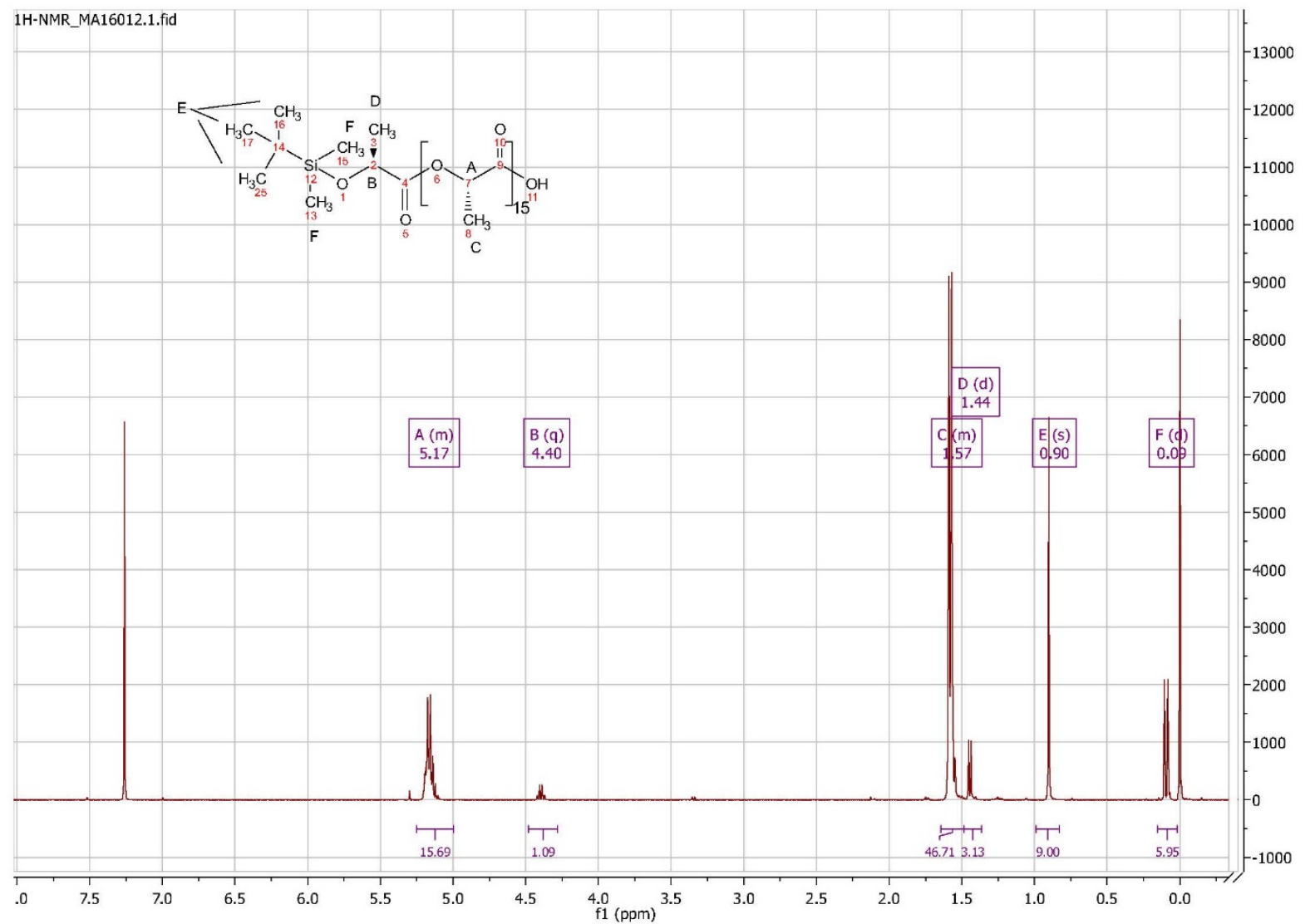




\section{Degradation study of discrete lactic acid-based oligomers}

Differences in stability were tested using 3 precursors; the double protected TBDMS-LLA8-Bn, and the single protected HO-LLA8- $\mathrm{Bn}^{1}$ and TBDMS-LLAs-COOH. These compounds were incubated in a mixture of acetonitrile/phosphate buffer $\mathrm{pH} 7.7(40 / 60)$ at $37^{\circ} \mathrm{C}$ and the degradation was followed by LC-MS. The three 8-mers eluted between 6-9 minutes (TBDMSLLA8-Bn at $8.9 \mathrm{~min}, \mathrm{HO}-\mathrm{LLA}_{8}-\mathrm{Bn}$ at $6 \mathrm{~min}$ and TBDMS-LLA8-COOH at $7.3 \mathrm{~min}$ ) (Figure S8). Upon degradation a series of well-defined peaks with shorter elution times are observed. For HO-LLA8$\mathrm{Bn}$ the major peaks correspond to even number degradation products of HO-LLA8-Bn upon the loss of lactoyllactate upon backbiting: HO-LLA6-Bn, HO-LLA4-Bn, and HO-LLA2-Bn. Very minor peaks corresponding to odd number degradation products from random hydrolysis are visible as well (HO-LLA7-Bn, HO-LLA5-Bn, and HO-LLA3-Bn). For the other two oligomers the appearing peaks corresponded to degradation products from random hydrolysis. Peaks marked in green are TBDMS protected degradation products, whereas the peaks marked in blue correspond to degradation products with a free hydroxyl group.
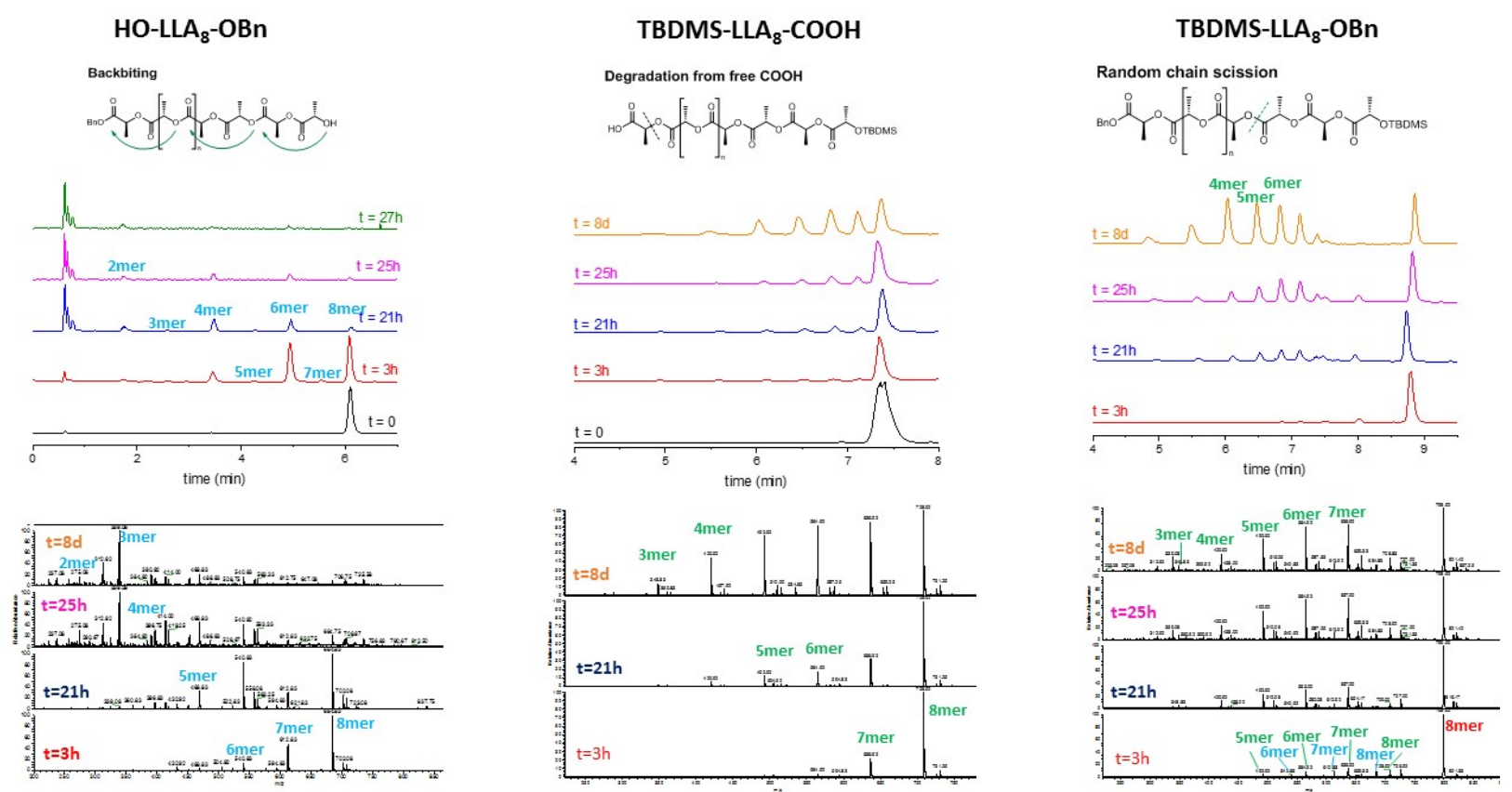

Figure S8: LC-MS results of degradation studies. Top: Total ion count (TIC) traces of HO-LLA8OBn, TBDMS-LLA8-COOH, TBDMS-LLA8-OBn at different time points. Bottom: Detected masses during the run integrated over the whole spectrum.

Oligomers of lactic acid comprising a free hydroxyl group degrade fast through backbiting: an intramolecular attack of the terminal-hydroxyl on the penultimate ester bond, leading to loss of lactoyllactate, and stepwise chain end scission. Significant degradation is already visible after 3 hours and the oligomer is fully degraded after 25 hours. Oligomers of lactic acid with a TBDMS protected hydroxyl group degrade slower through random chain scission. Here, for both, the double and single protected TBDMS-LLA8-Bn and TBDMS-LLA8-COOH, even after 8 days the main component of the sample is the non-degraded 8-mer. When the carboxylic acid is deprotected, the chain scission is less random and preferably occurs at the $\mathrm{COOH}$ end of the oligomer. 


\section{Stability study of alcohol-terminated and TBDMS-terminated BCOs}

Similar results were achieved for the final BCOs incubated at $37^{\circ} \mathrm{C}$ in a $60: 40$ phosphate buffer: $\mathrm{ACN}$ mixture. For $\mathrm{HO}-\mathrm{LLA}_{8} \mathrm{EO}_{17}$, the $\mathrm{BCO}$ with a free hydroxyl group, only peaks corresponding to a dimer $\left(\mathrm{HO}-\mathrm{LLA}_{2} \mathrm{EO}_{17}\right)$, monomer $\left(\mathrm{HO}-\mathrm{LLA}_{1} \mathrm{EO}_{17}\right)$ and just the free $\mathrm{MeO}-\mathrm{EO}_{17}-\mathrm{OH}$ were detected after 3 days. After the same 3 days the main peak for $\mathrm{LLA}_{8} \mathrm{EO}_{17}$, was still the protected $\mathrm{BCO}$, next

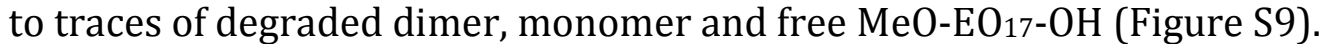

MALDI of $\mathrm{LLA}_{8} \mathrm{EO}_{17}$ (left) and HO-LLA $\mathrm{EO}_{17}$ (right) in THF:
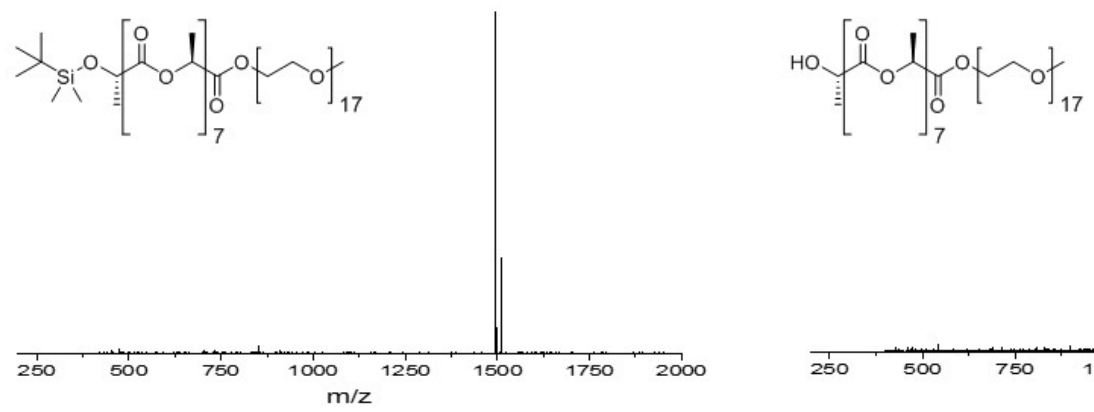

MALDI of $\mathrm{LLA}_{8} \mathrm{EO}_{17}$ (left) and $\mathrm{HO}-\mathrm{LLA}_{8} \mathrm{EO}_{17}$ (right) after incubation for 3 days at $37^{\circ} \mathrm{C}$ in $60: 40$ buffer pH 7: ACN:
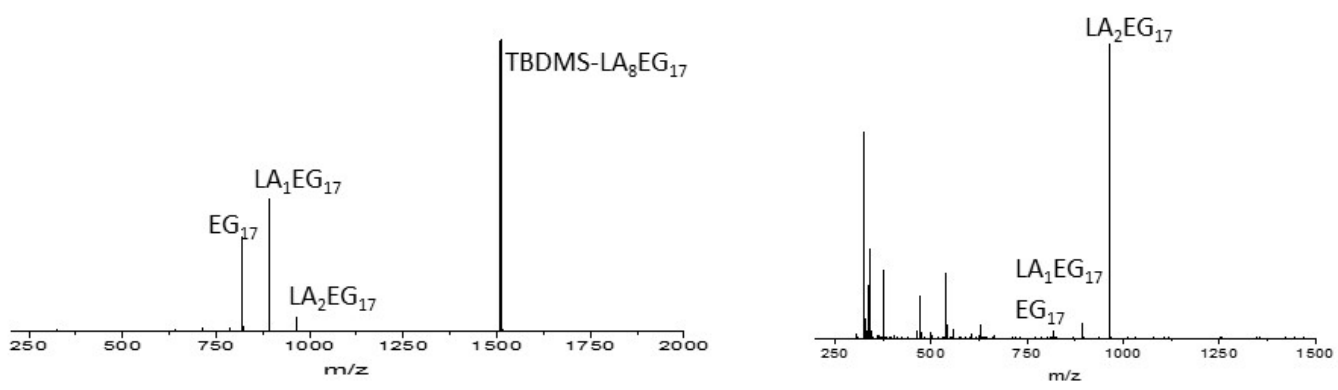

Figure S9: MALDI-ToF spectra of LLA8EO ${ }_{17}$ (left) and HO-LLA8EO ${ }_{17}$ (right). Top: dissolved in THF, bottom: after incubation for 3 days at $37^{\circ} \mathrm{C}$ in a 60:40 buffer pH7: ACN.

To see if the self-assembled BCOs degrade overtime in aqueous solution, the $1 \mathrm{mg} / \mathrm{mL} \mathrm{LLA}_{16} \mathrm{EO}_{17}$ sample $\left([\mathrm{M}+\mathrm{Na}]^{+}=2070.90\right)$ was measured with MALDI-ToF 42 days after preparation. Only two minor peaks of $\mathrm{HO}-\mathrm{LLA}_{16} \mathrm{EO}_{17}$ and $\mathrm{EO}_{17}$ were detected and no further traces of degradation were observed (Figure S10). 


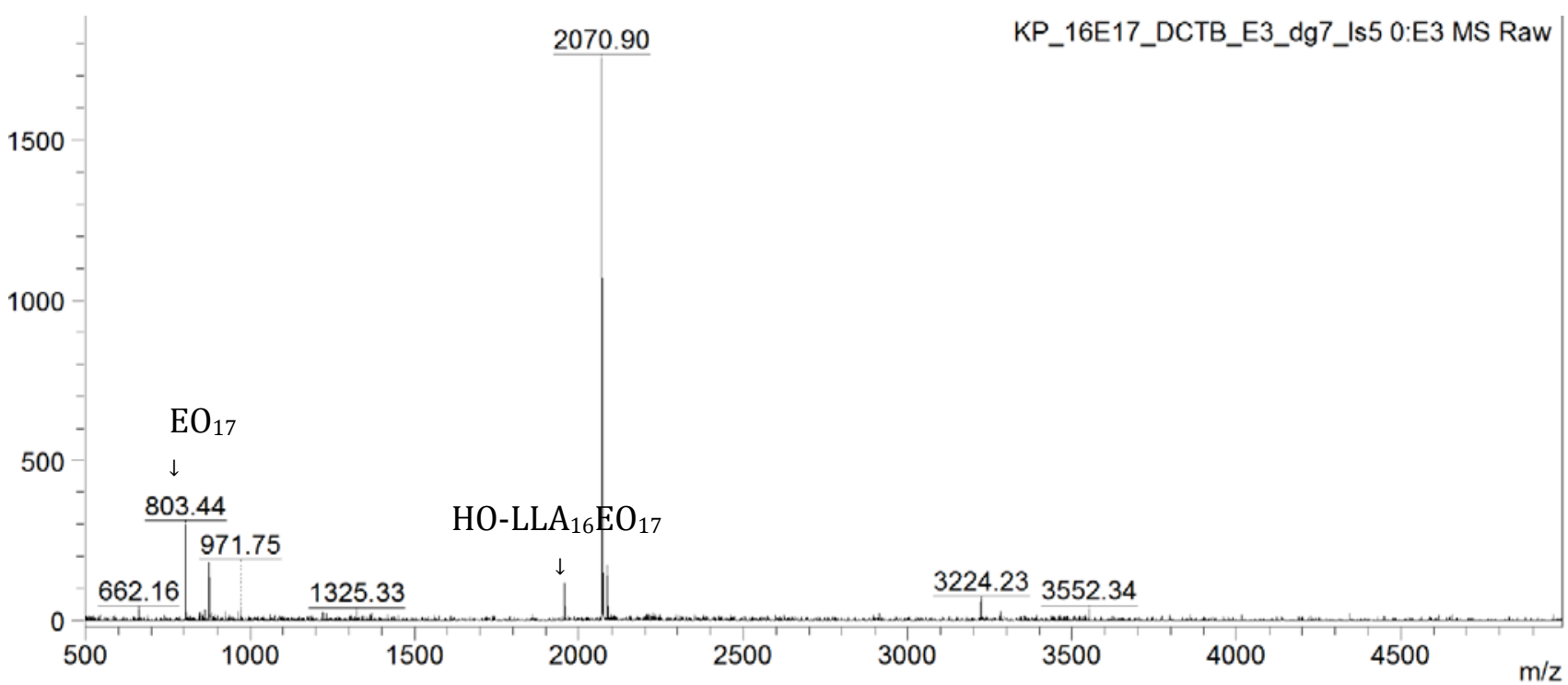

Figure S10: MALDI-ToF spectrum of a 42 days old sample of self-assembled particles of LLA16EO17.

A similar study was conducted for investigating $\mathrm{LLA}_{16} \mathrm{EO}_{48}$. A $1 \mathrm{mg} / \mathrm{mL} \mathrm{LLA}_{16} \mathrm{EO}_{48}$ sample $\left([\mathrm{M}+\mathrm{Na}]^{+}=3436.86\right)$ was measured with MALDI-ToF 52 days after preparation and no degradation was observed (Figure S11).

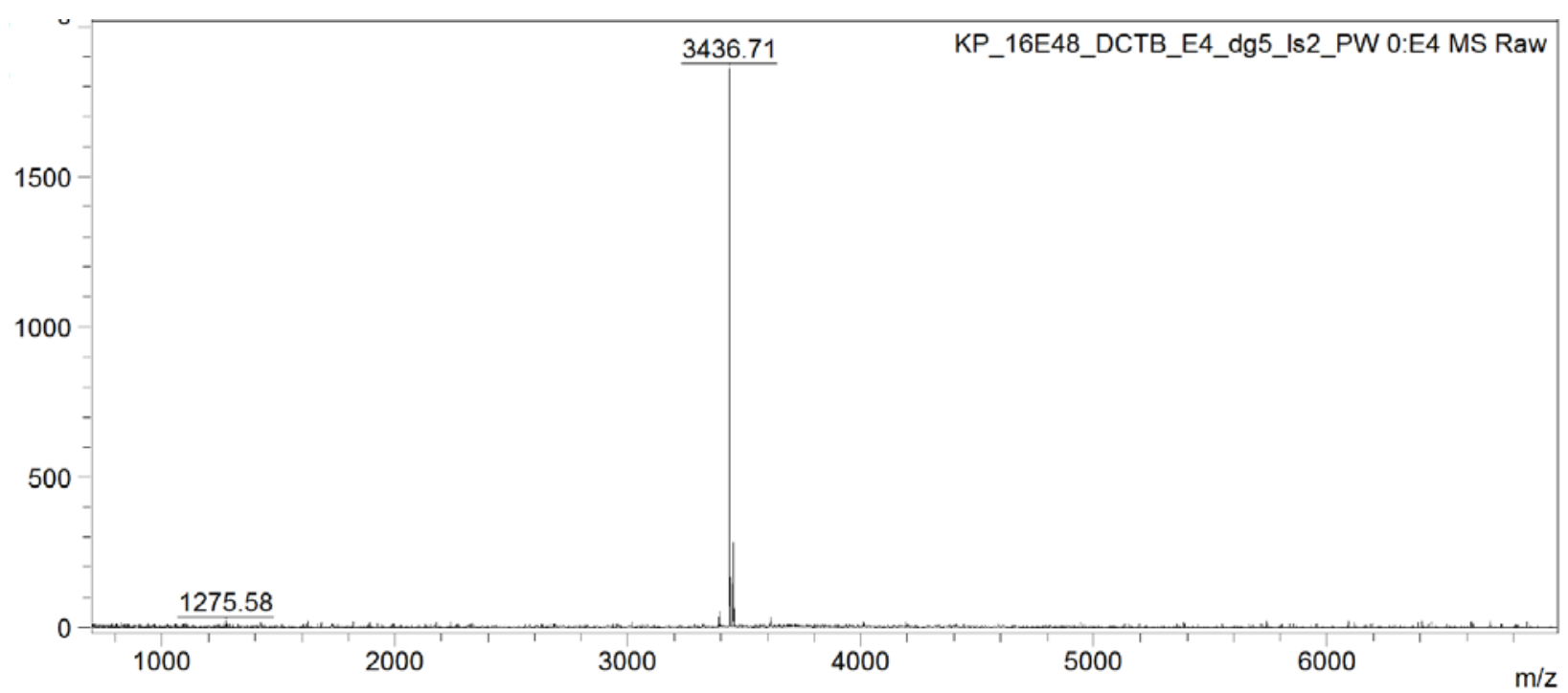

Figure S11: MALDI-ToF spectrum of 52days old self-assembled particles of LLA16 $\mathrm{EO}_{48}$. 


\section{Bulk properties of BCOs}
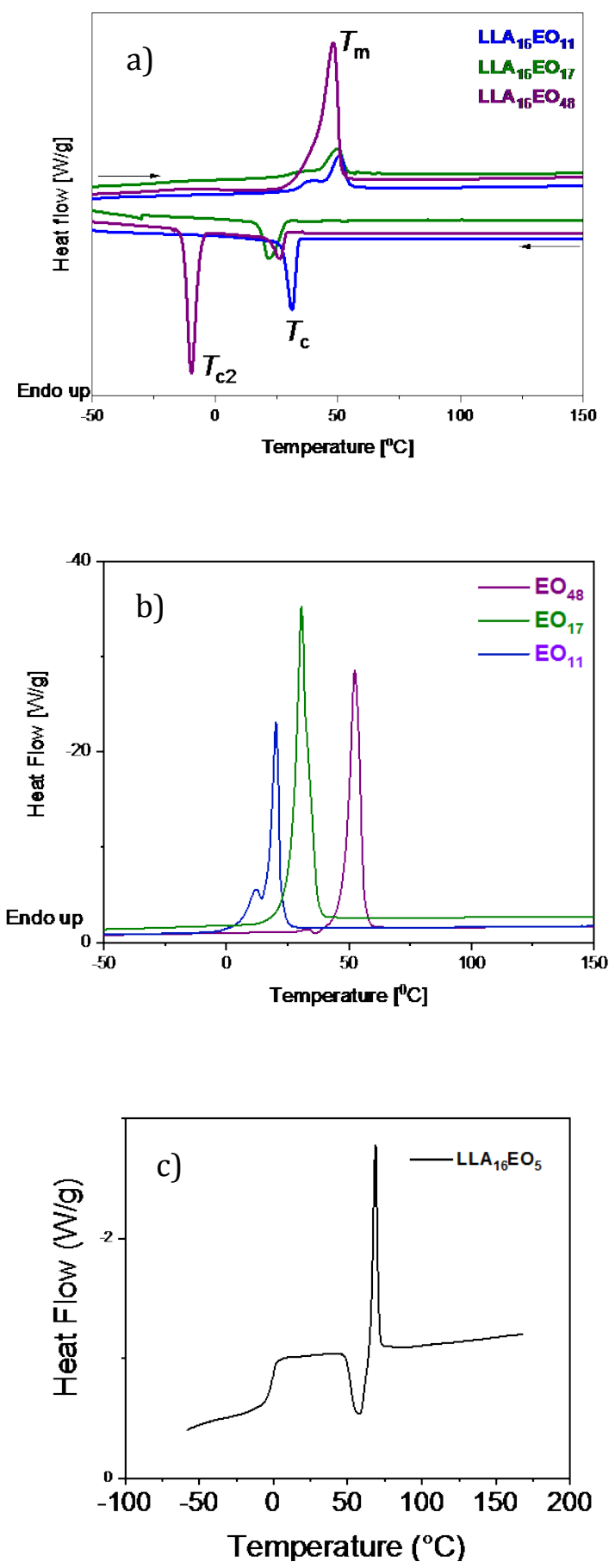

Figure S12: a) DSC traces (first cooling and second heating run) for $\mathrm{LLA}_{16} \mathrm{EO}_{11}$ (blue), $\mathrm{LLA}_{16} \mathrm{EO}_{17}$ (green) and LLA $16 \mathrm{EO}_{48}$ (purple). Method: heating from RT to $150{ }^{\circ} \mathrm{C}$, cooling to $-85^{\circ} \mathrm{C}$ followed by heating to $160^{\circ} \mathrm{C}\left(10^{\circ} \mathrm{C} / \mathrm{min}\right) . \mathrm{T}_{\mathrm{m}}=$ melting temperature, $\mathrm{T}_{\mathrm{c}}=$ crystallization temperature $\left.\mathrm{b}\right) \mathrm{DSC}$ traces (second heating run, heating from RT to $150{ }^{\circ} \mathrm{C}$, cooling to $-85^{\circ} \mathrm{C}$ followed by heating to $\left.160{ }^{\circ} \mathrm{C}\left(10^{\circ} \mathrm{C} / \mathrm{min}\right)\right)$ for $\mathrm{EO}_{11}, \mathrm{EO}_{17}$ and $\mathrm{EO}_{48}$. c) DSC trace of LLA16E05 (second heating run, heating from RT to $150^{\circ} \mathrm{C}$, cooling to $-85^{\circ} \mathrm{C}$ followed by heating to $\left.160^{\circ} \mathrm{C}\left(10{ }^{\circ} \mathrm{C} / \mathrm{min}\right)\right)$ 
Table S1: Melting and crystallization temperatures and corresponding enthalpies of BCOs and EO / LLA oligomersa

\begin{tabular}{|l|l|l|l|l|}
\hline Compound & $\begin{array}{l}\boldsymbol{T}_{\mathbf{m}} \\
{\left[{ }^{\circ} \mathrm{C}\right]}\end{array}$ & $\begin{array}{l}\Delta \boldsymbol{H}_{\mathrm{Tm}} \\
{[\mathbf{k J} / \mathbf{m o l}]}\end{array}$ & $\begin{array}{l}\boldsymbol{T}_{\mathbf{c}} \\
{\left[{ }^{\circ} \mathbf{C}\right]}\end{array}$ & $\begin{array}{l}\Delta \boldsymbol{H}_{\text {Tc }} \\
{[\mathbf{k J} / \mathbf{m o l}]}\end{array}$ \\
\hline $\mathrm{LLA}_{16}-\mathrm{EO}_{5}$ & 68.6 & 17.5 & none $^{\mathrm{b}}$ & none $^{\mathrm{b}}$ \\
\hline $\mathrm{LLA}_{16}-\mathrm{EO}_{11}$ & 50 & 28.4 & 22 & 32 \\
\hline $\mathrm{LLA}_{16}-\mathrm{EO}_{17}$ & 51 & 48.1 & 32 & 43 \\
\hline $\mathrm{LLA}_{16}-\mathrm{EO}_{48}$ & 48 & 295.6 & $-10 ; 27$ & $152 ; 28$ \\
\hline $\mathrm{EO}_{11}$ & 20.2 & 75.1 & 1.1 & 68.5 \\
\hline $\mathrm{EO}_{17}$ & 30.5 & 130.6 & 13.5 & 123.9 \\
\hline $\mathrm{EO}_{48}$ & 52.3 & 414.5 & $22.4 ; 16.9$ & 372.2 \\
\hline $\mathrm{LLA}_{16}{ }^{\mathrm{c}}$ & 89.6 & 70.9 & - & - \\
\hline
\end{tabular}

a All data from second heating and first cooling; b compound does not show crystallization during cooling; $\mathrm{c}$ data from ref 2

We measured DSC of EO 48 which shows a melting point of $52.3^{\circ} \mathrm{C}$ and a crystallization of $16.9^{\circ} \mathrm{C}$. The enthalpies are very high, which explains the much higher enthalpy of the melting transition in $\mathrm{LLA}_{16} \mathrm{EO}_{48}$ compared to the two other BCOs. In the cooling run, a second transition $\mathrm{T}_{\mathrm{c} 2}$ is observed for LLA16 $\mathrm{EO}_{48}$ which is attributed to the crystallization of the $\mathrm{EO}_{48}$ block $\left(\mathrm{T}_{\text {cryst }}\left(\mathrm{EO}_{48}\right)=\right.$ $\left.22.4^{\circ} \mathrm{C}\right)$

X-ray studies previously done on pEO comprising systems by Kyritsis and coworkers ${ }^{3}$ show that the WAXS region has sharp reflections at $q=13.6,16.5$ and $18.5 \mathrm{~nm}^{-1}$ which were attributed to the crystalline order between the PEO chains. This work also showed that crystalline order actually persist well above $T_{\mathrm{m}}$ when all lamellar ordering is gone. In our case, we find sharp reflections at identical positions for $\mathrm{LLA}_{16} \mathrm{EO}_{48}$ in the WAXS region $\left(q=13.9,16.6\right.$ and $\left.18.5 \mathrm{~nm}^{-1}\right)$, corroborating that at RT we have crystalline order between the $\mathrm{EO}_{48}$ chains as well. However, the LLA part has lost the sharp reflections in the WAXS region (the sharp peak around $10 \mathrm{~nm}^{-1}$ disappeared) which may cause the loss of lamellar order in de MAXS region. Similar to what was observed by Kyristis et al, some crystalline order between pEO chains persist but the lamellar order is gone in $\mathrm{LLA}_{16} \mathrm{EO}_{48}$. 


\section{MicroDSC and TIRF measurements of $\mathrm{LLA}_{16} \mathrm{EO}_{11}$}

The particle size obtained for $\mathrm{LLA}_{16} \mathrm{EO}_{11}$, which is predicted to form bilayers/vesicles, is large enough for visualization with microscopy. CryoTEM was unsuccessful for this sample, but the particles could be visualized using TIRF microscope after addition of Nile Red (final concentration $=1 \mu \mathrm{M})$. Spherical particles which did not interact with each other were visible (Figure S13 left). In addition, microDSC measurements were performed of $\mathrm{LLA}_{16} \mathrm{EO}_{11}$ selfassembled at $5 \mathrm{mg} / \mathrm{mL}$ with $10 \% \mathrm{THF}$ in water to investigate if the LLA part is able to crystallise. The heating run showed a clear melting transition at $46{ }^{\circ} \mathrm{C}$, the cooling run also showed a crystallisation peak at $32^{\circ} \mathrm{C}$ (Figure S13 right).
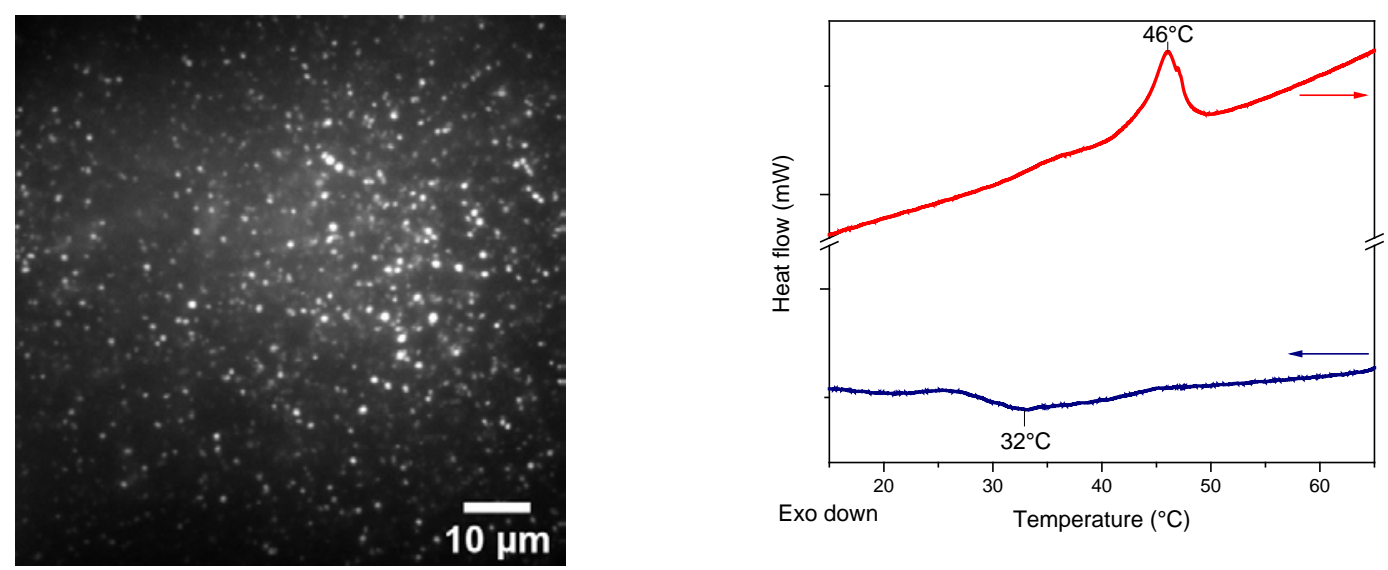

Figure S13: left: TIRF microscopy image of a solution of $\mathrm{LLA}_{16} \mathrm{EO}_{11}$ incubated with Nile Red (final concentration $1 \mu \mathrm{M}$ ). right: microDSC of LLA16EO $\mathrm{E}_{11}$ self-assembled at $5 \mathrm{mg} / \mathrm{mL}$ in $10 \% \mathrm{THF}$ in water, cooling (blue) and heating (red) run.

\section{Scheutjens-Fleer Self-Consistent Field (SCF) computations}

Setup of the numerical SCF lattice computations

The numerical SCF computations have been performed using lattices containing 100 lattice layers in the gradient direction and different geometries to determine the preferred morphology of the assemblies (spherical, cylindrical and flat geometry to simulate the formation of micelles, cylindrical micelles and vesicles respectively). It was verified the lattice size was sufficient to avoid any lattice confinement effects. As coordination number of the lattice $z=6$ was used for all the geometries. In flat geometry two mirrors are placed at the first and the last lattice layers to ensure periodic boundaries conditions. For spherical and cylindrical geometries a mirror was located at the last lattice layer (the first one corresponds to the center of the lattice).

In the lattice each solvent molecule (W) and the monomers composing the blocks (EO, LA) are modeled such that they occupy a single lattice site. Each lattice site was considered to have a size $l$ corresponding to the size of a LLA monomer $(l=0.45 \mathrm{~nm})$. This value was estimated from

$$
l=\left(\frac{M_{\mathrm{LLA}}}{\rho N_{\mathrm{AV}}}\right)^{\frac{1}{3}},
$$


where $N_{\mathrm{AV}}$ is the Avogadro's number, $M_{\mathrm{LLA}}$ and $\rho$ are respectively the molar mass of the LLA monomer $\left(\approx 72 \mathrm{~g} \cdot \mathrm{mol}^{-1}\right)$ and the density of LLA $\left(\rho \approx 1.3 \mathrm{~g} \cdot \mathrm{cm}^{-3}\right)$.

The value of the interaction parameters $\chi_{\mathrm{EO}-\mathrm{W}}=0.49$ and $\chi_{\mathrm{LA}-\mathrm{W}}=3.5$ have been evaluated from literature data. ${ }^{4,5}$ The value $\chi_{\mathrm{EO}-\mathrm{LA}}=1$ has been chosen as effective value because the presence of hydrogen-bonded water molecules on the PEO chains mediates the interaction between the blocks, resulting in a high inter-block segregation. ${ }^{6}$

Modeling of the self-assembly of monodisperse $\mathrm{LLA}_{16}-\mathrm{EO}_{\mathrm{n}}$ copolymers in water

To determine the preferred morphology in water of LLA16 based block copolymers as a function of the EO block length a set of monodisperse copolymers with composition $\mathrm{LLA}_{16} \mathrm{EO}_{\mathrm{n}}$ have been modeled (Table S1). The length of the PEO block was varied between $n=2$ and $n=50$ with a step of 2 units. At each composition the self-assembly of the copolymer was studied in spherical, cylindrical and flat geometries to determine the preferred morphology of the assemblies. This can be done by comparing the volume fraction of copolymer segments in the bulk ( $\left.\varphi^{\text {bulk}}\right)$ obtained in the different geometries. Such a quantity correspond the concentration of nonassociated copolymer molecules in equilibrium with the self-assembled structure. The geometry in which the copolymer has lowest $\varphi^{\text {bulk }}$ corresponds to the preferred morphology, since the chemical potential of a block copolymer in the self-assembled structure (sas) can be expressed as $^{7}$

$$
\mu_{\mathrm{P}}^{\mathrm{sas}} \approx k_{B} T \ln \left(\varphi^{\text {bulk }}\right) .
$$

For the LLA16 $\mathrm{EO}_{\mathrm{n}}$ copolymers which preferentially self-assemble into a spherical morphology, the hydrodynamic radius $R_{\mathrm{H}}$, the size of the lyophobic core $R_{\text {core }}$ and the aggregation number $g$ of the resulting micelles have been estimated as reported previously. ${ }^{4}$ It is important to note that in the spherical geometry the aggregation number $g$ indicates the actual number of copolymer molecules composing the assembly, in cylindrical geometry it represents the number of copolymer molecules per unit length, while in flat geometry it corresponds to the number of copolymer molecules per unit area of the bilayer. 
Table S2: Predicted values using the SFC computations

\begin{tabular}{|c|c|c|c|}
\hline BCP & $R_{\mathrm{H}}(\mathrm{nm})^{\mathrm{a}}$ & $R_{\text {core }}(\mathrm{nm})^{\mathrm{b}}$ & $g^{\mathrm{c}}$ \\
\hline LLA8-EO$_{17}$ & 5.4 & 3.2 & 120 \\
\hline $\mathrm{LLA}_{16}-\mathrm{EO}_{30}$ & 8.1 & 5.6 & 228 \\
\hline${\text { LLA } 16-\mathrm{EO}_{34}}$ & 8.3 & 5.6 & 210 \\
\hline $\mathrm{LLA}_{16}-\mathrm{EO}_{38}$ & 8.6 & 5.6 & 194 \\
\hline LLA $_{16}-\mathrm{EO}_{42}$ & 8.8 & 5.6 & 190 \\
\hline $\mathrm{LLA}_{16}-\mathrm{EO}_{46}$ & 9.0 & 5.6 & 185 \\
\hline LLA $_{16}-\mathrm{EO}_{50}$ & 9.2 & 5.6 & 182 \\
\hline
\end{tabular}

a)

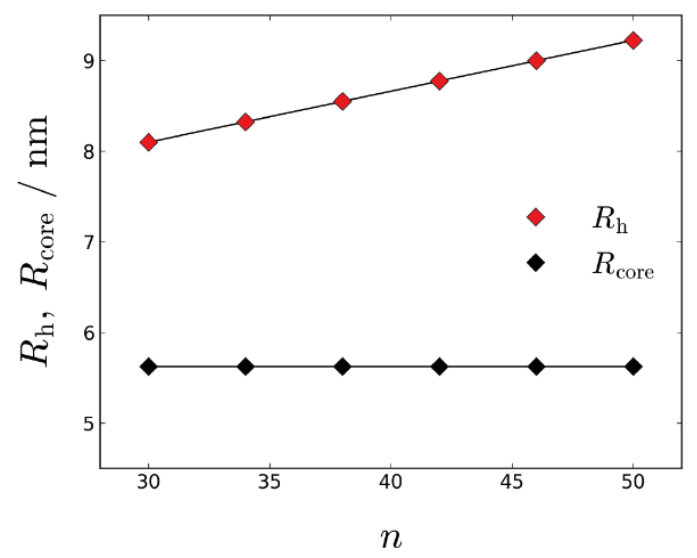

b)

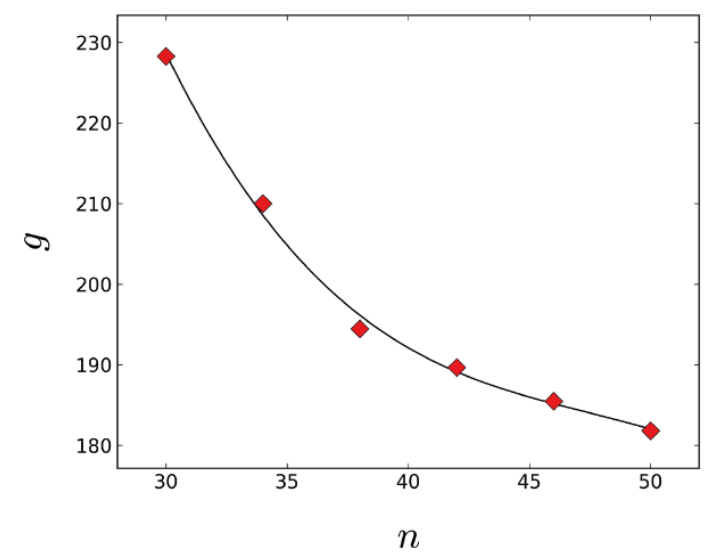

Figure S14. a) PEO block length $(n)$ dependence of the hydrodynamic $\left(R_{H}\right)$ and core $\left(R_{\text {core }}\right)$ radii and b) aggregation number $g$ for spherical micelles of $\mathrm{LA}_{16}-\mathrm{EO}_{\mathrm{n}}$ block copolymers.

\section{Sample preparation}

A widely used preparation procedure for nanoparticles is a precipitation method called solvent switch. ${ }^{8,9}$ This method makes use of a co-solvent and a non-solvent. Firstly, the BCOs are dissolved in the co-solvent. This solvent should be a good solvent for both the hydrophobic and the hydrophilic blocks and should be miscible with water. Examples of frequently used cosolvents are DMF, alcohols, acetone or THF. ${ }^{10}$ Nanoparticles are formed when the non-solvent, water, is added to the BCOs in the co-solvent, or the other way around. Because of the addition of the non-solvent, the homogeneous solution transitions to a solution with precipitated solute, it shifts from a single phase to two phases. ${ }^{11}$ The speed at which solvent switch happens greatly affects the morphology of the self-assembled nanoparticles. There are two extremes, quick injection or gradual phase transition. ${ }^{4}$ For quick injection, the formation of nanoparticles is 
induced by the rapid injection of the BCOs in the co-solvent to the non-solvent. For gradual phase transition, nucleation occurs due to the gradual transition of the system over the phase boundary. This is accomplished by slowly adding the non-solvent water to the co-solvent with BCOs. ${ }^{9}$. The method of quick injection fits best to BCOs which are expected to self-assemble in spherical micelles. Because of the fast solvent shift, the hydrophobic blocks reduce their interfacial area with the solvent very rapidly and as a result spherical micelles are formed. The procedure of gradual phase transition fits best to BCOs which are expected to self-assemble into vesicles as these BCOs need more time to rearrange into their equilibrium structures. When the solvent switch happens here is too fast, unwanted spherical micelles develop as well.4,12.

\section{LLA16EO $_{48}$}

A fast solvent switch should lead to the formation of spherical micelles. The following preparation methods were tested:

A: Quick injection of THF stock solution into vigorously stirring water

B: Dropwise addition of water to the THF stock solution under vigorous stirring

C: Quick addition of water to the THF stock solution under vigorous stirring

For all three conditions particles with a diameter of $20 \mathrm{~nm}$ were observed, however for procedure B and C as well a second population with a diameter of $220 \mathrm{~nm}$ was present as well (Figure S15). These experiments were performed in a glass flat bottom HPLC vial and $30 \mu \mathrm{L}$ of a THF stock solution of $9 \mathrm{mg} / \mathrm{mL}$ were mixed with $270 \mu \mathrm{L}$ of filtered MilliQ water.

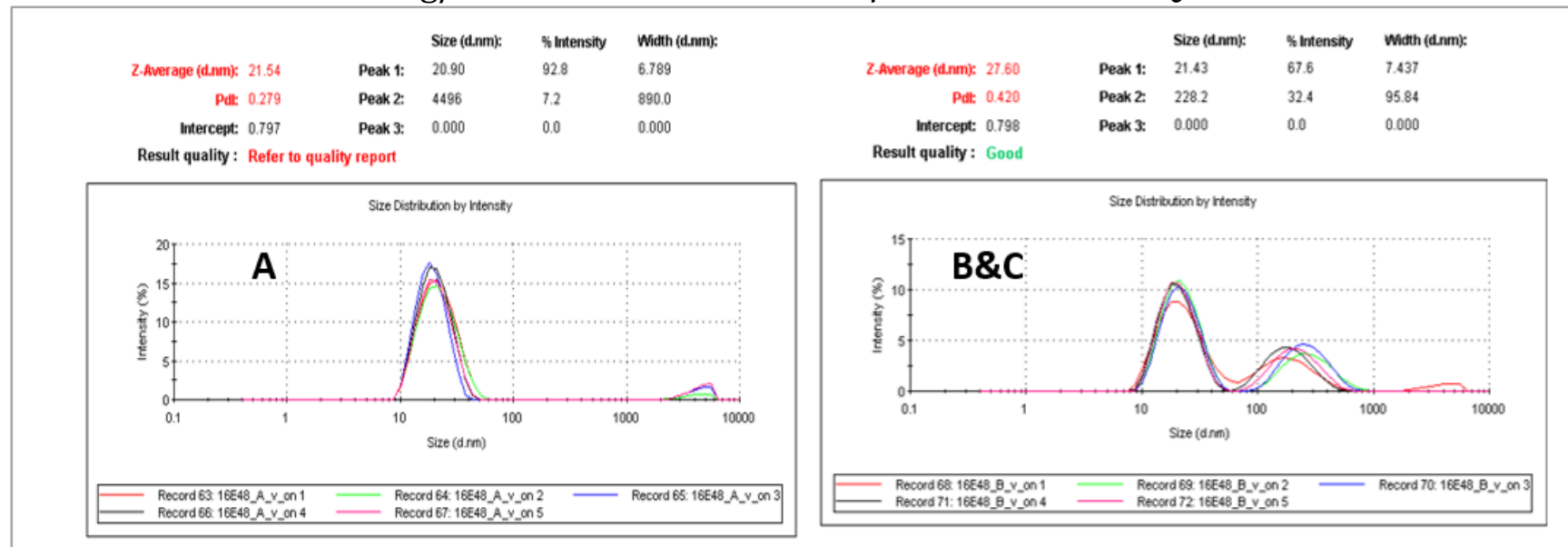

Figure S15: DLS Size distribution by intensity traces of sample preparation A (left) and B\&C (right).

Preparation method A was repeated using different volumes of the THF stock solution leading to different concentration of particles $(0.9 \mathrm{mg} / \mathrm{mL}, 2.2 \mathrm{mg} / \mathrm{mL}, 3.9 \mathrm{mg} / \mathrm{mL})$. To achieve these concentrations different volumes of the $9 \mathrm{mg} / \mathrm{mL}$ stock solution were used $(0.9 \mathrm{mg} / \mathrm{mL}: 30 \mu \mathrm{L}$ stock and $270 \mu \mathrm{L}$ MilliQ; $2.2 \mathrm{mg} / \mathrm{mL}: 60 \mu \mathrm{L}$ stock and $240 \mu \mathrm{L}$ MilliQ; $3.9 \mathrm{mg} / \mathrm{mL}: 90 \mu \mathrm{L}$ stock and $210 \mu \mathrm{L}$ MilliQ). All three concentrations gave the same result (Figure S16): 

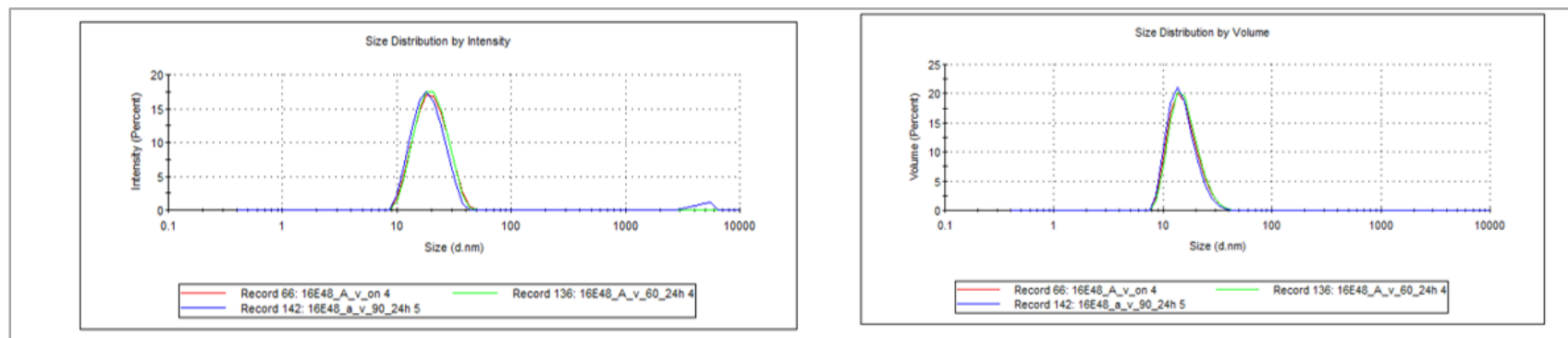

Figure S16: DLS size distribution by intensity (left) and by volume (right) traces of samples of three different concentrations.

Several samples were followed over time with SLS. The higher radius for the first measurement directly after preparation is due to the presence of THF. THF evaporates overtime, which leads to a tighter packing of the core and a drop in $\mathrm{R}_{\mathrm{H}}$. The hydrodynamic radius stays relatively constant overtime and stabilized between 10-12 nm (Figure S17).

\section{Rh of samples over time}

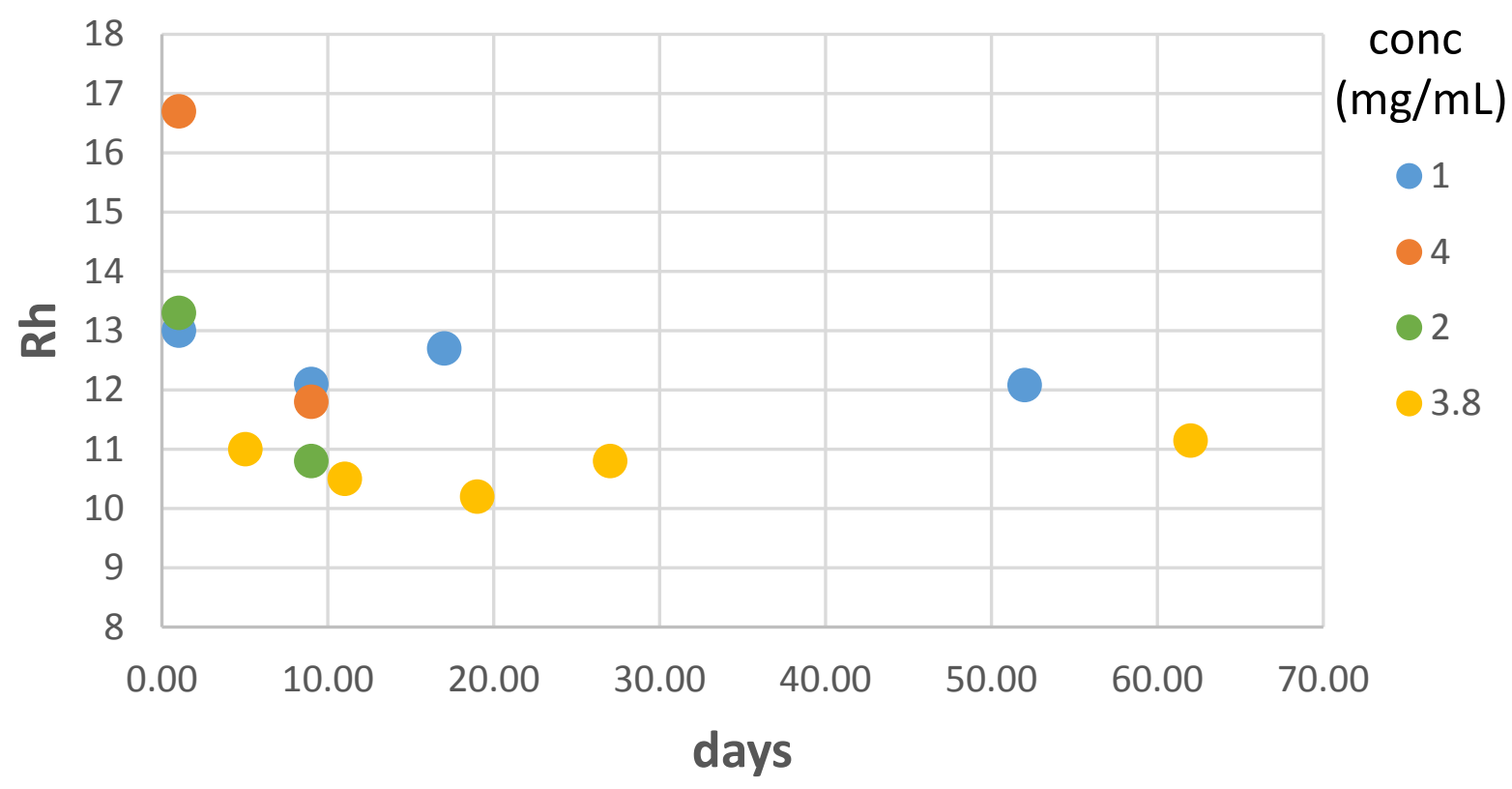

Figure S17: Hydrodynamic radius of samples of $\mathrm{LLA}_{16} \mathrm{EO}_{48}$ of different concentrations overtime.

The samples with the concentration of $1 \mathrm{mg} / \mathrm{mL}$ and $4 \mathrm{mg} / \mathrm{mL}$ were measured with SAXS at the ESRF in Grenoble. 

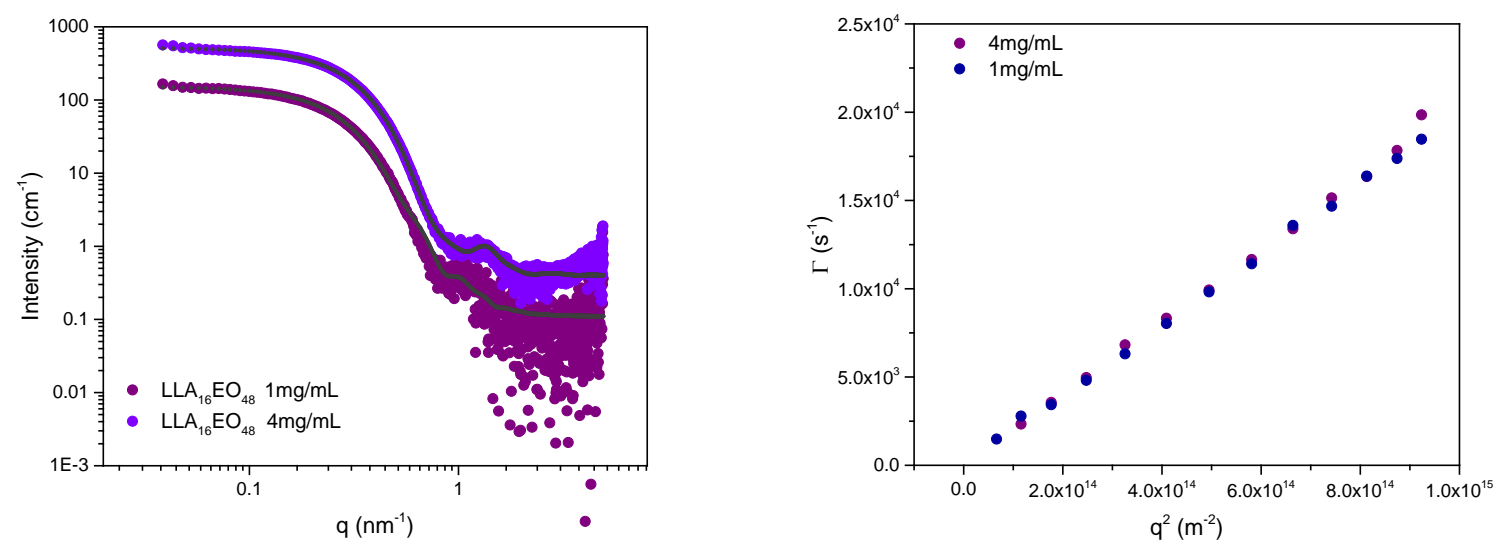

Figure S18: SAXS (left) and DLS (right) of LLA ${ }_{16} \mathrm{EO}_{48}$ at 4 and $1 \mathrm{mg} / \mathrm{mL}$. SAXS scattering data was fitted with a core-shell sphere model (see below) with a radius of $4.2 \mathrm{~nm}$ and a shell of $4.2 \mathrm{~nm}$ for $4 \mathrm{mg} / \mathrm{mL}$ and a radius of $4.7 \mathrm{~nm}$ and a shell of $5 \mathrm{~nm}$ for $1 \mathrm{mg} / \mathrm{mL}$. 


\section{$\mathrm{LLABEO}_{17}$}

The same three preparation methods as for $\mathrm{LLA}_{16} \mathrm{EO}_{48}$ were tested for $\mathrm{LLA}_{8} \mathrm{EO}_{17}$ with the same outcome. Sample preparation A (quick injection into vigorously stirring water) gave a single population, here with a diameter of $12 \mathrm{~nm}$.

\section{Size Distribution by Intensity}

a)

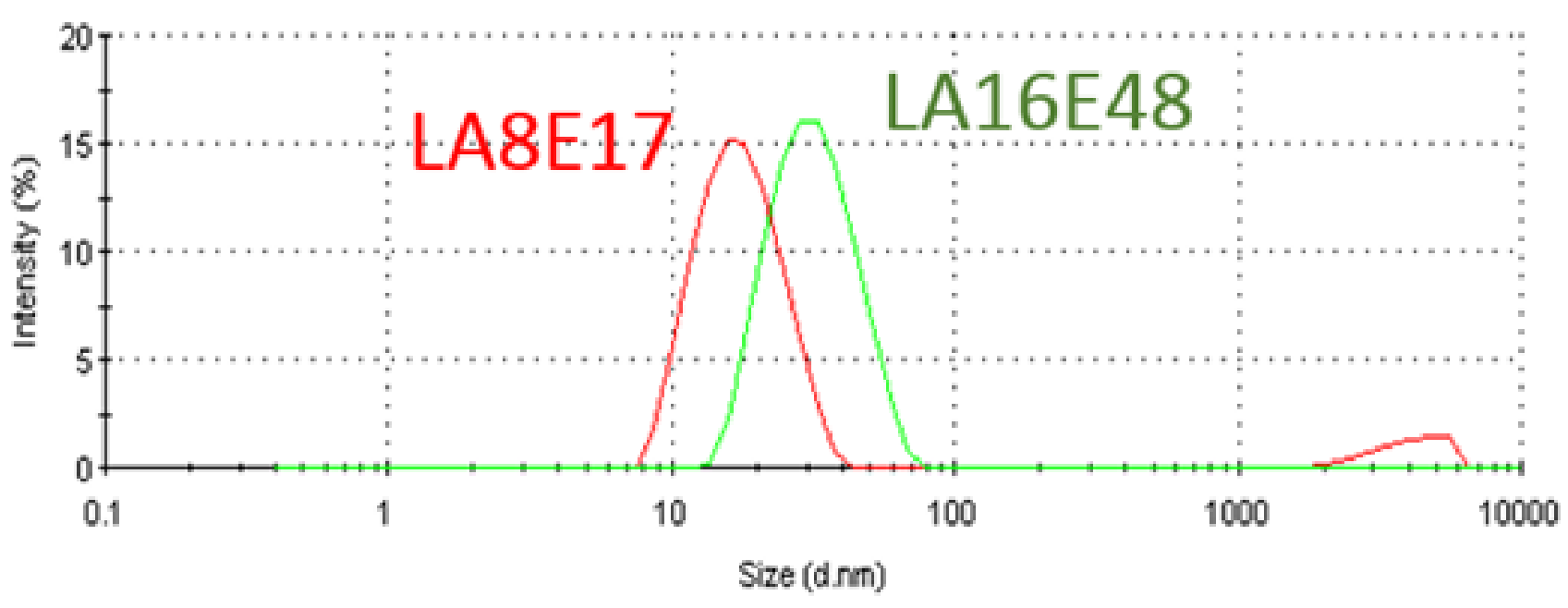

b)

Raw Correlotion Data

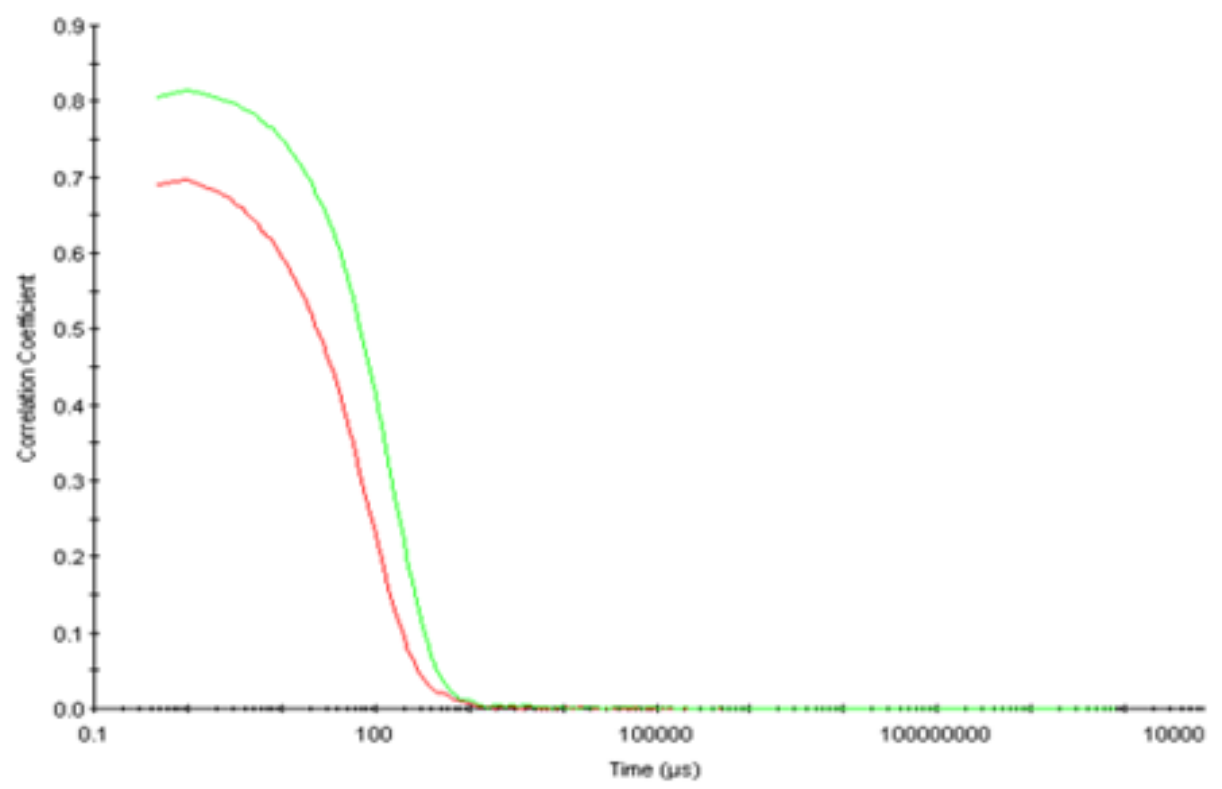

Figure S19: Comparison of $\mathrm{LLA}_{8} \mathrm{EO}_{17}$ and $\mathrm{LLA}_{16} \mathrm{EO}_{48}$ at $4 \mathrm{mg} / \mathrm{mL}$ : (a) overlay of size distributions by intensity and (b) overlay of corresponding correlograms. 
For LLA8EO 17 a sample with the concentration of $4 \mathrm{mg} / \mathrm{mL}$ was analyzed by SAXS:

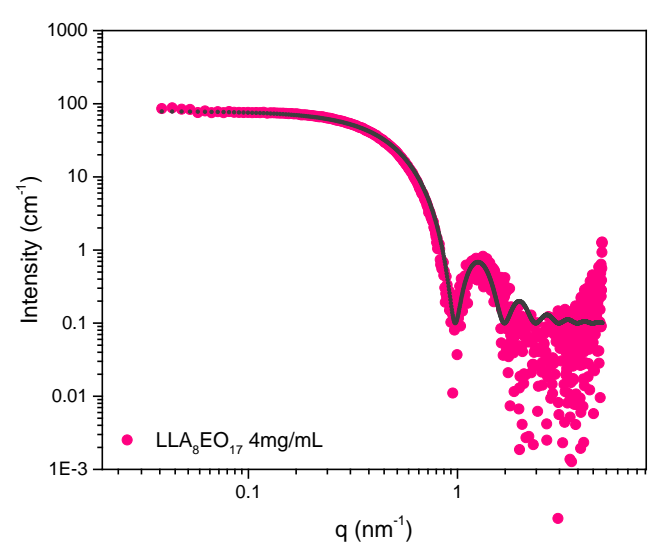

(a)

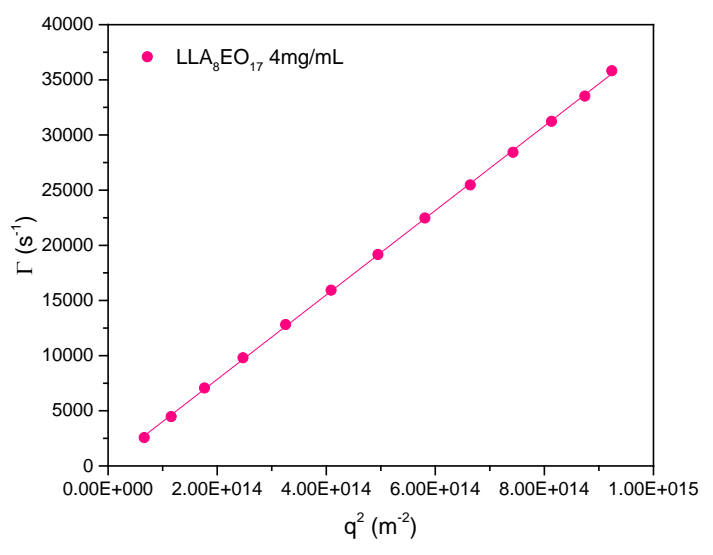

(b)

Figure S20: SAXS (a) and DLS (b) of LLAsE0 17 at $4 \mathrm{mg} / \mathrm{mL}$. SAXS scattering data was fitted with a sphere model (see below) with a radius of $4.6 \mathrm{~nm}$.

\section{LLA16EO $_{11}$}

For this compound the predicted morphology are flat bilyaers/vesicles. Quick injection in THF gave didn't give reproducible results, therefore 2 different approaches of a slow solvent switch were tested:

C: Dropwise addition of water to a THF stock solution in a sonication bath

D: Dropwise addition of water to a stirring THF stock solution using a syringe pump $[0.05$ $\mathrm{mL} / \mathrm{min}$ )

Both sample preparations led to similar results: However, it is crucial that all sample history is erased when dissolving the BCO in THF. Over time, the BCO in the bulk crystallises and it becomes very difficult to completely erase all crystallites during the dissolution. Therefore it is important that the BCO is stirred for xxx h in THF, followed by freeze drying. After this, a stock solution in THF is prepared and water is added. 


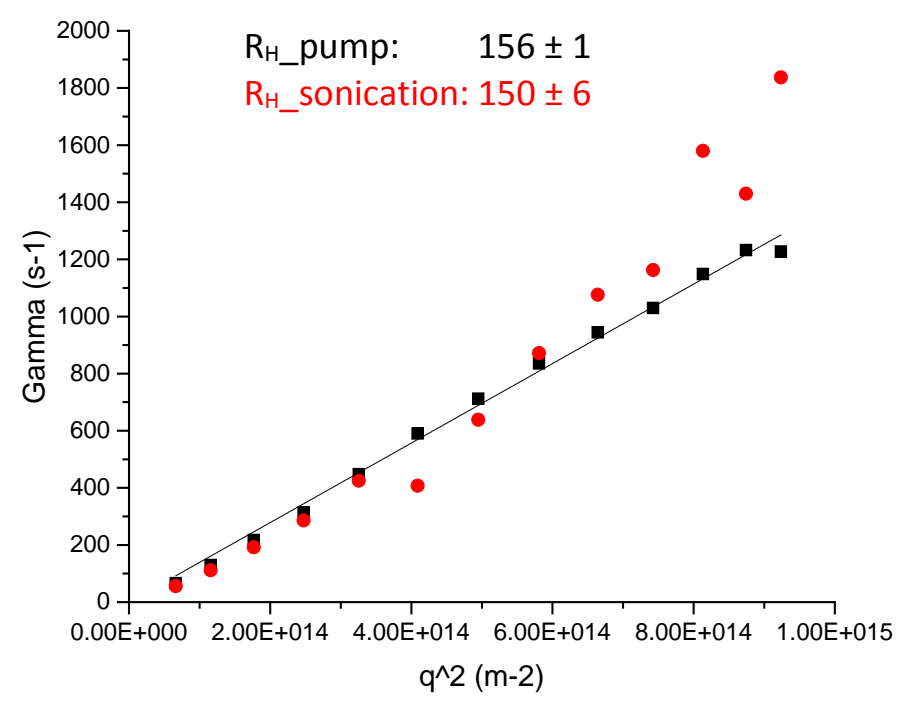

Figure S21: DLS of $\mathrm{LLA}_{16} \mathrm{EO}_{11}$ at prepared by method C (red, $2 \mathrm{mg} / \mathrm{mL}$, sonication) and by method D (black, $1.25 \mathrm{mg} / \mathrm{mL}$, syringe pump).

Vial and stirring bar geometry were varied but did not had an effect on the final particle size (data not shown).

For LLA ${ }_{16} \mathrm{EO}_{11}$ three samples with concentrations of $0.3,1.25$ and $2 \mathrm{mg} / \mathrm{mL}$ were analyzed by SAXS:

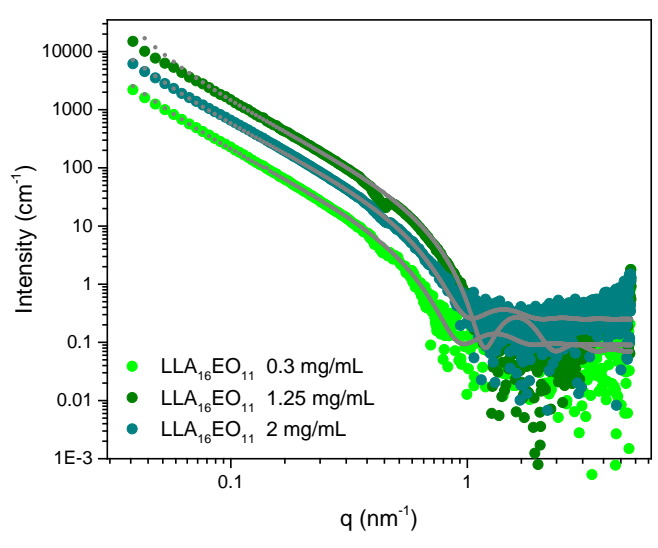

(a)

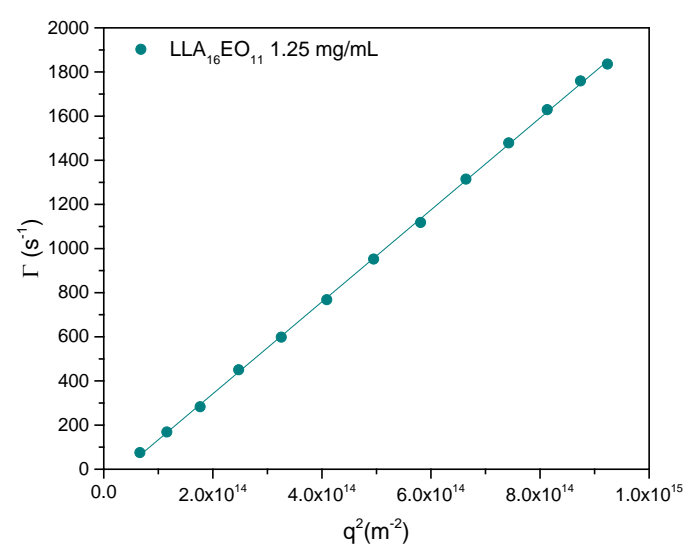

(b)

Figure S22: SAXS (a) of LLA16EO 11 of samples of $0.3 \mathrm{mg} / \mathrm{mL}, 1.25 \mathrm{mg} / \mathrm{mL}$ and $2 \mathrm{mg} / \mathrm{mL}$; DLS (b) of $\mathrm{LLA}_{16} \mathrm{EO}_{11}$ of $1.25 \mathrm{mg} / \mathrm{mL}$. SAXS scattering data was fitted to a lamellar structure with a contribution from a form factor of spheres (see below) with a bilayer thickness of $6 \mathrm{~nm}(1.25$ $\mathrm{mg} / \mathrm{mL}), 6.5 \mathrm{~nm}(0.3 \mathrm{mg} / \mathrm{mL})$, and $5.3 \mathrm{~nm}(2 \mathrm{mg} / \mathrm{mL})$. 


\section{LLA16EO5}

As predicted, morphologies formed by this compound were unstable in water. All preparation methods (slow addition with a syringe pump (D), dropwise addition during sonication (C)) led initially to the formation of vesicles with $\mathrm{R}_{\mathrm{H}}$ of $100 \mathrm{~nm}$, but after overnight the compound precipitated out of the solution (see Figure S23 right). As a result, this compound wasn't investigated further.

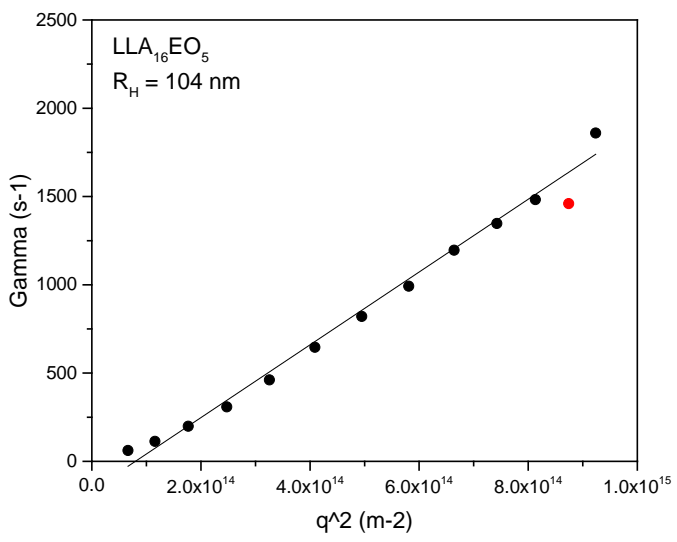

(a)

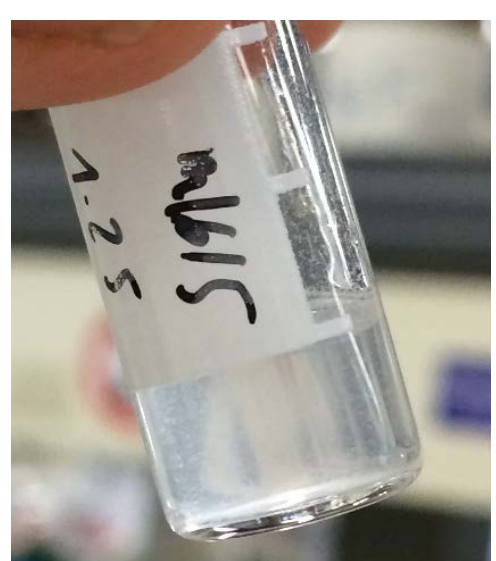

(b)

Figure S23: (a) DLS of LLA16EO5 at $1.25 \mathrm{mg} / \mathrm{mL}$; (b) image taken of a LLA16EO5 solution after overnight at RT.

\section{LA16EO17 $_{17}$}

For this compound the self-assembly into cylindrical micelles was predicted. Quick injection in this case didn't give reproducible results, therefore 2 different approaches of a slow solvent switch were tested:

C: Dropwise addition of water to a THF stock solution in a sonication bath

D: Dropwise addition of water to a stirring THF stock solution using a syringe pump $(0.05$ $\mathrm{mL} / \mathrm{min}$ )

Due to their non-spherical morphology samples could not be analysed using DLS. 


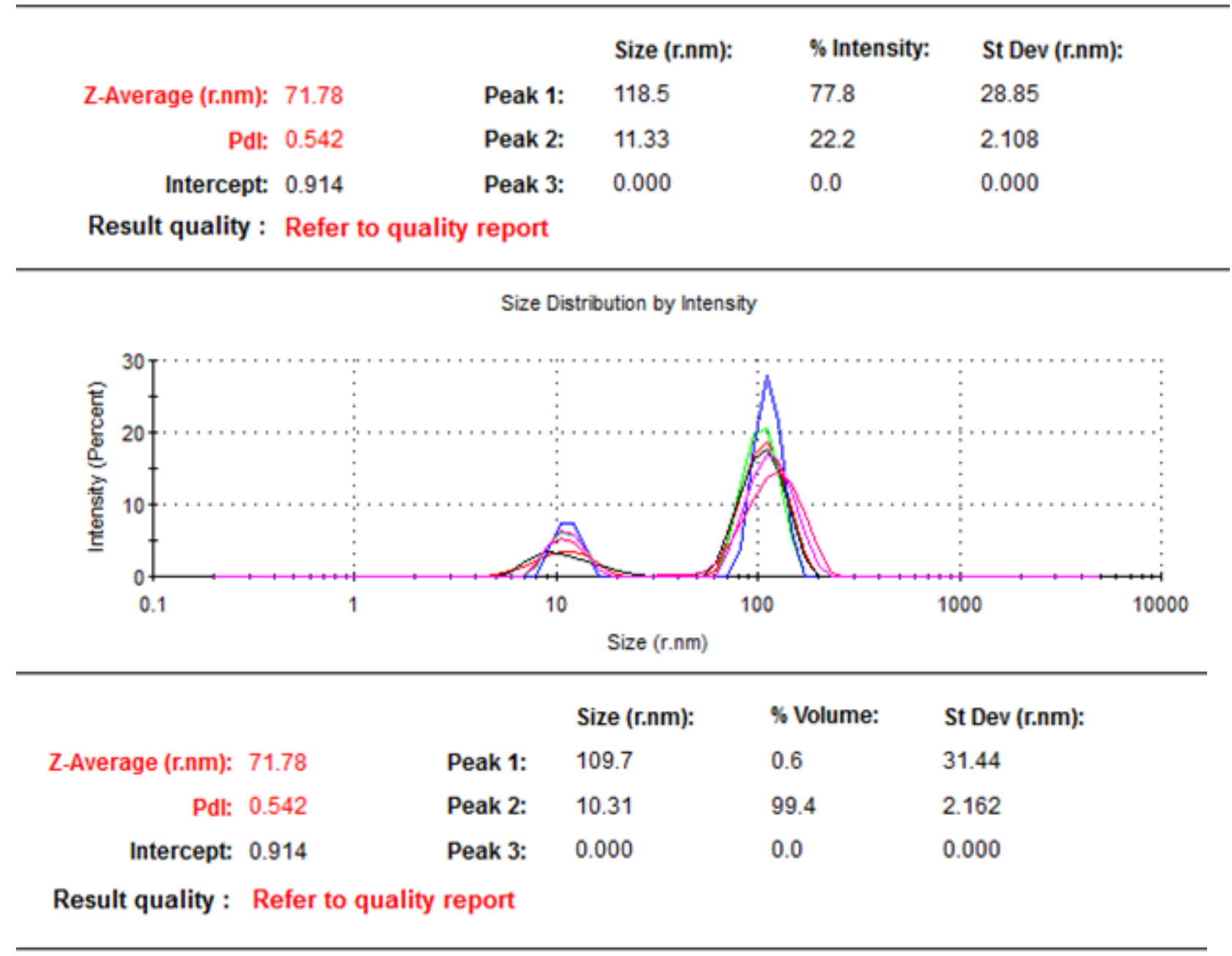

Size Distribution by Volume

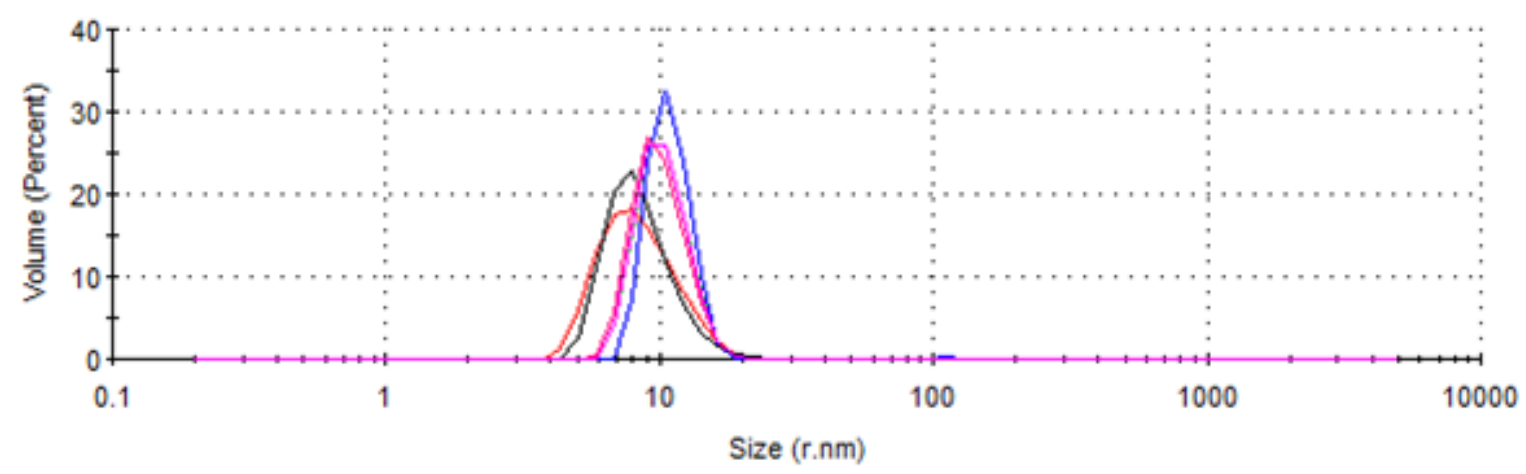

Figure S24: Size distribution by intensity (top) and by volume (bottom) of $1 \mathrm{mg} / \mathrm{mL}$ sample of $\mathrm{LLA}_{16} \mathrm{EO}_{17}$.

Samples prepared with method $\mathrm{C}$ gave slightly more reproducible results, with a fractional dimension $\left(\log \left(I_{S} \sin (\theta)\right)\right.$ vs $\left.\log (q)\right)$ much closer to 1 . 


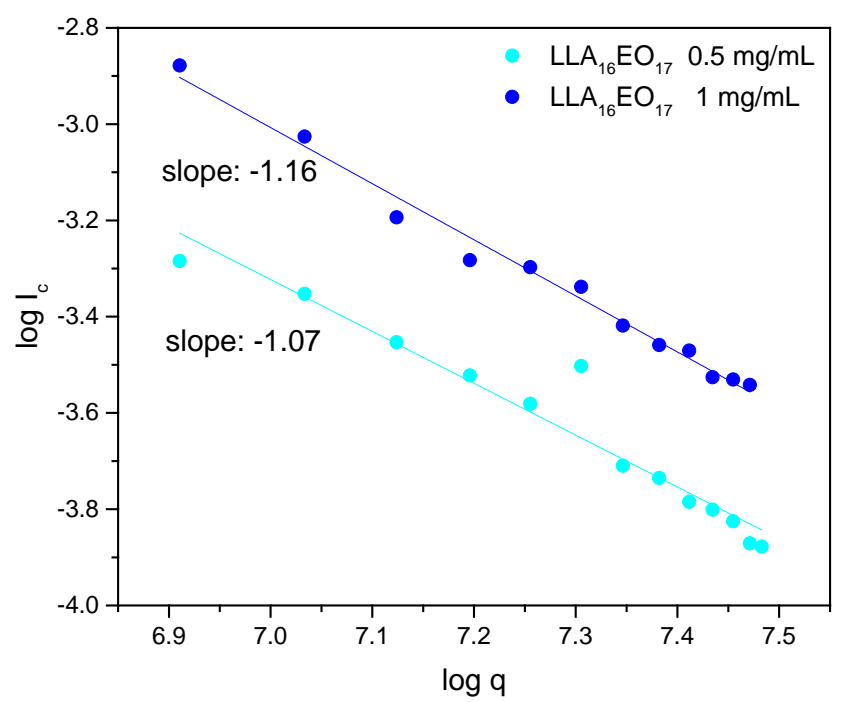

Figure S25: Fractal dimensions of $1 \mathrm{mg} / \mathrm{mL}$ (blue) and $0.5 \mathrm{mg} / \mathrm{mL}$ (cyan) sample of LLA16 $\mathrm{EO}_{17}$.

For $\mathrm{LLA}_{16} \mathrm{EO}_{17}$ three samples with concentrations of $0.5,1$ and $2.5 \mathrm{mg} / \mathrm{mL}$ were analyzed by SAXS (for all concentrations see Figure S27):

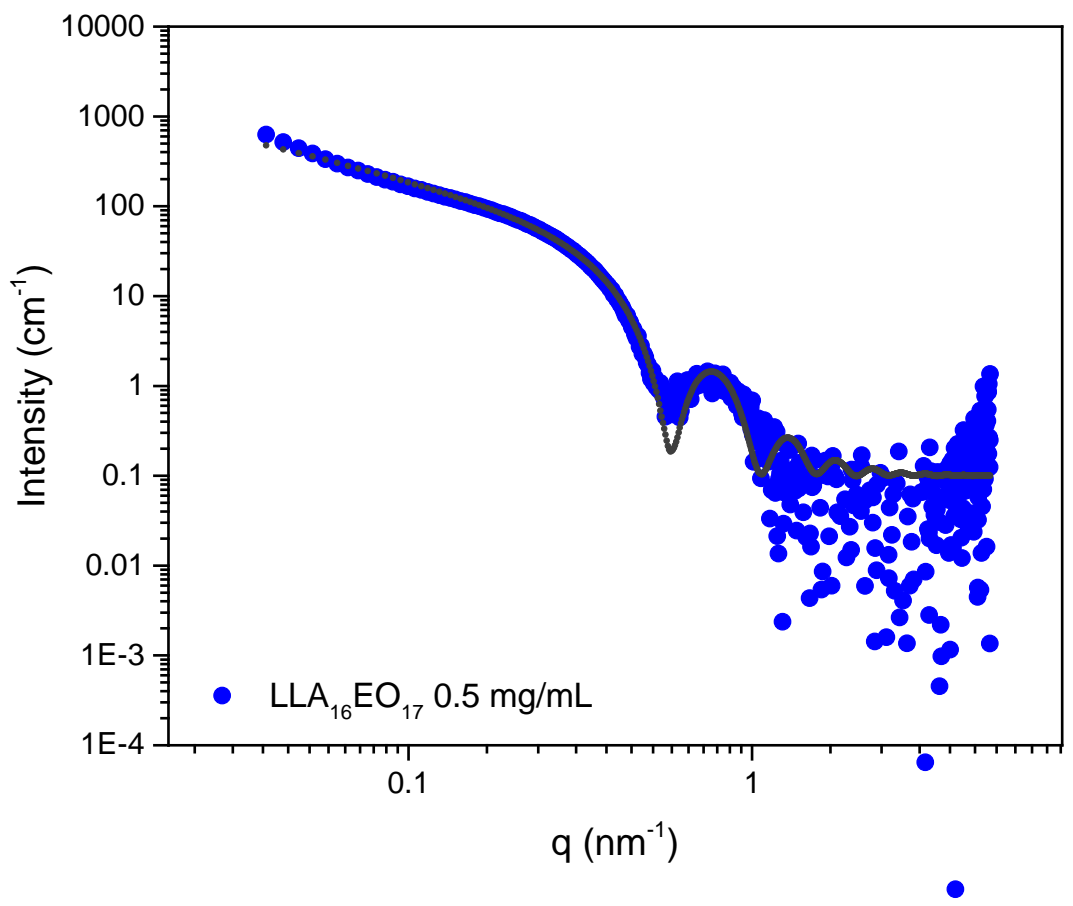

Figure S26: SAXS profile of $0.5 \mathrm{mg} / \mathrm{mL}$ sample of LLA16EO17. SAXS scattering data was fitted to a cylinder model (see below) with a cross-sectional radius of $6.6 \mathrm{~nm}$. 


\section{SAXS data analysis}

The scattering intensity $I(q)$ is given by the product of the form factor, $P(q)$, and the structure factor $S$ (q) plus incoherent scattering contributions (background):

$$
I(q)=N(\Delta \rho)^{2} * P(q) S(q)+\text { background }
$$

Where $N$ is the number density of scattering objects, and $\Delta \rho$ is the contrast term of the electronic density between the objects and the solvent. At the measured concentrations, the scattering profile is mainly determined by $P(q)$. Except for $\mathrm{LLA}_{16} \mathrm{EO}_{17}$ which presents concentration dependent scattering curves (Figure S27). Fittings were performed using SasView ® software. The electron density of water was fixed to 0.33 electrons $/ \AA^{3}$ and the electron density of the polymer was let to float during the fitting.

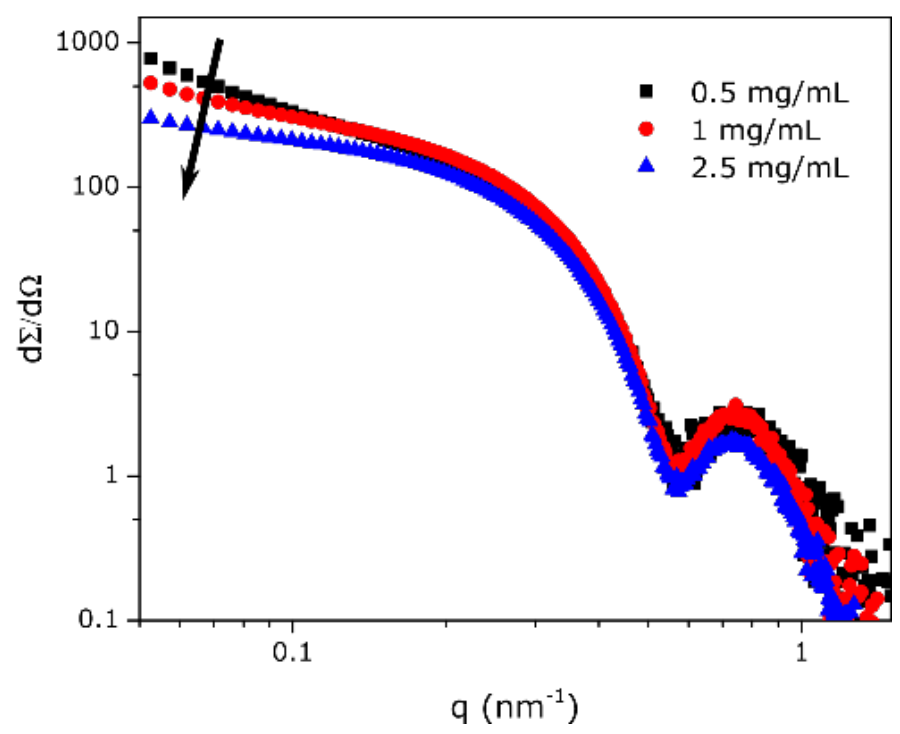

Figure S27: Concentration normalized scattering curves of $\mathrm{LLA}_{16} \mathrm{EO}_{17}$.

Scattering spectra obtained from $\mathrm{LLA}_{8} \mathrm{EO}_{17}$ samples can be described by the sphere model:

$$
I(q)=\frac{\text { scale }}{V}\left[3 V(\Delta \rho) \frac{\sin (q r)-q r \cos (q r)}{(q r)^{3}}\right]^{2}
$$

Where $V$ is the volume of the scatterer, $r$, is the radius of the sphere, $\Delta \rho$ is the contrast factor and scale is a volume fraction. Within this model spheres are considered with a homogeneous density distribution within the particle. Scattering curves from $\mathrm{LLA}_{16} \mathrm{EO}_{48}$ are described as core-shell spheres:

$$
\begin{array}{r}
I(q)=\frac{\text { scale }}{V_{2}} * \frac{3}{V_{2}}\left[V_{1}\left(\rho_{1}-\rho_{2}\right) \frac{\sin \left(q r_{1}\right)-q r \cos \left(q r_{1}\right)}{\left(q r_{1}\right)^{3}}\right. \\
\left.+V_{2}\left(\rho_{2}-\rho_{s}\right) \frac{\sin \left(q r_{2}\right)-q r \cos \left(q r_{2}\right)}{\left(q r_{2}\right)^{3}}\right]
\end{array}
$$


Where $V_{2}$ and $V_{1}$ are the volume of the whole particle and core respectively, $r_{2}=r_{1}+$ shell thickness is the radius of the particle and $r_{1}$ is the radius of the core. The model accounts for a difference in electronic density between the core (LLA) and the shell (EO). This is a fair assumption considering the longer EO chain present, which allows to differentiate between core and shell. A small polydispersity (c.a. 0.1) in the size of the micelles is introduced by averaging the form factor over a Schultz distribution.

LLA ${ }_{16} \mathrm{E}_{11}$ curves were fitted to a lamellar structure with uniform electron density and random distribution. This model fits well at mid and high $q$ values but deviates at low $q$. According to TIRF microscopy (Figure S13) and DLS measurements (Figure S21) particles of. $>100 \mathrm{~nm}$ in radius were present. In order to include these particles, we also added contributions from a form factor of spheres of sizes $>100 \mathrm{~nm}$ (Eq. 5). Again, polydispersity in the size of the spheres is introduced by averaging the form factor over a Schultz distribution.

$$
I(q)=N_{l} * \frac{2 \pi}{q^{2} \delta}\left(\frac{2 \Delta \rho_{l}^{2}}{q^{2}}(1-\cos (q \delta))+N_{h s}\left[3 V\left(\Delta \rho_{r}\right) \frac{\sin (q r)-q r \cos (q r)}{(q r)^{3}}\right]^{2}\right.
$$

Where $N_{l}, N_{h s}$ and $\delta$ are the number density of lamellar, hard spheres and bilayer thickness respectively.

Scattering patterns of $\mathrm{LLA}_{16} \mathrm{E}_{17}$ structures were fitted to a form factor of randomly oriented cylinders (Eq. 6). The model fits well at high and mid $q$, however it deviates at low $q$ due to interparticle interactions. To account for this effect we included a hard - sphere structure factor with an effective radius of c.a. $4 \mathrm{~nm}$.

$$
I(q)=\frac{\text { scale }}{V} \int_{0}^{\pi / 2}\left[2(\Delta \rho) V \frac{\sin \left(\frac{1}{2} q L \cos (\alpha)\right) * J_{1}(q R \sin (\alpha)}{\frac{1}{2} q L \cos (\alpha) * q R \sin (\alpha)}\right]^{2} \sin (\alpha) d \alpha
$$

Where $\alpha, L, R$ are the angle between the axis of the cylinder and $\vec{q}$, the length and radius of the cylinder. $J 1$ is the first order Bessel function. 


\section{References}

${ }^{1}$ van Genabeek, B.; Lamers, B. A. G.; de Waal, B. F. M.; van Son, M. H. C.; Palmans, A. R. A.; Meijer, E. W. Amplifying (Im)Perfection: The Impact of Crystallinity in Discrete and Disperse Block CoOligomers. J. Am. Chem. Soc., 2017, 139, 14869-14872.

${ }^{2}$ Lamers,B.A.G. van Genabeek, B.; Hennissen,J.; de Waal, B.F.M.; Palmans, A.R.A.; Meijer, E.W. Stereocomplexes of Discrete, Isotactic Lactic Acid Oligomers Conjugated with Oligodimethylsiloxanes. Macromolecules, 2019, 52, 1200-1209.

${ }^{3}$ Kripotou, S.; Psylla, C.; Kyriakos, K.; Raftopoulos, K. N.; Zhao, J.; Zhang, G.; Pispas, S.; Papadakis, C. M.; Kyritsis, A. Structure and Crystallization Behavior of Poly(Ethylene Oxide) (PEO) Chains in Core-Shell Brush Copolymers with Poly(Propylene Oxide)-Block-Poly(Ethylene Oxide) Side Chains. Macromolecules, 2016, 49, 5963-5977.

4 Ozdemir, C.; Gunter, A. Solution Thermodynamics of Poly(Ethylene Glycol)/Water Systems. J. Appl. Polym. Sci., 2006, 101, 203-216.

5 Sharp, J. S.; Forrest, J. A.; Jones, R. A. L. Swelling of Poly(DL-Lactide) and Polylactide-Co-Glycolide in Humid Environments. Macromolecules, 2001, 34, 8752-8760.

${ }^{6}$ Ianiro, A.; Patterson, J.; Rijt, M. M. J. Van; Hendrix, M. M. R. M.; Sommerdijk, N. A. J. M.; Voets, I. K.; Esteves, A. C. C.; Tuinier, R. A Roadmap for Poly(Ethylene Oxide)-Block-Poly- $\varepsilon$-Caprolactone SelfAssembly in Water : Prediction, Synthesis , and Characterization. J. Polym. Sci. Part B-Polym. Phys., 2018, 56, 330-339.

7 Butt, H.-J.; Graf, K.; Kappl, M. Physics and Chemistry of Interfaces; WILEY-VCH Verlag GmbH \& Co. KGaA, 2006.

8 Texter, J. Precipitation and Condensation of Organic Particles. J. Dispers. Sci. Technol., 2001, 22, 499-527.

9 Brick, M. C.; Palmer, H. J.; Whitesides, T. H. Formation of Colloidal Dispersions of Organic Materials in Aqueous Media by Solvent Shifting. Langmuir, 2003, 19, 6367-6380.

10 Mai, Y.; Eisenberg, A. Self-Assembly of Block Copolymers. Chem. Soc. Rev., 2012, 41, 5969-5985. 11 Van Keuren, E. R. Polymer Nanoparticles Synthesized with Solvent Shifting. J. Dispers. Sci. Technol., 2005, 25, 547-553.

12 Konishcheva, E. V.; Zhumaev, U. E.; Meier, W. P. PEO- $b$-PCL- $b$-PMOXA Triblock Copolymers: From Synthesis to Microscale Polymersomes with Asymmetric Membrane. Macromolecules 2017, $50,1512-1520$ 